\title{
The Asymptotic Behavior of Singular Solutions of Some Nonlinear Partial Differential Equations in the Complex Domain
}

\author{
By \\ Sunao $\overline{\mathrm{OUCHI}}{ }^{*}$
}

\begin{abstract}
Let $u(t, x)\left((t, x) \in \mathbb{C} \times \mathbb{C}^{d}\right)$ be a solution of a nonlinear partial differential equation in a neighborhood of the origin, which is not necessarily holomorphic on $\{t=0\}$. We study the asymptotic behavior of $u(t, x)$ as $t \rightarrow 0$ and give its asymptotic terms with remainder estimate of Gevrey type.
\end{abstract}

\section{$\S 0 . \quad$ Introduction}

Let $L(u)=0$ be a nonlinear partial differential equation in a neighborhood of the origin of $\mathbf{C}^{d+1}$ and $K$ be a complex hypersurface through the origin. We choose a coordinate so that $K=\{t=0\}$. Other coordinates are written by $x \in \mathbf{C}^{d}$, so $(t, x)=\left(t, x_{1}, x_{2}, \cdots, x_{d}\right) \in \mathbf{C}^{d+1}$. Suppose that $u(t, x)$ solves $L(u)=0$, which is not necessarily holomorphic on $K$. The aims of this paper are to study the behavior of a singular solution $u(t, x)$ near $K$ and to obtain asymptotic expansion of $u(t, x)$ as $t$ tends to 0 more concretely.

The behaviors of singular solutions of linear equations were studied in [5], [6], [7] and [8]. We summarize some of results of these papers. Let $L\left(t, x, \partial_{t}, \partial_{x}\right)$ be a linear partial differential operator with holomorphic coefficients. Consider

Communicated by T. Kawai. Received November 27, 2006. Revised July 20, 2007, October 24, 2007.

2000 Mathematics Subject Classification(s): 35A20, 35B40, 35C20.

Key words: singular solution, asymptotic behavior, complex partial differential equation, Mellin transform.

*Department of Mathematics, Sophia University, Tokyo 102-8554, Japan.

e-mail: ouchi@mm.sophia.ac.jp

(C) 2008 Research Institute for Mathematical Sciences, Kyoto University. All rights reserved. 
$L\left(t, x, \partial_{t}, \partial_{x}\right) u=f(t, x)$, where we assume $f(t, x)$ is holomorphic for simplicity. An index $\gamma>0$ is defined for $L\left(t, x, \partial_{t}, \partial_{x}\right)$. Suppose that $u(t, x)$ grows at most infra exponential order $\gamma$, that is, for any $\varepsilon>0$

$$
|u(t, x)| \leq C_{\varepsilon} \exp \left(\varepsilon|t|^{-\gamma}\right) .
$$

The main result in [5] is the following. If $L\left(t, x, \partial_{t}, \partial_{x}\right)$ belongs to some class of operators, then $u(t, x)$ has an asymptotic expansion of power series with remainder with Gevrey type estimate, that is,

$$
\left|u(t, x)-\sum_{n=0}^{N-1} u_{n}(x) t^{n}\right| \leq A B^{N} \Gamma\left(\frac{N}{\gamma}+1\right)|t|^{N} .
$$

This result was generalized in [7]. The analysis of singular solutions of linear equations belonging to a larger class than [5] and [7] was further studied in [6] and [8]. It is shown in [6] for some class of eqations that if a solution $u(t, x)$ satisfies (0.1), then there exist constants $C$ and $c$ such that

$$
|u(t, x)| \leq C|t|^{c}
$$

in a neighborhood of the origin, which means that the growth property of singularities is improved, and its behavior near $K$ was studied in [8].

This paper is a continuation of [8] and treats nonlinear equations and the main aim is a generalization of the results in [8] to nonlinear case. The main results were announced in [10] without proofs. In Section 1 notations, definitions and Mellin type integral are introduced. The conditions on nonlinear partial differential equations to be studied are given and an index $\gamma$ is defined, which depends on solutions. The main results are Theorems 1.3 and 1.5. One of them is the following.

Suppose that $u(t, x)$ with singularities on $K$ solves $L(u)=0$ with $|u(t, x)| \leq$ $C|t|^{\nu_{0}}$ for a constant $\nu_{0}>0$. Then we find asymptotic terms of $u(t, x)$ as $t$ tends to 0 ,

$$
\left\{\begin{aligned}
u(t, x) & \sim \sum_{n=0}^{\infty} u_{n}(t, x) \quad t \rightarrow 0 \\
u_{n}(t, x) & =O\left(|t|^{p_{n}+\nu_{0}}\right) \\
0=p_{0} & <p_{1}<\cdots<p_{n}<\rightarrow+\infty
\end{aligned}\right.
$$

where $\left\{u_{n}(t, x)\right\}_{n \in \mathbb{N}}$ are functions represented by Mellin type integral

$$
u_{n}(t, x)=\frac{1}{2 \pi i} \int_{\mathcal{C}} t^{-\lambda} \frac{\psi_{n}(\lambda, x)}{\varphi_{n}(\lambda, x)} d \lambda .
$$


We also obtain an estimate of the remainder of Gevrey type,

$$
\left|u(t, x)-\sum_{n=0}^{N-1} u_{n}(t, x)\right| \leq A B^{N} \Gamma\left(\frac{p_{N}}{\gamma}+1\right)|t|^{p_{N}+\nu_{0}} .
$$

In Section 2 we sum up majorant functions. Majorant functions are fully used to obtain estimates of holomorphic functions. In particular we have to estimate functions on a sectorial region. Many Propositions and Lemmas are stated without proofs. In Section 3 we study the Mellin transform with respect to $t$ of a solution $u(t, x)$, which is denoted by $(\mathcal{M} u)(\lambda, x)$. The main purpose of Section 3 is to show Theorem 1.3, that is, to show $(\mathcal{M} u)(\lambda, x)$ is a meromorphic function of $\lambda$ in $\mathbb{C}$. In Sections $4-6$ we show Theorem 1.5, which is the other main result. In Section 4 we modify the original equation $L(u)=0$ for our purpose. The coefficients of the obtained equation are not necessarily holomorphic on $K$. In Section 5 we find functions $\left\{u_{n}(t, x)\right\}_{n=0}^{\infty}$ in $(0.4)$ and in Section 6 we show an estimate of the remainder. In Section 7 we give the proofs of propositions and lemmas in Section 2 concerning majorant functions.

\section{§1. Notations, Definitions and Main Results}

Let us introduce notations. $\mathbb{N}=\{0,1,2, \cdots\}$ is the set of all nonnegative integers. $(t, x)=\left(t, x_{1}, \cdots, x_{d}\right) \in \mathbb{C} \times \mathbb{C}^{d},|x|=\max _{1 \leq i \leq d}\left|x_{i}\right|, \alpha=\left(\alpha_{0}, \alpha^{\prime}\right)=$ $\left(\alpha_{0}, \alpha_{1}, \cdots, \alpha_{d}\right) \in \mathbb{N}^{d+1}$ is a multi-index and $|\alpha|=\alpha_{0}+\left|\alpha^{\prime}\right|=\sum_{i=0}^{d} \alpha_{i}$. As for differentiations $\partial_{x_{i}}=\frac{\partial}{\partial x_{i}}, \vartheta=t \frac{\partial}{\partial t}$ and $\vartheta^{\alpha_{0}} \partial^{\alpha^{\prime}}=\left(t \partial_{t}\right)^{\alpha_{0}} \partial_{x_{1}}^{\alpha_{1}} \cdots \partial_{x_{d}}^{\alpha_{d}}$ for a multi-index $\alpha \in \mathbb{N}^{d+1}$. In this paper we study solutions which are holomorphic on a sectorial region. So let us introduce notations about sectorial regions. $U=U_{0} \times U^{\prime}$ is a polydisk with center $(t, x)=(0,0)$, where $U_{0}$ is an open disk in $\mathbb{C}$ and $U^{\prime}=\left\{x ;|x|<R^{\prime}\right\}$. Let $\widehat{U_{0}-\{0\}}$ be the universal covering space of $U_{0}-\{0\} . U_{0}(\theta)=\{t \in \widehat{U-\{0\}} ;|\arg t|<\theta\}$ is a sector and set $U(\theta)=U_{0}(\theta) \times U^{\prime}$, which is sectorial with respect to $t$.

For open sets $V$ and $W, V \Subset W$ means $\bar{V}$ is compact and $\bar{V} \subset W$. However for sectors $S_{0}=\left\{t ; 0<|t|<t_{0},|\arg t|<\theta_{0}\right\}$ and $S_{1}=\left\{t ; 0<|t|<t_{1},|\arg t|<\right.$ $\left.\theta_{1}\right\}, S_{0} \Subset S_{1}$ means $t_{0}<t_{1}$ and $\theta_{0}<\theta_{1} \cdot \mathscr{O}(W)$ is the set of all holomorphic functions on a region $W$ and $\mathscr{O}(W)[\lambda]$ is the set of all polynomials in $\lambda$ with coefficients in $\mathscr{O}(W)$. Solutions considered in this paper are in $\mathscr{O}(U(\theta))$ for a polydisk $U$ and a $\theta>0$.

As stated in introduction the aim of this paper is to study behaviors of singular solutions of nonlinear partial differential equations. Let us introduce 
notations concering nonlinear equations, which are not necessarily usual. Let $\Delta(m)=\left\{\alpha=\left(\alpha_{0}, \alpha^{\prime}\right) \in \mathbb{N} \times \mathbb{N}^{d} ;|\alpha| \leq m\right\}$ and $M=\# \Delta(m)$.

Let $A=\left(A_{\alpha}\right)_{\alpha \in \Delta(m)} \in \mathbb{N}^{M}$. Then $|A|=\sum_{\alpha \in \Delta(m)} A_{\alpha}$. For $A=\left(A_{\alpha}\right), B=$ $\left(B_{\alpha}\right) \in \mathbb{N}^{M} A \leq B$ means $A_{\alpha} \leq B_{\alpha}$ for all $\alpha \in \Delta(m)$. For $Z=\left(Z_{\alpha}\right)_{\alpha \in \Delta(m)} \in$ $\mathbb{C}^{M}$ we set $Z^{A}=\prod_{\alpha \in \Delta(m)} Z_{\alpha}^{A_{\alpha}}$ and if $|A|>0$, there exist $\{\alpha(i) \in \Delta(m) ; 1 \leq$ $i \leq|A|\}$ such that $Z^{A}=\prod_{i=1}^{|A|} Z_{\alpha(i)}$, which we'll often use in the later. Set $\mathbb{N}^{M *}:=\mathbb{N}^{M}-\{0\}=\left\{A \in \mathbb{N}^{M} ;|A|>0\right\}, \mathbb{N}_{\text {lin }}^{M *}=\left\{A \in \mathbb{N}^{M *} ;|A|=1\right\}$ and

$$
\mathbb{N}_{\text {lin }, 0}^{M *}=\left\{A \in \mathbb{N}_{\text {lin }}^{M *} ; A_{\alpha}=1 \text { for some } \alpha=\left(\alpha_{0}, 0\right) \in \mathbb{N} \times \mathbb{N}^{d}\right\} .
$$

For $A=\left(A_{\alpha}\right)_{\alpha \in \Delta(m)} \in \mathbb{N}^{M *}$

$$
m_{A}=\max \left\{|\alpha| ; A_{\alpha} \neq 0\right\} .
$$

Let $U^{\prime}\left(\right.$ resp. $\left.U_{0}\right)$ be a polydisk in $\mathbb{C}^{d}($ resp. $\mathbb{C})$ with center $x=0$ (resp. $t=0)$ and $L(u)$ be a nonlinear partial differential operator with order $m$ in the form

$$
L(u):=L\left(t, x, \vartheta^{\alpha_{0}} \partial^{\alpha^{\prime}} u ;\left(\alpha_{0}, \alpha^{\prime}\right) \in \Delta(m)\right),
$$

where $L(t, x, Z) \in \mathscr{O}\left(U_{0} \times U^{\prime} \times \Omega\right), Z=\left(Z_{\alpha} ; \alpha \in \Delta(m)\right)$ and $\Omega$ being a neighborhood of $Z=0$ in $\mathbb{C}^{M}$. We can decompose $L(u)$ as follows:

$$
L(u)=P u+Q(u)+f(t, x),
$$

where

$$
\left\{\begin{aligned}
P u & =\sum_{h=0}^{m} c_{h}(t, x) \vartheta^{h} u(t, x), \\
Q(u) & =\sum_{A \in \mathbb{N}^{M *}-\mathbb{N}_{l i n, 0}^{M *}} c_{A}(t, x) \prod_{\alpha \in \Delta(m)}\left(\vartheta^{\alpha_{0}} \partial^{\alpha^{\prime}} u(t, x)\right)^{A_{\alpha}}, \\
f(t, x) & =L(t, x, 0) .
\end{aligned}\right.
$$

Here $c_{h}(t, x), c_{A}(t, x)$ and $f(t, x)$ are holomorphic in $U_{0} \times U^{\prime}$. We assume there exists $k \in\{0,1, \cdots, m\}$ such that

$$
c_{k}(0, x) \neq 0 \text { for } x \in U^{\prime} \text { and } c_{h}(0, x)=0 \text { for } h>k
$$

and

$$
c_{A}(0, x)=0 \quad \text { for } \quad A \in \mathbb{N}_{l i n}^{M *}-\mathbb{N}_{l i n, 0}^{M *} .
$$


Hence $\sum_{h=0}^{k} c_{h}(t, x) \vartheta^{h}$ is a $k$-th order ordinary differential operator with regular singularity at $t=0$. Conditions $\left(\mathrm{C}_{0}\right)$ and $\left(\mathrm{C}_{1}\right)$ restrict the linear part of $L(u)$. We rewrite $L(u)$ in the slightly different form for the later calculations. Denoting $Q(u)+\sum_{h=0}^{m}\left(c_{h}(t, x)-c_{h}(0, x)\right) \vartheta^{h} u$ again by $Q(u)$ and $c_{h}(0, x)$ by $c_{h}(x)$, we can represent $L(u)$ in the form:

$$
\begin{gathered}
L(u)=P(u)+Q(u)+f(t, x), \\
\left\{\begin{array}{l}
P u=\sum_{h=0}^{k} c_{h}(x) \vartheta^{h} u(t, x), Q(u)=\sum_{A \in \mathbb{N}^{*}} q_{A}(u), \\
q_{A}(u)=c_{A}(t, x) \prod_{\alpha \in \Delta(m)}\left(\vartheta^{\alpha_{0}} \partial^{\alpha^{\prime}} u(t, x)\right)^{A_{\alpha},} \\
f(t, x)=L(t, x, 0),
\end{array}\right.
\end{gathered}
$$

where

$$
c_{k}(x) \neq 0 \text { for } x \in \bar{U}^{\prime} \text { and } c_{A}(0, x)=0 \text { for } A \in \mathbb{N}_{l i n}^{M *} \text {. }
$$

Hereafter we assume $L(u)$ is of the form (1.6) with (1.7).

Let $S=U_{0}\left(\theta^{*}\right)$ be a sector in $t$-space and $u(t, x) \in \mathscr{O}\left(S \times U^{\prime}\right)$ be a solution of $L(u)=0$ such that for a constant $\nu_{0}>0$

$$
\sup _{x \in U^{\prime}}|u(t, x)| \leq C|t|^{\nu_{0}}
$$

Let us define an index $\gamma$. Let $e_{A} \in \mathbb{N}$ such that $c_{A}(t, x)=t^{e_{A}} b_{A}(t, x)$ with $b_{A}(0, x) \not \equiv 0$ if $c_{A}(t, x) \not \equiv 0$. An index $\gamma$ is defined by

$$
\gamma=\left\{\begin{array}{l}
\min \left\{\frac{e_{A}+\nu_{0}(|A|-1)}{m_{A}-k} ; A \in \mathbb{N}^{M *}, m_{A}>k\right\} \quad \text { if } k<m, \\
+\infty, \quad \text { if } k=m,
\end{array}\right.
$$

which depends on $\nu_{0}$. If $\nu_{0}$ is improved, then $\gamma$ may be also improved. From the definition

$$
\frac{e_{A}+\nu_{0}(|A|-1)}{\gamma} \geq m_{A}-k .
$$

Remark 1.1. (1) Under the condition (1.8) we have from Proposition 2.5

$$
f(t, x)=\sum_{n \geq \nu_{0}} f_{n}(x) t^{n} .
$$


(2) We give a remark concerning $e_{A}$. We have $e_{A} \geq 1$ for $A \in \mathbb{N}_{l i n}^{M *}$. Once $\gamma$ is defined, we put $e_{A}=0$ for $A$ with $|A| \geq 2$ and $\nu_{0}(|A|-1) / \gamma>m-k$, which gives no influence on the definition of $\gamma$. Hence we may assume $\left\{e_{A} \in \mathbb{N} ; A \in \mathbb{N}^{M *}\right\}$ is bounded and $c_{A}(t, x)=t^{e_{A}} b_{A}(t, x)$ with $b_{A}(t, x) \in \mathscr{O}\left(U_{0} \times U^{\prime}\right)$ such that there is a constant $B$

$$
\left|b_{A}(t, x)\right| \leq B^{|A|} \text { for }(t, x) \in U_{0} \times U^{\prime} .
$$

In order to analyze the singularities of a solution $u(t, x)$ of $L(u)=0$ with (1.8) Mellin transform is available. Let $g(t)$ be a continuous function on $(0, a]$ such that $|g(t)| \leq C|t|^{\nu}$. Then (truncated) Mellin transform $(\mathcal{M} g)(\lambda)$ of $g(t)$ denoted by $\hat{g}(\lambda)$ is defined by

$$
\hat{g}(\lambda):=(\mathcal{M} g)(\lambda)=\int_{0}^{a} t^{\lambda-1} g(t) d t .
$$

The inverse Mellin transform is

$$
\left(\mathcal{M}^{-1} \hat{g}\right)(t)=\frac{1}{2 \pi i} \int_{c-i \infty}^{c+i \infty} t^{-\lambda} \hat{g}(\lambda) d \lambda,
$$

where $c>-\nu$, and $g(t)=\left(\mathcal{M}^{-1} \hat{g}\right)(t)$ holds for $0<t<a$. In this paper we consider functions on sectorial region. Further let $g(t) \in \mathscr{O}\left(U_{0}(\theta)\right), U_{0}=\{|t|<$ $\left.R_{0}\right\}$, with $|g(t)| \leq C|t|^{\nu}$. Let $0<a<R_{0}$ and $|\phi|<\theta$. Then (truncated) Mellin transform $\left(\mathcal{M}^{\phi} g\right)(\lambda)$ of $g(t)$ denoted by $\hat{g}^{\phi}(\lambda)$ is defined by

$$
\hat{g}^{\phi}(\lambda):=\left(\mathcal{M}^{\phi} g\right)(\lambda)=\int_{0}^{a e^{i \phi}} t^{\lambda-1} g(t) d t .
$$

Then

$$
\hat{g}^{\phi}(\lambda)=e^{i \lambda \phi} \int_{0}^{a} s^{\lambda-1} g\left(s e^{i \phi}\right) d s
$$

and

$$
\hat{g}^{\phi, \phi^{\prime}}(\lambda):=\hat{g}^{\phi}(\lambda)-\hat{g}^{\phi^{\prime}}(\lambda)=\int_{a e^{i \phi^{\prime}}}^{a e^{i \phi}} t^{\lambda-1} g(t) d t .
$$

$\hat{g}^{\phi}(\lambda)$ is holomorphic on $\{\lambda \in \mathbb{C} ; \operatorname{Re} \lambda>-\nu\}$ and $\hat{g}^{\phi, \phi^{\prime}}(\lambda)$ is an entire function. The inverse Mellin transform is

$$
\left(\left(\mathcal{M}^{\phi}\right)^{-1} \hat{g}^{\phi}\right)(t)=\frac{1}{2 \pi i} \int_{c-i \infty}^{c+i \infty} t^{-\lambda} \hat{g}^{\phi}(\lambda) d \lambda,
$$

where $c>-\nu$, and $g(t)=\left(\left(\mathcal{M}^{\phi}\right)^{-1} \hat{g}^{\phi}\right)(t)$ holds for $t=|t| e^{i \phi}, 0<|t|<a$. In the following we mainly use $\mathcal{M}^{0}(\phi=0)$, so we denote it simply by $\mathcal{M}$. We give a lemma used in later 
Lemma 1.2. Let $f(t)$ be a continuous function on $[0, a](a>0)$ such that $|f(t)| \leq C|t|^{\kappa_{0}}\left(\kappa_{0}>0\right)$. Let

$$
\psi^{*}(t)=\frac{1}{2 \pi i} \int_{c-i \infty}^{c+i \infty} t^{-\lambda} \frac{(\mathcal{M} f)(\lambda)}{(-\lambda)^{s}} d \lambda \quad\left(-\kappa_{0}<c<0\right)
$$

for $s \in \mathbb{N}$. Then $\psi^{*}(t)$ is a solution of $\vartheta^{s} \psi^{*}(t)=f(t)$ such that $\left|\psi^{*}(t)\right| \leq C|t|^{\kappa_{0}}$.

The proof of Lemma 1.2 is given in Section 3. Let $L(u)=0$ be an equation satisfying (1.7) (equivalently $\left(\mathrm{C}_{0}\right)$ and $\left.\left(\mathrm{C}_{1}\right)\right)$ and $u(t, x)$ be a solution with (1.8). Let us consider its Mellin transform $(\mathcal{M} u)(\lambda, x)$. The first aims are to show that $(\mathcal{M u} u(\lambda, x)$ is extensible to the whole plane as a meromorphic function in $\lambda$ and to determine the location of poles. Set

$$
P(x, \lambda)=\sum_{h=0}^{k} c_{h}(x) \lambda^{h} .
$$

We assume for simplicity for $x \in \bar{U}^{\prime}$

$$
P(x, \lambda) \neq 0 \text { on } \operatorname{Re} \lambda=\nu_{0} .
$$

Hereafter we treat $L(\cdot)$ of the form (1.6) satisfying (1.7) and $\left(\mathrm{C}_{2}\right)$.

Let $\left\{\lambda_{i}(x)\right\}_{i=1}^{k}$ be the roots of $P(x, \lambda)=0\left(x \in U^{\prime}\right)$ such that

$$
\operatorname{Re} \lambda_{i}(x)>\nu_{0} \quad \text { for } 1 \leq i \leq k^{\prime}, \quad \operatorname{Re} \lambda_{i}(x)<\nu_{0} \quad \text { for } i>k^{\prime} .
$$

Set $\lambda_{0}(x)=1$ and define a nonnegative lattice $\Lambda(x)$ generated by $\left\{\lambda_{i}(x)\right\}_{i=0}^{k^{\prime}}$,

$$
\Lambda(x):=\left\{\lambda=-\sum_{i=0}^{k^{\prime}} n_{i} \lambda_{i}(x) ;\left(n_{0}, \cdots, n_{k^{\prime}}\right) \in \mathbb{N}^{k^{\prime}+1}\right\}
$$

and its subset $\Lambda_{-\nu_{0}}(x)$

$$
\Lambda_{-\nu_{0}}(x)=\Lambda(x) \cap\left\{\operatorname{Re} \lambda \leq-\nu_{0}\right\} .
$$

Let $u(t, x)$ be a solution of $L(u)=0$ with bound (1.8) and consider the Mellin transform of $u(t, x)$

$$
(\mathcal{M} u)(\lambda, x)=\int_{0}^{a} t^{\lambda-1} u(t, x) d t,
$$

which is holomorphic $\left\{\lambda ; \operatorname{Re} \lambda>-\nu_{0}\right\}$. We give one of main results which is a clue to study the singularities of $u(t, x)$. 
Theorem 1.3. $\quad(\mathcal{M} u)(\lambda, x)$ is meromorphic in $\lambda$ on the whole plane and its poles are contained in $\Lambda_{-\nu_{0}}(x)$.

The same result as Theorem 1.3 holds for $\left(\mathcal{M}^{\phi} u\right)(\lambda, x)(|\phi|<\theta)$. By the inverse Mellin transform

$$
u(t, x)=\frac{1}{2 \pi i} \int_{c-i \infty}^{c+i \infty} t^{-\lambda}(\mathcal{M} u)(\lambda, x) d \lambda \quad\left(-\nu_{0}<c\right) .
$$

The singularities of $(\mathcal{M} u)(\lambda, x)$ essentially contribute to the integral (1.25). Theorem 1.3 gives us informations about them. So by calculating the integral (1.25), we can obtain the asymptotic terms of a singular solution $u(t, x)$ near $\{t=0\}$. In order to describe asymptotic behaviors of singular solutions in details let us introduce a class $\mathscr{M}_{\varphi}\left(U^{\prime}\right)$ of holomorphic functions. $\mathscr{M}_{\varphi}\left(U^{\prime}\right)$ is a subspace of $\mathscr{O}\left((\widetilde{\mathbb{C} \backslash\{0\}}) \times U^{\prime}\right)$ whose elements are the image of the inverse Mellin transform of rational functions.

Definition 1.4. (1). Let $\varphi(\lambda, x) \in \mathscr{O}\left(U^{\prime}\right)[\lambda]$ with nonvanishing leading term. $\mathscr{M}_{\varphi}\left(U^{\prime}\right)$ is the set of all $w(t, x) \in \mathscr{O}\left((\widetilde{\mathbb{C} \backslash\{0\}}) \times U^{\prime}\right)$ represented in the form

$$
w(t, x)=\frac{1}{2 \pi i} \int_{\mathcal{C}} t^{-\lambda} \frac{\psi(\lambda, x)}{\varphi(\lambda, x)} d \lambda,
$$

where $\psi(\lambda, x) \in \mathscr{O}\left(U^{\prime}\right)[\lambda]$ with $\operatorname{deg} \psi<\operatorname{deg} \varphi$ and $\mathcal{C}$ is a Jordan curve enclosing all the zeros of $\varphi(\lambda, x)$.

If all the zeros $\left\{a_{j}(x)\right\}_{j=1}^{s}$ of $\varphi(\lambda, x)$ are distinct, then there exist holomorphic functions $\left\{\omega_{j}(x)\right\}_{j=1}^{s}$ such that

$$
w(t, x)=\sum_{j=1}^{s} t^{-a_{j}(x)} \omega_{j}(x) .
$$

Set $\mathscr{M}_{\text {rat }}\left(U^{\prime}\right)=\cup_{\varphi} \mathscr{M}_{\varphi}\left(U^{\prime}\right)$. We show that the asymptotic terms of a singular solution $u(t, x)$ are given by functions in $\mathscr{M}_{\text {rat }}\left(U^{\prime}\right)$ and estimate the remainder. The following is the other main result.

Theorem 1.5. Suppose that $L(u)$ satisfies $\left(C_{0}\right),\left(C_{1}\right),\left(C_{2}\right)$. Let $u(t, x) \in$ $\mathscr{O}\left(S \times U^{\prime}\right)$ with bound (1.8) be a solution of $L(u)=0$. Then there exist $u_{n}(t, x) \in$ $\mathscr{M}_{\varphi_{n}}\left(W^{\prime}\right) \quad(n \in \mathbb{N})$ in a neighborhood $W^{\prime}$ of $x=0$ such that for any sector 
$T \Subset S$

$$
\left\{\begin{array}{c}
\left|u_{n}(t, x)\right| \leq C_{0} C^{n} \Gamma\left(\frac{p_{n}}{\gamma}+1\right)|t|^{p_{n}+\nu_{0}}, \\
\left|u(t, x)-\sum_{n=0}^{N-1} u_{n}(t, x)\right| \leq C_{0} C^{N} \Gamma\left(\frac{p_{N}}{\gamma}+1\right)|t|^{p_{N}+\nu_{0}}
\end{array}\right.
$$

holds for $(t, x) \in T \times W^{\prime}$ and $N \in \mathbb{N}$, where if $\gamma<+\infty, p_{n}=\frac{\gamma n}{p}$ for some positive integer $p$ and if $\gamma=+\infty, p_{n}=c n$ for some $c>0$. As for the zeros of $\varphi_{n}(\lambda, x) \in \mathscr{O}\left(W^{\prime}\right)[\lambda]$ there exist $a, b>0$ such that

$$
\left\{\lambda ; \varphi_{n}(\lambda, x)=0\right\} \subset \Lambda_{-\nu_{0}}(x) \cap\{R e ; \lambda>-(a n+b)\} .
$$

Theorem 1.5 implies that $u(t, x)$ has an asymptotic expansion with asymptotic terms $\left\{u_{n}(t, x)\right\}_{n \in \mathbb{N}} \subset \mathscr{M}_{\text {rat }}(W)$,

$$
u(t, x) \sim \sum_{n=0}^{\infty} u_{n}(t, x)
$$

in the sense of (1.27). The remainder estimate in (1.27) is called Gevrey type. We devote the rest of the paper to the proof of Theorems 1.3 and 1.5.

Remark 1.6. (1) The case $m=k$ was studied in [1] and [11]. If $m=k$, then $\gamma=+\infty$ and $\{t=0\}$ is, so called, of regular singular type. Theorem 1.5 implies that $u(t, x)=\sum_{n=0}^{+\infty} u_{n}(t . x)$ converges, which was shown in the above papers. If $m>k$, then $\gamma$ is finite and Theorem 1.5 is a generalization to equations with an irregular singular surface $\{t=0\}$.

(2) Mellin type integral was available to analyze singularity of solutions when the algebraic equation $P(x, \lambda)=0$ with respect to $\lambda$ (see (1.20)) has multiple roots. It was used for linear equations in [3] and [8]. In [3] the case $m=k$, so called Fuchsian equations, and in [8] more general case $k \leq m$ were studied and the structure and asymptotic behaviors of singular solutions were obtained.

Let us give an example.

$$
t^{k} \partial_{t}^{k} u=A\left(t, x,\left(t \partial_{t}\right)^{\alpha_{0}} \partial_{x}^{\alpha^{\prime}} u ;|\alpha| \leq m, \alpha_{0} \leq k-1\right),
$$

where $A(t, x, Z), Z=\left(Z_{\alpha} ;|\alpha| \leq m\right)$, is holomorphic in a neighborhood $U_{0} \times$ $U^{\prime} \times \Omega$ of $(t, x, Z)=(0,0,0)$. Let

$$
A_{l i n}(t, x, Z)=\sum_{\alpha} \frac{\partial A}{\partial Z_{\alpha}}(t, x, 0) Z_{\alpha}
$$


be the linearization of $A(t, x, Z)$ at $Z=0$. Assume

$$
\frac{\partial A}{\partial Z_{\alpha}}(0, x, 0)=0 \quad \text { for } \alpha \text { with } \alpha^{\prime} \neq 0 .
$$

Then

$$
P(x, \lambda)=\lambda(\lambda-1) \cdots(\lambda-k+1)-\sum_{\alpha_{0}=0}^{k-1} \frac{\partial A}{\partial Z_{\left(\alpha_{0}, 0\right)}}(0, x, 0) \lambda^{\alpha_{0}}
$$

and conditions $\left(\mathrm{C}_{0}\right)$ and $\left(\mathrm{C}_{1}\right)$ are satisfied, and we can apply Theorem 1.5 to solutions of (1.29).

We give another example which is reduced to (1.29). Consider

$$
\partial_{t}^{k} u=A\left(t, x, \partial_{t}^{\alpha_{0}} \partial_{x}^{\alpha^{\prime}} u ;|\alpha| \leq m, \alpha_{0} \leq k-1\right) \quad(1 \leq k \leq m),
$$

where $A\left(t, x, \partial_{t}^{\alpha_{0}} \partial_{x}^{\alpha^{\prime}} u\right)$ is a partial differential operator with order $m$ and $A(t, x$, $p), p=\left(p_{\alpha} ;|\alpha| \leq m\right)$, is holomorphic in a neighborhood $U_{0} \times U^{\prime} \times \Pi$ of $(t, x, p)=(0,0,0)$. Let $u(t, x)$ be a solution of (1.33) in a sectorial region $S \times U^{\prime}$ such that $|u(t, x)| \leq C|t|^{k-1+\varepsilon}$ for some $\varepsilon>0$. Let $v(t, x)=t^{-k+1} u(t, x)$. Then $v(t, x)$ satisfies $|v(t, x)| \leq C|t|^{\varepsilon}$,

$$
\begin{gathered}
t \partial_{t}^{k}\left(t^{k-1} v\right)=t A\left(t, x, \partial_{t}^{\alpha_{0}} \partial_{x}^{\alpha^{\prime}} t^{k-1} v\right), \\
P(x, \lambda)=(\lambda+k-1)(\lambda+k-2) \cdots(\lambda+1) \lambda
\end{gathered}
$$

and conditions $\left(\mathrm{C}_{0}\right),\left(\mathrm{C}_{1}\right)$ and $\left(\mathrm{C}_{2}\right)$ hold.

\section{§2. Majorant Functions 1}

In this paper we estimate many functions. For this purpose, majorant functions are indispensable. Let $A(w)=\sum_{\alpha} A_{\alpha} w^{\alpha}$ and $B(w)=\sum_{\alpha} B_{\alpha} w^{\alpha}$ be formal power series of $n$ variables $w=\left(w_{1}, \ldots, w_{n}\right)$. $A(w) \ll B(w)$ means $\left|A_{\alpha}\right| \leq B_{\alpha}$ for all $\alpha \in \mathbb{N}^{n}$ and $A(w) \gg 0$ means $A_{\alpha} \geq 0$ for all $\alpha$. Let $w^{*}=$ $\left(w_{1}^{*}, \ldots, w_{n}^{*}\right) \in \mathbb{C}^{n}$. If we consider formal power series at $w=w^{*}$, we use the notation $A\left(w-w^{*}\right) \ll B\left(w-w^{*}\right)$, which means $\left|A_{\alpha}\right| \leq B_{\alpha}$ for all $\alpha$. The proofs of Lemmas and Propositions in this section are given in Section 7.

Let $m \in \mathbb{N}$ and set

$$
\theta(X)=c \sum_{n=0}^{+\infty} \frac{X^{n}}{(n+1)^{m+2}},
$$

where $c>0$ and it is fixed later. $\theta(X)$ or its modifications are used in [2], [4], [9] and [12]. We have $\theta^{(s)}(X)=c \sum_{n=0}^{+\infty} \frac{(n+s)(n+s-1) \cdots(n+1)}{(n+s+1)^{m+2}} X^{n}=$ $c \sum_{n=0}^{+\infty} \frac{(n+s) !}{(n+s+1)^{m+2} n !} X^{n}$. The following properties are important. 
Lemma 2.1. (1) Let $0<r^{\prime}<1$. Then there is a constant $C=C\left(r^{\prime}\right)$ such that

$$
\theta(X) \ll \frac{c}{1-X} \ll C \theta\left(X / r^{\prime}\right)
$$

$$
(p+1) \theta^{(p)}(X) \ll 2^{m+2} \theta^{(p+1)}(X) .
$$

(3) Let $\ell_{1}, \ell_{2} \in \mathbb{N}$ such that $0 \leq \ell_{1} \leq \ell_{2} \leq m$. Then there is a constant $c>0$ in (2.1) such that

$$
\theta^{\left(\ell_{1}\right)}(X) \theta^{\left(\ell_{2}\right)}(X) \ll \theta^{\left(\ell_{2}\right)}(X)
$$

In the following we fix $c>0$ in (2.1) so that (2.4) holds.

Lemma 2.2. Let $\ell_{i} \in \mathbb{N}(1 \leq i \leq r)$ with $0 \leq \ell_{i} \leq m$ and $p_{i} \in \mathbb{N}(1 \leq$ $i \leq r)$. Set $\ell^{\prime}=\max _{1 \leq i \leq r} \ell_{i}$ and $p=\sum_{i=1}^{r} p_{i}$. Then

$$
\sum_{\substack{\left(q_{1}, q_{2}, \cdots, q_{r}\right) \in \mathbb{N}^{r} \\ q_{1}+q_{2}+\cdots+q_{r}=q}} \prod_{i=1}^{r} \frac{\theta^{\left(p_{i}+q_{i}+\ell_{i}\right)}(X)}{q_{i} !} \ll \frac{\theta^{\left(p+q+\ell^{\prime}\right)}(X)}{q !} .
$$

Define for $0<R<1$

$$
\Theta_{-q}^{(p)}(R ; X)=\frac{1}{q !} \frac{d^{p}}{d X^{p}} \theta\left(\frac{X}{R}\right)=\frac{1}{R^{p} q !} \theta^{(p)}\left(\frac{X}{R}\right) .
$$

From Lemmas 2.1 and 2.2 we have

Proposition 2.3. (1) Let $0<r<R<1$. Then there exists a constant $C=C(r / R)$ such that

$$
\left\{\begin{array}{l}
\Theta_{0}^{(p)}(R ; X) \ll \frac{c p !}{R^{p}\left(1-\frac{X}{R}\right)^{p+1}} \ll C \Theta_{0}^{(p)}(r ; X), \\
p ! \Theta_{0}(R ; X) \ll C \Theta_{0}^{(p)}(r ; X) .
\end{array}\right.
$$

(2) Let $\ell_{1}, \ell_{2} \in \mathbb{N}$ with $\ell_{1} \leq \ell_{2} \leq m$. Then

$$
\Theta_{0}^{\left(\ell_{1}\right)}(R ; X) \Theta_{0}^{\left(\ell_{2}\right)}(R ; X) \ll R^{-\ell_{1}} \Theta_{0}^{\left(\ell_{2}\right)}(R ; X) .
$$


(3) There is a constant $C>0$ such that

$$
(p+1) \Theta_{-q}^{(p)}(R ; X) \ll C R \Theta_{-q}^{(p+1)}(R ; X) .
$$

In particular, for $0<c<1$ there is $0<R<1$ such that

$$
(p+1) \Theta_{-q}^{(p)}(R ; X) \ll c \Theta_{-q}^{(p+1)}(R ; X) .
$$

(4) For $0<R<R^{\prime}$

$$
\Theta_{-q}^{(p)}\left(R^{\prime} ; X\right) \ll\left(\frac{R}{R^{\prime}}\right)^{p} \Theta_{-q}^{(p)}(R ; X) .
$$

(5) If $|X| \leq \frac{R}{2}$, there is a constant $C$ such that

$$
\left|\Theta_{-q}^{(p)}(R ; X)\right| \leq \frac{C 2^{p} p !}{R^{p} q !} .
$$

Proposition 2.4. (1) Let $l$ be an integer with $0 \leq l \leq m$. Then

$$
\sum_{\substack{\left(n_{1}, n_{2} \cdots, n_{r}\right) \in \mathbb{N}^{r} \\ n_{1}+n_{2}+\cdots+n_{r}=n^{2}}} \frac{\Theta_{0}^{\left(n_{1}+l\right)}(R ; X) \Theta_{0}^{\left(n_{2}+l\right)}(R ; X) \cdots \Theta_{0}^{\left(n_{r}+l\right)}(R ; X)}{n_{1} ! n_{2} ! \cdots n_{r} !} \ll \frac{\Theta_{0}^{(n+l)}(R ; X)}{R^{l(r-1)} n !} .
$$

(2) Let $\ell_{i} \in \mathbb{N}$ with $0 \leq \ell_{i} \leq m$ and $p_{i} \in \mathbb{N}(1 \leq i \leq r)$. Set $\ell^{\prime}=\max _{1 \leq i \leq r} \ell_{i}$ and $p=\sum_{i=1}^{r} p_{i}$. Then

$$
\sum_{\left\{\begin{array}{c}
\left(q_{1}, q_{2}, \cdots, q_{r}\right) \in \mathbb{N}^{r} \\
q_{1}+q_{2}, \cdots+q_{r}=q
\end{array}\right.} \prod_{i=1}^{r} \Theta_{-q_{i}}^{\left(p_{i}+q_{i}+\ell_{i}\right)}(R ; X) \ll \frac{1}{R^{\ell^{\prime}(r-1)}} \Theta_{-q}^{\left(p+q+\ell^{\prime}\right)}(R ; X) .
$$

(3) Let $k \in \mathbb{N}, 0 \leq \ell \leq m$ and $s \geq 0$. Further suppose that $k \geq 1$ if $s=0$, and $R>0$ is small. Then there is a constant $C>0$ such that

$$
\begin{gathered}
\sum_{\left\{\begin{array}{c}
\left(n_{1}, n_{2} \cdots, n_{r}\right) \in \mathbb{N}^{r} \\
n_{1}+n_{2}+\cdots+n_{r}=n
\end{array}\right\}} \prod_{i=1}^{r} \Theta_{-\left[s n_{i}\right]-k n_{i}}^{\left(\left[s n_{i}\right]+k n_{i}+\ell\right)}(R ; X) \ll \frac{C}{R^{\ell(r-1)}} \Theta_{-[s n]-k n}^{([s n]+k n+\ell)}(R ; X), \\
\sum_{\left\{\begin{array}{c}
\left(n_{1}, n_{2} \cdots, n_{r}\right) \in \mathbb{N}^{r} \\
n_{1}+n_{2}+\cdots+n_{r}=n
\end{array}\right\}} \prod_{i=1}^{r} \Theta_{-k n_{i}}^{\left(\left[s n_{i}\right]+k n_{i}+\ell\right)}(R ; X) \ll \frac{C}{R^{\ell(r-1)}} \Theta_{-k n}^{([s n]+k n+\ell)}(R ; X) .
\end{gathered}
$$


(4) Let $0<r<R$ and $0<\delta<R-r$. Then there is a constant $C=C(\delta, r)$ such that

$$
\sum_{i=s}^{\infty} \delta^{i-s} \Theta_{-i}^{(i)}(R ; X) \ll C \Theta_{-s}^{(s)}(r ; X)
$$

Next let us proceed to introduce another majorant method to estimate functions on sectorial regions. Let $S=\left\{t \in \mathbb{C} ; 0<|t|<T^{*},|\arg t|<\theta^{*}\right\}$. Let $0<T_{0}<T_{1}<T^{*}$ and $0<\theta_{0}<\theta_{1}<\theta^{*}$ with $\theta_{1}-\theta_{0}<\pi / 6$. For $0 \leq \tau \leq 1$ define

$$
S^{\tau}=\left\{t \in \mathbb{C} ; 0<|t|<(1-\tau) T_{0}+\tau T_{1},|\arg t|<(1-\tau) \theta_{0}+\tau \theta_{1}\right\} .
$$

Then $S^{0} \subset S^{\tau} \subset S^{1} \subset S$.

Proposition 2.5. Let $f(t) \in \mathscr{O}(S)$.

(1) Suppose that for any $0<\tau<1$

$$
|f(t)| \leq \frac{M|t|^{s}}{(1-\tau)^{q}} \quad(q \geq 0) \quad \text { for } t \in S^{\tau} .
$$

Then there exist constants $c$ and $C$ which are independent of $\tau$ such that

$$
|\vartheta f(t)| \leq \frac{C(q+1) \exp \frac{c s}{q+1}}{(1-\tau)^{q+1}} M|t|^{s} \quad \text { for } t \in S^{\tau} .
$$

(2) Let $0<t_{0}<t_{1}<T_{0}$. Suppose that for any $0<\tau<1$

$$
|f(t)| \leq \frac{M|t|^{s}}{(1-\tau)^{q}} \quad(q \geq 0) \quad \text { for } t \in S^{\tau} \cap\left\{|t| \leq t_{1}\right\} .
$$

Then there exist constants $c$ and $C$ which are independent of $t_{0}, t_{1}$ and $\tau$ such that

$$
\left|t^{n} f^{(n)}(t)\right| \leq \frac{M C^{n} n ! e^{\frac{c s}{q+1}}|t|^{s}}{(1-\tau)^{q}} \max \left\{\left(\frac{q+1}{1-\tau}\right)^{n},\left(\frac{t_{0}}{t_{1}-t_{0}}\right)^{n}\right\}
$$

for $t \in S^{\tau} \cap\left\{|t| \leq t_{0}\right\}$.

Corollary 2.6. Let $f(t) \in \mathscr{O}(S)$. Suppose that estimate (2.17) holds for any $0<\tau<1$. Then there exist constants $c$ and $C$ which are independent of $\tau$ such that

$$
\left|\vartheta^{n} f(t)\right| \leq \frac{C^{n} \prod_{i=1}^{n}(q+i) \exp \frac{c s}{q+i}}{(1-\tau)^{q+n}} M|t|^{s} \quad \text { for } t \in S^{\tau} .
$$


By using the above majorant functions we have an estimate of products of holomorphic functions on a sectorial region.

Proposition 2.7. Let $x^{*}=\left(x_{1}^{*}, \cdots, x_{d}^{*}\right) \in U^{\prime}$ and $X-X^{*}=\sum_{i=1}^{d}\left(x_{i}-\right.$ $\left.x_{i}^{*}\right)$. Suppose $u_{i}(t, x) \in \mathscr{O}\left(S \times U^{\prime}\right)(1 \leq i \leq \ell)$ with $u_{i}(t, x) \underset{x^{*}}{\ll} C_{0}|t|^{s_{i}} \Theta(r ; X-$ $\left.X^{*}\right)\left(s_{i} \geq 0\right)$ and $b(t, x) \in \mathscr{O}\left(S \times U^{\prime}\right)$ with $b(t, x) \underset{x^{*}}{\ll} B \Theta\left(r^{\prime} ; X-X^{*}\right)\left(r^{\prime} \geq r\right)$. Let $\alpha(i) \in \Delta(m)(1 \leq i \leq \ell)$. Then for any subsector $T \Subset S^{1}$ there exists a constant $C=C(T)>0$ such that for $t \in T$

$$
b(t, x) \prod_{i=1}^{\ell} \vartheta^{\alpha_{0}(i)} \partial^{\alpha^{\prime}(i)} u_{i}(t, x) \underset{x^{*}}{\ll} B C^{\ell+s}|t|^{s} \Theta^{(m)}\left(r ; X-X^{*}\right),
$$

where $s=\sum_{i=1}^{\ell} s_{i}$.

\section{§3. Mellin Transform of Singular Solutions}

Let $L(\cdot)$ be an operator in the form (1.6) and satisfying (1.7) and $\left(\mathrm{C}_{2}\right)$. Consider a solution $u(t, x) \in \mathscr{O}\left(S \times U^{\prime}\right)$ with bound (1.8). The purpose of this section is to show Theorem 1.3, that is, to prolong $(\mathcal{M u} u(\lambda, x)$ as a meromorphic function in $\lambda$ to the whole plane.

First we give lemmas concerning $\mathscr{M}_{\varphi}\left(U^{\prime}\right)$ for this purpose. Let $\varphi_{i}(t, x) \in$ $\mathcal{O}\left(U^{\prime}\right)[\lambda](i=1,2)$ whose leading term is $a_{i}(x) \neq 0$ in $U^{\prime}$. By factorizing, $\varphi_{i}(t, x)=a_{i}(x) \prod_{j=1}^{p_{i}}\left(\lambda-a_{i}^{j}(x)\right)$, where each of $\left\{a_{i}^{j}(x)\right\}_{j=1}^{p_{i}}$ is not necessarily holomorphic. Define

$$
\left(\varphi_{1} \# \varphi_{2}\right)(\lambda, x)=a_{1}(x)^{p_{2}} a_{2}(x)^{p_{1}} \prod_{j_{1}=1}^{p_{1}} \prod_{j_{2}=1}^{p_{2}}\left(\lambda-a_{1}^{j_{1}}(x)-a_{2}^{j_{2}}(x)\right) .
$$

Then $\left(\varphi_{1} \# \varphi_{2}\right)=a_{2}(x)^{p_{1}} \prod_{j=1}^{p_{2}} \varphi_{1}\left(\lambda-a_{2}^{j}(x), x\right)=a_{1}(x)^{p_{2}} \prod_{j=1}^{p_{1}} \varphi_{2}\left(\lambda-a_{1}^{j}(x), x\right)$

Lemma 3.1. Let $w_{i}(t, x) \in \mathscr{M}_{\varphi_{i}}\left(U^{\prime}\right)(i=1,2)$. Then $w(t, x)=w_{1}(t$, $x) w_{2}(t, x) \in \mathscr{M}_{\varphi_{1} \# \varphi_{2}}(U)$.

Lemma 3.2. Let $w(t, x) \in \mathscr{M}_{\varphi}\left(U^{\prime}\right)$ with $w(t, x)=\frac{1}{2 \pi i} \int_{\mathcal{C}} t^{-\lambda} \frac{\psi(\lambda, x)}{\varphi(\lambda, x)} d \lambda$. Then

$$
(\mathcal{M} w)(\lambda, x)=\frac{\psi(\lambda, x)}{\varphi(\lambda, x)}+\text { an entire function }
$$


The proofs of Lemmas 3.1 and 3.2 are given in the final part of this section. Let us return to (1.6) and (1.7). Recall $P(x, \lambda)=\sum_{h} c_{h}(x) \lambda^{h}$ with the roots $\left\{\lambda_{i}(x)\right\}_{i=1}^{k}$ such that

$$
\operatorname{Re} \lambda_{i}(x)>\nu_{0} \quad \text { for } 1 \leq i \leq k^{\prime}, \quad \operatorname{Re} \lambda_{i}(x)<\nu_{0} \quad \text { for } i>k^{\prime} .
$$

Set

$$
\Phi(\lambda, x)=\prod_{i=1}^{k^{\prime}}\left(\lambda+\lambda_{i}(x)\right)
$$

Then

$$
P(x,-\lambda)=(-1)^{k} \Phi(\lambda, x) \prod_{i>k^{\prime}}\left(\lambda+\lambda_{i}(x)\right) .
$$

Set

$$
\nu_{*}=\min _{A}\left\{e_{A}+\nu_{0}(|A|-1)\right\}
$$

and for $n \in \mathbb{N}$

$$
\hat{\nu}_{n}=\nu_{0}+n \nu_{*} .
$$

From (1.7) $\nu_{*} \geq \min \left\{1, \nu_{0}\right\}>0$ holds. $\Lambda(x)$ is a nonnegative lattice generated by $\left\{\lambda_{i}(x)\right\}_{i=0}^{k^{\prime}}$, where $\lambda_{0}(x)=1$,

$$
\Lambda(x)=\left\{\lambda=-\sum_{i=0}^{k^{\prime}} n_{i} \lambda_{i}(x) ;\left(n_{0}, \cdots, n_{k^{\prime}}\right) \in \mathbb{N}^{k^{\prime}+1}\right\}
$$

and $\Lambda_{-\nu_{0}}(x)=\Lambda(x) \cap\left\{\operatorname{Re} \lambda \leq-\nu_{0}\right\}$.

Lemma 3.3. Let $g(t, x) \in \mathscr{O}\left(S \times U^{\prime}\right)$ with $|g(t, x)| \leq K|t|^{\kappa_{0}}$. Then $(\mathcal{M g})(\lambda, x)$ is holomorphic in $\left\{\lambda ; \operatorname{Re} \lambda>-\kappa_{0}\right\}$ and

(1)

$$
\left(\mathcal{M} \vartheta^{h} g\right)(\lambda, x)=(-\lambda)^{h}(\mathcal{M} g)(\lambda, x)+a^{\lambda} H_{1}(\lambda, x),
$$

where $H_{1}(\lambda, x)$ is a polynomial in $\lambda$ with degree $\leq h-1$.

(2) Further assume $g(t, x) \in \mathscr{M}_{\varphi}\left(U^{\prime}\right)$, where $\left\{\lambda ; \varphi(\lambda, x)=0, x \in U^{\prime}\right\} \subset$ $\Lambda_{-\nu_{0}}(x)$. Let $Q(\cdot)$ be that in (1.6). Then $(\mathcal{M} g)(\lambda, x)$ and $(\mathcal{M} Q(g))(\lambda, x)$ are meromorphic on the whole plane and their poles are in $\Lambda_{-\nu_{0}}(x)$. 
Proof. (1) By integrations by parts

$$
\begin{aligned}
& \int_{0}^{a} t^{\lambda-1} \vartheta^{h} g(t, x) d \lambda=a^{\lambda}\left(\vartheta^{h-1} g\right)(a, x)-\lambda \int_{0}^{a} t^{\lambda-1} \vartheta^{h-1} g(t, x) d \lambda \\
& =a^{\lambda}\left(\sum_{\ell=1}^{h}(-\lambda)^{\ell-1}\left(\vartheta^{h-\ell} g\right)(a, x)\right)+(-\lambda)^{h} \int_{0}^{a} t^{\lambda-1} g(t, x) d \lambda,
\end{aligned}
$$

hence we have (3.8).

(2). The assertion about $(\mathcal{M g})(\lambda, x)$ follows from Lemma 3.2. By Lemma 3.1 $\prod_{\alpha \in \Delta(m)}\left(\vartheta^{\alpha_{0}} \partial^{\alpha^{\prime}} g(t, x)\right)^{A_{\alpha}} \in \mathcal{M}_{\varphi_{A}}\left(U^{\prime}\right)$, where $\varphi_{A}=\overbrace{\varphi \# \cdots \# \varphi}^{p}, p \leq$ $\sum_{\alpha \in \Delta(m)}\left(\left|\alpha^{\prime}\right|+1\right) A_{\alpha}$, and $\left\{\varphi_{A}(\lambda, x)=0\right\} \subset \Lambda_{-\nu_{0}}(x) . c_{A}(t, x)$ is holomorphic at $t=0$, hence $\left(\mathcal{M} c_{A}\right)(\lambda, x)$ has at most single poles at $\lambda=0,-1, \cdots$. So the Mellin transform of $c_{A}(t, x) \prod_{\alpha \in \Delta(m)}\left(\vartheta^{\alpha_{0}} \partial^{\alpha^{\prime}} g(t, x)\right)^{A_{\alpha}}$ is meromorphic on the whole plane except on $\Lambda_{-\nu_{0}}(x)$ and that of $Q(g)$ is so.

Now let $u(t, x) \in \mathscr{O}\left(S \times U^{\prime}\right)$ solve $L(u)=0$ with bound (1.8). Let us extend $(\mathcal{M} u)(\lambda, x)$ to the left half plane $\left\{\operatorname{Re} \lambda \leq-\nu_{0}\right\}$. For this purpose some lemmas are given.

Lemma 3.4. (1) $(\mathcal{M} f)(\lambda, x)$ is meromorphic in $\lambda$ on the whole plane and its poles are in $\left\{\lambda \in-\mathbb{N} ; \operatorname{Re} \lambda \leq-\nu_{0}\right\}$.

(2) $(\mathcal{M Q} Q(u))(\lambda, x)$ is holomorphic in $\left\{\lambda \in \mathbb{C} ; \operatorname{Re} \lambda>-\hat{\nu}_{1}\right\}$ and

$$
|(\mathcal{M} Q(u))(\lambda, x)| \leq C_{1} a^{\operatorname{Re\lambda }} /\left(\operatorname{Re} \lambda+\hat{\nu}_{1}\right),
$$

where $C_{1}$ is locally uniform in $x$.

Proof. (1) The Mellin transforms of holomorphic functions at $t=0$ are meromorphic in $\lambda$ on the whole plane and its poles are in $\{\lambda \in-\mathbb{N}\}$. By Remark 1.1-(1) $(\mathcal{M} f)(\lambda, x)$ is holomorphic $\left\{\lambda ; \operatorname{Re} \lambda>-\nu_{0}\right\}$. Hence the assertion (1) holds.

(2) Let $x^{*}=\left(x_{1}^{*}, \cdots, x_{d}^{*}\right) \in U^{\prime}$. Then there exist $C, r>0$ depending on $x^{*}$ such that $u(t, x) \underset{x^{*}}{\ll} C|t|^{\nu_{0}} \Theta\left(r ; X-X^{*}\right), X-X^{*}=\sum_{i=1}^{d}\left(x_{i}-x_{i}^{*}\right)$. It follows from (1.12) and Proposition 2.7 that for any $T \Subset S$ there is a constant $C_{0}$ such that for $t \in T$

$$
t^{e_{A}} b_{A}(t, x) \prod_{\alpha \in \Delta(m)}\left(\vartheta_{t}^{\alpha_{0}} \partial_{x}^{\alpha^{\prime}} u(t, x)\right)^{A_{\alpha}} \underset{x^{*}}{\ll} C_{0}^{|A|}|t|^{e_{A}+\nu_{0}|A|} \Theta^{(m)}\left(r ; X-X^{*}\right) .
$$

From (3.5) there is a small $t_{0}>0$ such that if $t \in T$ with $|t|<t_{0}$,

$$
\sum_{A} C_{0}^{|A|}|t|^{e_{A}+\nu_{0}|A|}=|t|^{\nu_{0}} \sum_{A} C_{0}^{|A|}|t|^{e_{A}+\nu_{0}(|A|-1)} \leq C_{1}|t|^{\nu^{*}+\nu_{0}}
$$


and $Q(u) \underset{x^{*}}{\ll} C_{1}|t|^{\nu^{*}+\nu_{0}} \Theta^{(m)}\left(r ; X-X^{*}\right)$. Therefore $(\mathcal{M} Q(u))(\lambda, x)$ is holomorphic in $\lambda$ in $\left\{\operatorname{Re} \lambda>-\hat{\nu}_{1}\right\}\left(\hat{\nu}_{1}=\nu_{*}+\nu_{0}\right)$ and the estimate holds.

Lemma 3.5. (1) $(\mathcal{M} u)(\lambda, x)$ is meromorphic in $\left\{\lambda \in \mathbb{C} ; \operatorname{Re} \lambda>-\hat{\nu}_{1}\right\}$, whose poles are contained in $\Lambda_{-\nu_{0}}(x)$.

(2) Let $\tilde{\nu}<\hat{\nu}_{1}$ and $L>0$ be a large constant such that $\{\lambda ; \operatorname{Re} \lambda>-\tilde{\nu},|\lambda| \geq$ $L\} \cap \Lambda_{-\nu_{0}}(x)=\emptyset$ for $x \in U^{\prime}$. Then there exists a constant $C$ which is locally uniform in $x$ and depends on $\tilde{\nu}$ such that for $\lambda \in\{\lambda ; \operatorname{Re} \lambda>-\tilde{\nu},|\lambda| \geq L\}$

$$
|(\mathcal{M} u)(\lambda, x)| \leq C a^{R e \lambda} / \operatorname{Re} \lambda .
$$

Proof. From Lemma 3.3

$$
\begin{aligned}
& 0=(\mathcal{M P u})(\lambda, x)+(\mathcal{M} Q(u))(\lambda, x)+(\mathcal{M} f)(\lambda, x) \\
& =P(x,-\lambda)(\mathcal{M} u)(\lambda, x)+a^{\lambda} H(\lambda, x)+(\mathcal{M} Q(u))(\lambda, x)+(\mathcal{M} f)(\lambda, x) .
\end{aligned}
$$

Hence

$$
(\mathcal{M} u)(\lambda, x)=-\frac{a^{\lambda} H(\lambda, x)+(\mathcal{M} Q(u))(\lambda, x)+(\mathcal{M} f)(\lambda, x)}{P(x,-\lambda)},
$$

whose numerator is meromorphic in $\left\{\lambda \in \mathbb{C} ; \operatorname{Re} \lambda>-\hat{\nu}_{1}\right\}$, and its poles are those of $(\mathcal{M} f)(\lambda, x)$ by Lemma 3.4. Therefore $(\mathcal{M} u)(\lambda, x)$ is meromorphic in $\left\{\operatorname{Re} \lambda>-\hat{\nu}_{1}\right\}$. However it is holomorphic in $\left\{\operatorname{Re} \lambda>-\nu_{0}\right\}$, so its poles in $\left\{\operatorname{Re} \lambda>-\hat{\nu}_{1}\right\}$ are in $(\{\lambda ; P(x,-\lambda)=0\} \cup\{-\mathbb{N}\}) \cap\left\{\operatorname{Re} \lambda \leq-\nu_{0}\right\} \subset \Lambda_{-\nu_{0}}(x)$. We have (3.9) from (3.10) and Lemma 3.4.

Let us show that $(\mathcal{M} u)(\lambda, x)$ is meromorphically extensible to $\{\lambda \in \mathbb{C}$; $\left.\operatorname{Re} \lambda>-\hat{\nu}_{2}\right\}$. Let $x^{*} \in U^{\prime}$ and $\nu^{\prime}$ be arbitray with $\nu_{0}<\nu^{\prime}<\hat{\nu}_{1}$. Then there exist a neighborhood $U^{\prime}\left(x^{*}\right)$ of $x^{*}$, a $\nu$ with $\nu^{\prime}<\nu<\hat{\nu}_{1}$ and a small $\epsilon>0$ such that $(\mathcal{M} u)(\lambda, x)$ is holomorphic in $(\lambda, x) \in\{|\lambda+\nu|<\epsilon\} \times U^{\prime}\left(x^{*}\right)$. Set for $x \in U^{\prime}\left(x^{*}\right)$

$$
\Phi_{1}(\lambda, x)=\prod_{\nu_{0}<\operatorname{Re} \lambda_{i}(x)<\nu}\left(\lambda+\lambda_{i}(x)\right) \prod_{\nu_{0} \leq n<\nu}(\lambda+n) .
$$

It follows from (3.10) that $\Phi_{1}(\lambda, x)(\mathcal{M} u)(\lambda, x)$ is holomorphic in $\{\lambda \in \mathbb{C} ; \operatorname{Re} \lambda>$ $-\nu-\epsilon\}$. Hence there exists $G_{1}(\lambda, x) \in \mathscr{O}\left(U^{\prime}\left(x^{*}\right)\right)[\lambda]$ with $\operatorname{deg} G_{1}(\lambda, x)<$ $\operatorname{deg} \Phi_{1}(\lambda, x)$ such that

$$
V_{1}(\lambda, x):=(\mathcal{M} u)(\lambda, x)-\frac{G_{1}(\lambda, x)}{\Phi_{1}(\lambda, x)}
$$


is holomorphic in $\{\lambda ; \operatorname{Re} \lambda>-\nu-\epsilon\} \times U^{\prime}\left(x^{*}\right)$. Let $0<t<a \leq 1$ and define

$$
\begin{aligned}
\tilde{u}(t, x) & =\frac{1}{2 \pi i} \int_{c-i \infty}^{c+i \infty} t^{-\lambda} \frac{(\mathcal{M} u)(\lambda, x)}{\lambda^{2}} d \lambda, \\
\tilde{v}_{1}(t, x) & =\frac{1}{2 \pi i} \int_{-\nu-i \infty}^{-\nu+i \infty} t^{-\lambda} \frac{V_{1}(\lambda, x)}{\lambda^{2}} d \lambda, \\
\tilde{w}_{1}(t, x) & =\frac{1}{2 \pi i} \int_{\mathcal{C}} t^{-\lambda} \frac{G_{1}(\lambda, x)}{\lambda^{2} \Phi_{1}(\lambda, x)} d \lambda, \\
w_{1}(t, x) & =\frac{1}{2 \pi i} \int_{\mathcal{C}} t^{-\lambda} \frac{G_{1}(\lambda, x)}{\Phi_{1}(\lambda, x)} d \lambda,
\end{aligned}
$$

where $-\hat{\nu}_{1}<-\nu<-\nu_{0}<c<0$ and $\mathcal{C}$ is a Jordan curve in $\{\operatorname{Re} \lambda<0\}$ enclosing all the zeros of $\Phi_{1}(\lambda, x)$ and $\lambda=0$ is outside of $\mathcal{C}$. Then by estimating $\tilde{u}(t, x), \tilde{v}_{1}(t, x)$ and $\tilde{w}_{1}(t, x)$, Lemma 1.2 and

$$
\frac{1}{2 \pi i} \int_{-\nu-i \infty}^{-\nu+\infty} t^{-\lambda} \frac{G_{1}(\lambda, x)}{\lambda^{2} \Phi_{1}(\lambda, x)} d \lambda=0 \quad \text { for } \quad 0<t<1,
$$

we have

$$
\left\{\begin{array}{l}
\tilde{u}(t, x)=\tilde{v}_{1}(t, x)+\tilde{w}_{1}(t, x), \\
\vartheta^{2} \tilde{u}(t, x)=u(t, x), \quad \vartheta^{2} \tilde{w}_{1}(t, x)=w_{1}(t, x), \\
|\tilde{u}(t, x)| \leq C|t|^{c},\left|\tilde{v}_{1}(t, x)\right| \leq C|t|^{\nu},\left|\tilde{w}_{1}(t, x)\right| \leq C|t|^{\nu_{0}} .
\end{array}\right.
$$

Put $v_{1}(t, x):=\vartheta^{2} \tilde{v}_{1}(t, x)=\vartheta^{2} \tilde{u}(t, x)-\vartheta^{2} \tilde{w}_{1}(t, x)=u(t, x)-w_{1}(t, x)$. Then

$$
\tilde{v}_{1}(t, x)=\int_{0}^{t} \frac{d t_{1}}{t_{1}} \int_{0}^{t_{1}}\left(u\left(t_{0}, x\right)-w_{1}\left(t_{0}, x\right)\right) \frac{d t_{0}}{t_{0}} .
$$

Since $u(t, x) \in \mathscr{O}\left(S \times U^{\prime}\right)$ and $w_{1}(t, x) \in \mathscr{M}_{\Phi_{1}}\left(U^{\prime}\left(x^{*}\right)\right), \tilde{v}_{1}(t, x), v_{1}(t, x) \in$ $\mathscr{O}\left(S \times U^{\prime}\left(x^{*}\right)\right)$. By applying Mellin transform $\mathcal{M}^{\phi}\left(|\phi|<\theta^{*}\right)$ to $u(t, x)=$ $v_{1}(t, x)+w_{1}(t, x)$, we can obtain the similar resuls, in particular, $\left(\mathcal{M}^{\phi} v_{1}\right)(\lambda, x)$ is holomorphic in $\{\operatorname{Re} \lambda>-\nu-\epsilon\}$. Hence for any $T \Subset S$ there exists a constant $C$ such that $\left|\tilde{v}_{1}(t, x)\right| \leq C|t|^{\nu}$ for $t \in T$ and $x \in U\left(x^{*}\right)$. Consequently there exist $r\left(x^{*}\right)>0$ and a constant $C_{0}=C_{0}\left(x^{*}, T\right)$ such that

$$
\left\{\begin{aligned}
& v_{1}(t, x)=\vartheta^{2} \tilde{v}_{1}(t, x) \underset{x^{*}}{\ll} C_{0}|t|^{\nu} \Theta\left(r: X-X^{*}\right) \\
& w_{1}(t, x) \underset{x^{*}}{\ll} C_{1}|t|^{\nu_{0}} \Theta\left(r: X-X^{*}\right) \quad \text { for } t \in T,
\end{aligned}\right.
$$

where $X-X^{*}=\sum_{i=1}^{d}\left(x_{i}-x_{i}^{*}\right)$. From $u(t, x)=v_{1}(t, x)+w_{1}(t, x)$ we have

$$
\left\{\begin{array}{l}
P(x, \vartheta) v_{1}(t, x)+Q_{1}\left(v_{1}\right)+f_{1}(t, x)=0, \\
Q_{1}\left(v_{1}\right)=Q\left(v_{1}+w_{1}\right)-Q\left(w_{1}\right), \\
f_{1}(t, x)=P(x, \vartheta) w_{1}(t, x)+Q\left(w_{1}\right)+f(t, x) .
\end{array}\right.
$$


Lemma 3.6. $\left(\mathcal{M} f_{1}\right)(\lambda, x)\left(x \in U^{\prime}\left(x^{*}\right)\right)$ is meromorphic in $\lambda$ on the whole plane $\mathbb{C}$ and its poles are in $\Lambda_{-\nu_{0}}(x)$.

Proof. By Lemma $3.2\left(\mathcal{M} w_{1}\right)(\lambda, x)$ is meromorphic and its poles are in $\left\{\Phi_{1}(\lambda, x)=0\right\}$. Hence $\left(\mathcal{M} P(x, \vartheta) w_{1}\right)(\lambda, x)$ and by Lemma $3.3\left(\mathcal{M} Q\left(w_{1}\right)\right)(\lambda, x)$ are meromorphic on the whole plane with poles in $\Lambda_{-\nu_{0}}(x)$, hence $\left(\mathcal{M} f_{1}\right)(\lambda, x)$ is so.

Lemma 3.7. $\left(\mathcal{M} Q_{1}\left(v_{1}\right)\right)(\lambda, x)\left(x \in U^{\prime}\left(x^{*}\right)\right)$ is holomorphic in $\{\lambda \in$ $\left.\mathbb{C} ; \operatorname{Re} \lambda>-\nu-\nu_{*}\right\}$ and $\left|\left(\mathcal{M} Q_{1}\left(v_{1}\right)\right)(\lambda, x)\right| \leq C a^{\operatorname{Re} \lambda} /\left(\operatorname{Re} \lambda+\nu+\nu_{*}\right)$ holds.

Proof. Let $T$ be a sector such that $T \Subset S$ and $t \in T$. Then it follows from (3.15) and Proposition 2.7 that there exists a constant $C_{1}$ such that

$$
\begin{aligned}
& q_{A}\left(v_{1}+w_{1}\right)-q_{A}\left(w_{1}\right) \\
= & t^{e_{A}} b_{A}(t, x) \sum_{\substack{\left(s_{\alpha}\right) \in N^{M *} \\
0 \leq s_{\alpha} \leq A_{\alpha}}}\left(\begin{array}{c}
A \\
s
\end{array}\right) \prod_{\alpha}\left(\vartheta^{\alpha_{0}} \partial_{z}^{\alpha^{\prime}} v_{1}\right)^{s_{\alpha}}\left(\vartheta^{\alpha_{0}} \partial_{z}^{\alpha^{\prime}} w_{1}\right)^{A_{\alpha}-s_{\alpha}} \\
\ll & \left.\underset{x^{*}}{ }\right|^{e_{A}+\nu_{0}(|A|-1)+\nu} C_{1}^{|A|} \Theta^{(m)}\left(r ; X-X^{*}\right) \\
= & |t|^{\nu+\nu_{*}+\left(e_{A}+\nu_{0}(|A|-1)-\nu_{*}\right)} C_{1}^{|A|} \Theta^{(m)}\left(r ; X-X^{*}\right) .
\end{aligned}
$$

If $t \in T$ with $|t|<t_{0}$ for a small $t_{0}>0$, by $e_{A}+\nu_{0}(|A|-1)-\nu_{*} \geq 0$ (see (3.5))

$$
Q_{1}\left(v_{1}\right)=\sum_{A}\left(q_{A}\left(v_{1}+w_{1}\right)-q_{A}\left(w_{1}\right)\right) \ll C_{2}|t|^{\nu+\nu_{*}} \Theta^{(m)}\left(r ; X-X^{*}\right) .
$$

Hence $\left(\mathcal{M} Q_{1}\left(v_{1}\right)\right)(\lambda, x)$ is holomorphic in $\left\{\lambda \in \mathbb{C} ; \operatorname{Re} \lambda>-\nu-\nu_{*}\right\}$. This implies the assertions.

Lemma 3.8. (1) $(\mathcal{M} u)(\lambda, x)$ is meromorphic in $\lambda$ on $\left\{\operatorname{Re} \lambda>-\hat{\nu}_{2}\right\}$ and its poles are contained in $\Lambda_{-\nu_{0}}(x)$.

(2) Let $\tilde{\nu}<\hat{\nu}_{2}$ and $L>0$ be a large constant such that $\{\lambda ; \operatorname{Re} \lambda>-\tilde{\nu},|\lambda| \geq$ $L\} \cap \Lambda_{-\nu_{0}}(x)=\emptyset$ for $x \in U^{\prime}\left(x^{*}\right)$. Then there exists a constant $C$ depending on $\tilde{\nu}$ such that for $\lambda \in\{\lambda ; \operatorname{Re} \lambda>-\tilde{\nu},|\lambda| \geq L\}$

$$
|(\mathcal{M} u)(\lambda, x)| \leq C a^{\operatorname{Re\lambda }} / \operatorname{Re} \lambda .
$$

Proof. We have from (3.16)

$$
\begin{aligned}
0 & =\left(\mathcal{M P} P(x, \vartheta) v_{1}\right)(x, \lambda)+\left(\mathcal{M} Q_{1}\left(v_{1}\right)\right)(x, \lambda)+\left(\mathcal{M} f_{1}\right)(x, \lambda) \\
& =P(x,-\lambda)\left(\mathcal{M} v_{1}\right)(x, \lambda)+a^{\lambda} H_{1}(x, \lambda)+\left(\mathcal{M} Q_{1}\left(v_{1}\right)\right)(x, \lambda)+\left(\mathcal{M} f_{1}\right)(x, \lambda) .
\end{aligned}
$$


Hence

$$
\left(\mathcal{M} v_{1}\right)(x, \lambda)=-\frac{a^{\lambda} H_{1}(x, \lambda)+\left(\mathcal{M} Q_{1}\left(v_{1}\right)\right)(x, \lambda)+\left(\mathcal{M} f_{1}\right)(x, \lambda)}{P(x,-\lambda)}
$$

and by Lemmas 3.6 and $3.7\left(\mathcal{M} v_{1}\right)(x, \lambda)\left(x \in U^{\prime}\left(x^{*}\right)\right)$ is meromorphic in $\left\{\operatorname{Re} \lambda>-\nu-\nu_{*}\right\}$ and its poles are in $\Lambda_{-\nu_{0}}(x)$. By $(\mathcal{M} u)(\lambda, x)=\left(\mathcal{M} v_{1}\right)(\lambda, x)+$ $\left(\mathcal{M} w_{1}\right)(\lambda, x),(\mathcal{M} u)(\lambda, x)$ is meromorphic in $\lambda$ in $\left\{\operatorname{Re} \lambda>-\nu-\nu_{*}\right\}$ and its poles are in $\Lambda_{-\nu_{0}}(x)$ for $x \in U^{\prime}\left(x^{*}\right)$. Since $\nu^{\prime}<\nu$ and $x^{*} \in U^{\prime}$ is arbitrary, this implies that $(\mathcal{M} u)(\lambda, x)\left(x \in U^{\prime}\right)$ is meromorphic in $\lambda$ on $\left\{\operatorname{Re} \lambda>-\nu^{\prime}-\nu_{*}\right\}$ and its poles are in $\Lambda_{-\nu_{0}}(x)$. Since $\nu^{\prime}$ is an arbitrary constant with $\nu^{\prime}<\nu_{1}=\nu_{0}+\nu_{*}$, $(\mathcal{M} u)(\lambda, x)$ is meromorphic in $\lambda$ in $\underset{\nu^{\prime}<\nu_{1}}{\cup}\left\{\operatorname{Re} \lambda>-\nu^{\prime}-\nu_{*}\right\}=\left\{\operatorname{Re} \lambda>-\hat{\nu}_{2}\right\}$. We have (3.17) from (3.18) and the estimate of $\left(\mathcal{M} Q_{1}\left(v_{1}\right)\right)(x, \lambda)$ in Lemma 3.7 .

Thus $(\mathcal{M} u)(\lambda, x)$ can be meromorphically extensible to $\left\{\operatorname{Re} \lambda>-\hat{\nu}_{2}\right\}$ and its poles are contained in $\Lambda_{-\nu_{0}}(x)$. Assume that $(\mathcal{M} u)(\lambda, x)$ is meromorphic in $\left\{\operatorname{Re} \lambda>-\hat{\nu}_{n}\right\}$ and its poles are contained in $\Lambda_{-\nu_{0}}(x)$. We repeat the same way as above. Let $x^{*} \in U^{\prime}$ and $\nu^{\prime}$ be an arbitrary number with $-\nu_{n}<-\nu^{\prime}<-\nu_{n-1}$. Then there exist a neighborhood $U^{\prime}\left(x^{*}\right)$ of $x^{*}$ and $\nu$ with $-\nu_{n}<-\nu<-\nu^{\prime}$ such that $(\mathcal{M} u)(\lambda, x)$ is holomorphic in $U^{\prime}\left(x^{*}\right) \times\{|\operatorname{Re} \lambda+\nu|<\epsilon\}$ for a small $\epsilon>0$. Hence there exist $G_{n}(\lambda, x), \Phi_{n}(\lambda, x) \in \mathscr{O}\left(U^{\prime}\left(x^{*}\right)\right)[\lambda]$ with $\operatorname{deg} G_{n}(\lambda, x)<$ $\operatorname{deg} \Phi_{n}(\lambda, x)$ such that the zeros of $\Phi_{n}(\lambda, x)$ are in $\{\operatorname{Re} \lambda \geq-\nu+\epsilon\} \cap \Lambda_{-\nu_{0}}(x)$ and

$$
V_{n}(\lambda, x):=(\mathcal{M} u)(\lambda, x)-\frac{G_{n}(\lambda, x)}{\Phi_{n}(\lambda, x)}
$$

is holomorphic in $\{\operatorname{Re} \lambda>-\nu-\epsilon\}$. Let $0<t<a \leq 1$ and define

$$
\begin{aligned}
\tilde{u}(t, x) & =\frac{1}{2 \pi i} \int_{c-i \infty}^{c+i \infty} t^{-\lambda} \frac{(\mathcal{M} u)(\lambda, x)}{\lambda^{2}} d \lambda, \\
\tilde{v}_{n}(t, x) & =\frac{1}{2 \pi i} \int_{-\nu-i \infty}^{-\nu+i \infty} t^{-\lambda} \frac{V_{n}(\lambda, x)}{\lambda^{2}} d \lambda, \\
\tilde{w}_{n}(t, x) & =\frac{1}{2 \pi i} \int_{\mathcal{C}} t^{-\lambda} \frac{G_{n}(\lambda, x)}{\lambda^{2} \Phi_{n}(\lambda, x)} d \lambda, \\
w_{n}(t, x) & =\frac{1}{2 \pi i} \int_{\mathcal{C}} t^{-\lambda} \frac{G_{n}(\lambda, x)}{\Phi_{n}(\lambda, x)} d \lambda,
\end{aligned}
$$

where $-\hat{\nu}_{n}<-\nu<-\nu^{\prime}<-\hat{\nu}_{n-1}<-\nu_{0}<c<0$ and $\mathcal{C}$ is a Jordan curve in 
$\{\operatorname{Re} \lambda<0\}$ enclosing all the zeros of $\Phi_{n}(\lambda, x)$. Then

$$
\left\{\begin{array}{l}
\tilde{u}(t, x)=\tilde{v}_{n}(t, x)+\tilde{w}_{n}(t, x), \\
\vartheta^{2} \tilde{u}(t, x)=u(t, x), \quad \vartheta^{2} \tilde{w}_{n}(t, x)=w_{n}(t, x), \\
|\tilde{u}(t, x)| \leq C|t|^{c},\left|\tilde{v}_{n}(t, x)\right| \leq C|t|^{\nu},\left|\tilde{w}_{n}(t, x)\right| \leq C|t|^{\nu_{0}}
\end{array}\right.
$$

Define $v_{n}(t, x):=\vartheta^{2} \tilde{v}_{n}(t, x)=\vartheta^{2} \tilde{u}(t, x)-\vartheta^{2} \tilde{w}_{n}(t, x)=u(t, x)-w_{n}(t, x)$. Then $w_{n}(t, x) \in \mathscr{M}_{\Phi_{n}}\left(U^{\prime}\left(x^{*}\right)\right)$ and $v_{n}(t, x) \in \mathscr{O}\left(S \times U^{\prime}\left(x^{*}\right)\right)$. We can show that for any $T \Subset S$ there exists a constant $C$ such that $\left|\tilde{v}_{n}(t, x)\right| \leq C|t|^{\nu}$ for $t \in T$ and $x \in U\left(x^{*}\right)$. Consequently there exist $r=r\left(x^{*}\right)>0$ and $C_{0}=C_{0}\left(x^{*}, \nu, T\right)$ such that

$$
\left\{\begin{array}{c}
v_{n}(t, x)=\vartheta^{2} \tilde{v}_{n}(t, x) \underset{x^{*}}{\ll} C_{0}|t|^{\nu} \Theta\left(r: X-X^{*}\right) \\
w_{n}(t, x) \underset{x^{*}}{\ll} C_{0}|t|^{\nu_{0}} \Theta\left(r: X-X^{*}\right) \quad \text { for } t \in T,
\end{array}\right.
$$

where $X-X^{*}=\sum_{i=1}^{d}\left(x_{i}-x_{i}^{*}\right)$. Set

$$
\left\{\begin{array}{l}
Q_{n}\left(v_{n}\right):=Q\left(v_{n}+w_{n}\right)-Q\left(w_{n}\right), \\
f_{n}(t, x):=P(x, \vartheta) w_{n}+Q\left(w_{n}\right)+f(t, x) .
\end{array}\right.
$$

Then

$$
P(x, \vartheta) v_{n}+Q_{n}\left(v_{n}\right)+f_{n}(t, x)=0 .
$$

By repeating the preceding arguments in Lemmas 3.6 and 3.7, it follows from (3.22) and (3.23) that

1. $\left(\mathcal{M} f_{n}\right)(\lambda, x)\left(x \in U\left(x^{*}\right)\right)$ is meromorphic in $\lambda$ on the whole plane $\mathbb{C}$ and its poles are in $\Lambda_{-\nu_{0}}(x)$

and

2. for any $T \Subset S$ there exists a constant $C=C\left(x^{*}, \nu, T\right)$ such that

$$
Q_{n}\left(v_{n}\right) \underset{x^{*}}{\ll} C|t|^{\nu+\nu_{*}} \Theta^{(m)}\left(r ; X-X^{*}\right) \quad \text { for } x \in U^{\prime}\left(x^{*}\right) \text { and } t \in T,
$$

hence $\left(\mathcal{M} Q_{n}\left(v_{n}\right)\right)(\lambda, x)$ is holomorphic in $\left\{\operatorname{Re} \lambda>-\nu-\nu_{*}\right\}$.

By applying Mellin transform to (3.24),

$$
\left(\mathcal{M} v_{n}\right)(x, \lambda)=-\frac{a^{\lambda} H_{n}(x, \lambda)+\left(\mathcal{M} Q_{n}\left(v_{n}\right)\right)(x, \lambda)+\left(\mathcal{M} f_{n}\right)(x, \lambda)}{P(x,-\lambda)},
$$


where $H_{n}(x, \lambda)$ is a polynomial. It follows from $(3.26)$ that $\left(\mathcal{M} v_{n}\right)(x, \lambda)(x \in$ $\left.U^{\prime}\left(x^{*}\right)\right)$ is meromorphically extensible to $\left\{\operatorname{Re} \lambda>-\nu-\nu_{*}\right\}$ and its poles are in $\Lambda_{-\nu_{0}}(x)$, hence by $(\mathcal{M} u)(\lambda, x)=\left(\mathcal{M} v_{n}\right)(\lambda, x)+\left(\mathcal{M} w_{n}\right)(\lambda, x),(\mathcal{M} u)(\lambda, x)$ is meromorphic in $\left\{\operatorname{Re} \lambda>-\nu-\nu_{*}\right\}$ and its poles are in $\Lambda_{-\nu_{0}}(x)$ for $x \in U^{\prime}\left(x^{*}\right)$. Consequently $(\mathcal{M} u)(\lambda, x)$ is meromorphic in $\left\{\operatorname{Re} \lambda>-\nu^{\prime}-\nu_{*}\right\}$ and its poles are in $\Lambda_{-\nu_{0}}(x)$ for $x \in U^{\prime}$. We can take $\nu^{\prime}<\hat{\nu_{n}}$ as close to $\hat{\nu}_{n}$ as possible, hence $(\mathcal{M} u)(\lambda, x)$ is meromorphic in $\left\{\operatorname{Re} \lambda>-\nu_{n+1}\right\}$. Thus by induction we concude that $(\mathcal{M} u)(\lambda, x)$ is meromorphic on the whole plane and its poles are contained in $\Lambda_{-\nu_{0}}(x)$ and have Theorem 1.3.

Lemmas 3.1, 3.2 and 1.2 remain unproven, so we prove them.

Proof of Lemma 3.1. Let

$$
w_{i}(t, x)=\frac{1}{2 \pi i} \int_{\mathcal{C}_{i}} t^{-\lambda} \frac{\psi_{i}(\lambda, x)}{\varphi_{i}(\lambda, x)} d \lambda
$$

where $\varphi_{i}(\lambda, x)=a_{i}(x) \prod_{j=1}^{p_{i}}\left(\lambda-a_{i}^{j}(x)\right) \in \mathscr{O}\left(U^{\prime}\right)[\lambda](i=1,2)$ and $a_{i}(x) \neq 0$ in $U^{\prime}$. We may assume $a_{1}(x)=a_{2}(x)=1$. We have

$$
\begin{aligned}
w_{1}(t, x) w_{2}(t, x) & =\frac{1}{(2 \pi i)^{2}} \int_{\mathcal{C}_{1}} t^{-\lambda} \frac{\psi_{1}(\lambda, x)}{\varphi_{1}(\lambda, x)} d \lambda \int_{\mathcal{C}_{2}} t^{-\mu} \frac{\psi_{2}(\mu, x)}{\varphi_{2}(\mu, x)} d \mu \\
& =\frac{1}{(2 \pi i)^{2}} \int_{\mathcal{C}_{1}} \int_{\mathcal{C}_{2}} t^{-(\lambda+\mu)} \frac{\psi_{1}(\lambda, x) \psi_{2}(\mu, x)}{\varphi_{1}(\lambda, x) \varphi_{2}(\mu, x)} d \lambda d \mu \\
& =\frac{1}{2 \pi i} \int_{\mathcal{C}} t^{-\rho} \Phi(\rho, x) d \rho
\end{aligned}
$$

where

$$
\Phi(\rho, x)=\frac{1}{2 \pi i} \int_{\mathcal{C}_{2}} \frac{\psi_{1}(\rho-\mu, x) \psi_{2}(\mu, x)}{\varphi_{1}(\rho-\mu, x) \varphi_{2}(\mu, x)} d \mu
$$

and $\mathcal{C}$ is a Jordan curve such that the interior of $\mathcal{C}$ contains the disk $\{|\rho| \leq r\}$ for a large $r$. Put

$$
\left\{\begin{array}{l}
\phi(\rho, \mu, x)=\prod_{j=1}^{p_{2}}\left(1-\frac{\varphi_{1}(\rho-\mu, x)}{\varphi_{1}\left(\rho-a_{2}^{j}(x), x\right)}\right), \\
\psi(\rho, \mu, x)=1-\phi(\rho, \mu, x) .
\end{array}\right.
$$

Then we have

$$
\begin{aligned}
\Phi(\rho, x)= & \frac{1}{2 \pi i} \int_{\mathcal{C}_{2}} \frac{\psi_{1}(\rho-\mu, x) \psi_{2}(\mu, x) \phi(\rho, \mu, x)}{\varphi_{1}(\rho-\mu, x) \varphi_{2}(\mu, x)} d \mu \\
& +\frac{1}{2 \pi i} \int_{\mathcal{C}_{2}} \frac{\psi_{1}(\rho-\mu, x) \psi_{2}(\mu, x) \psi(\rho, \mu, x)}{\varphi_{1}(\rho-\mu, x) \varphi_{2}(\mu, x)} d \mu \\
= & \Phi_{1}(\rho, x)+\Phi_{2}(\rho, x) .
\end{aligned}
$$


It follows from $\left.\phi(\rho, \mu, x)\right|_{\mu=a_{2}^{j}(x)}=0$ for all $1 \leq j \leq p_{2}$ that $\frac{\phi(\rho, \mu, x)}{\varphi_{2}(\mu, x)}$ is holomorphic in $\mu$, so $\Phi_{1}(\rho, x)=0$. We have

$$
\begin{aligned}
& \left(\varphi_{1} \# \varphi_{2}\right)(\rho, x) \psi(\rho, \mu, x) \\
= & \prod_{j=1}^{p_{2}} \varphi_{1}\left(\rho-a_{2}^{j}(x), x\right)-\prod_{j=1}^{p_{2}}\left(\varphi_{1}\left(\rho-a_{2}^{j}(x), x\right)-\varphi_{1}(\rho-\mu, x)\right)
\end{aligned}
$$

and it is divisible by $\varphi_{1}(\rho-\mu, x)$ and

$$
\frac{\psi_{1}(\rho-\mu, x)\left(\varphi_{1} \# \varphi_{2}\right)(\rho, x) \psi(\rho, \mu, x)}{\varphi_{1}(\rho-\mu, x)}
$$

is a polynomial in $\rho$ with degree $\leq p_{1}-1+p_{1} p_{2}-p_{1}=p_{1} p_{2}-1$. Thus

$$
\begin{aligned}
\Psi_{2}(\rho, x) & :=\left(\varphi_{1} \# \varphi_{2}\right)(\rho, x) \Phi_{2}(\rho, x) \\
& =\frac{1}{2 \pi i} \int_{\mathcal{C}_{2}} \frac{\psi_{1}(\rho-\mu, x)\left(\varphi_{1} \# \varphi_{2}\right)(\rho, x) \psi(\rho, \mu, x)}{\varphi_{1}(\rho-\mu, x)} \frac{\psi_{2}(\mu, x)}{\varphi_{2}(\mu, x)} d \mu
\end{aligned}
$$

is a polynomial in $\rho$ with $\operatorname{deg} \Psi_{2}(\rho, x) \leq p_{1} p_{2}-1$ and we have

$$
\Phi(\rho, x)=\Phi_{2}(\rho, x)=\frac{\Psi_{2}(\rho, x)}{\left(\varphi_{1} \# \varphi_{2}\right)(\rho, x)} .
$$

Proof of Lemma 3.2. Let

$$
w(t, x)=\frac{1}{2 \pi i} \int_{\mathcal{C}} t^{-\mu} \frac{\psi(\mu, x)}{\varphi(\mu, x)} d \mu .
$$

Then for $\lambda$ with large real part

$$
\begin{aligned}
(\mathcal{M} w)(\lambda, x) & =\int_{0}^{a} t^{\lambda-1} w(t, x) d t=\frac{1}{2 \pi i} \int_{0}^{a} t^{\lambda-1} d t \int_{\mathcal{C}} t^{-\mu} \frac{\psi(\mu, x)}{\varphi(\mu, x)} d \mu \\
& =\frac{1}{2 \pi i} \int_{\mathcal{C}} \frac{a^{\lambda-\mu}}{(\lambda-\mu)} \frac{\psi(\mu, x)}{\varphi(\mu, x)} d \mu .
\end{aligned}
$$

Let $C_{\lambda}$ be a small circle with center $\lambda$ and $\hat{C}$ be a Jordan curve such that $\{\mu ;(\mu-\lambda) \varphi(\mu, x)=0\}$ is in its interior. Then

$$
\begin{aligned}
(\mathcal{M} w)(\lambda, x) & =\frac{1}{2 \pi i} \int_{\hat{\mathcal{C}}} \frac{a^{\lambda-\mu}}{(\lambda-\mu)} \frac{\psi(\mu, x)}{\varphi(\mu, x)} d \mu-\frac{1}{2 \pi i} \int_{\mathcal{C}_{\lambda}} \frac{a^{\lambda-\mu}}{(\lambda-\mu)} \frac{\psi(\mu, x)}{\varphi(\mu, x)} d \mu \\
& =\frac{\psi(\lambda, x)}{\varphi(\lambda, x)}+\frac{1}{2 \pi i} \int_{\hat{\mathcal{C}}} \frac{a^{\lambda-\mu}}{(\lambda-\mu)} \frac{\psi(\mu, x)}{\varphi(\mu, x)} d \mu=\frac{\psi(\lambda, x)}{\varphi(\lambda, x)}+\phi(\lambda, x),
\end{aligned}
$$


where $\phi(\lambda, x)$ is an entire function in $\lambda$.

Proof of Lemma 1.2. Let $\psi(t)$ be a solution of $\vartheta^{s} \psi(t)=f(t)$ such that $|\psi(t)| \leq C|t|^{\kappa_{0}}$, which is given by

$$
\psi(t)=\int_{0}^{t} \frac{d t_{s-1}}{t_{s-1}} \int_{0}^{t_{s-1}} \frac{d t_{s-2}}{t_{s-2}} \cdots \int_{0}^{t_{2}} \frac{d t_{1}}{t_{1}} \int_{0}^{t_{1}} \frac{f\left(t_{0}\right) d t_{0}}{t_{0}} .
$$

We have from lemma 3.3

$$
(-\lambda)^{s}(\mathcal{M} \psi)(\lambda)=(\mathcal{M} f)(\lambda)+a^{\lambda} H(\lambda),
$$

where $H(\lambda)$ is a polynomial with degree $<s$. By the inverse Mellin transform for $0<t<a$

$$
\begin{aligned}
\psi(t) & =\lim _{N \rightarrow \infty} \frac{1}{2 \pi i} \int_{c-i N}^{c+i N} t^{-\lambda}(\mathcal{M} \psi)(\lambda) d \lambda \quad\left(-\kappa_{0}<c<0\right) \\
& =\lim _{N \rightarrow \infty} \frac{1}{2 \pi i} \int_{c-i N}^{c+i N} t^{-\lambda} \frac{(\mathcal{M} f)(\lambda)+a^{\lambda} H(\lambda)}{(-\lambda)^{s}} d \lambda .
\end{aligned}
$$

Let us show

$$
\lim _{N \rightarrow \infty} \frac{1}{2 \pi i} \int_{c-i N}^{c+i N} t^{-\lambda} \frac{a^{\lambda} H(\lambda)}{(-\lambda)^{s}} d \lambda=0
$$

In order to do so we show $\lim _{N \rightarrow \infty} \int_{c-i N}^{c+i N} \frac{t^{-\lambda} a^{\lambda}}{\lambda^{\ell}} d \lambda=0$ for $\ell \geq 1$. We have (3.29)

$$
\int_{c-i N}^{c+i N} \frac{t^{-\lambda} a^{\lambda}}{\lambda^{\ell}} d \lambda=i\left(\frac{a}{t}\right)^{c} \int_{-N}^{+N} \frac{(a / t)^{i x}}{(c+i x)^{\ell}} d x=i^{1-\ell}\left(\frac{a}{t}\right)^{c} \int_{-N}^{+N} \frac{(a / t)^{i x}}{(x-i c)^{\ell}} d x .
$$

Since $c<0$, by Cauchy's integral theorem

$$
\int_{-N}^{+N} \frac{(a / t)^{i x}}{(x-i c)^{\ell}} d x+\int_{\mathcal{C}_{N}} \frac{(a / t)^{i z}}{(z-i c)^{\ell}} d z=0 \quad(z=x+i y),
$$

where $\mathcal{C}_{N}$ is a path defined by $z=N e^{i \theta}(0 \leq \theta \leq \pi)$. From

$$
\int_{\mathcal{C}_{N}} \frac{(a / t)^{i z}}{(z-i c)^{\ell}} d z=i N \int_{0}^{\pi} \frac{(a / t)^{i N e^{i \theta}} e^{i \theta}}{\left(N e^{i \theta}-i c\right)^{\ell}} d \theta
$$

we have

$$
\left|\int_{\mathcal{C}_{N}} \frac{(a / t)^{i z}}{(z-i c)^{\ell}} d z\right| \leq N \int_{0}^{\pi} \frac{(a / t)^{-N \sin \theta}}{\left.\mid N e^{i \theta}-i c\right)\left.\right|^{\ell}} d \theta .
$$

If $\frac{a}{t}>1$ and $\ell \geq 1$, we have $\lim _{N \rightarrow \infty} \int_{\mathcal{C}_{N}} \frac{(a / t)^{i z}}{(z-i c)^{\ell}} d z=0$. It follows from (3.29) and (3.30) that $\lim _{N \rightarrow \infty} \int_{c-i N}^{c+i N} \frac{t^{-\lambda} a^{\lambda}}{\lambda^{\ell}} d \lambda=0$. Hence we have (3.28) and

$$
\psi(t)=\lim _{N \rightarrow \infty} \frac{1}{2 \pi i} \int_{c-i N}^{c+i N} t^{-\lambda} \frac{(\mathcal{M} f)(\lambda)}{(-\lambda)^{s}} d \lambda \quad(0<t<a) .
$$


Thus $\psi(t)=\psi^{*}(t)$ and $\vartheta^{s} \psi^{*}(t)=f(t)$.

\section{§4. Nonlinear Equations with Singular Coefficients}

In order to show Theorem 1.5 we transform the original equation $L(u)=0$ to one with coefficients singular at $t=0$ and study it instead of the original $L(u)=0$. Let $L(u)$ be a nonlinear partial differential operator with (1.6) that satisfies (1.7) and $u(t, x) \in \mathscr{O}\left(S \times U^{\prime}\right)$ be a solution of $L(u)=0$ with bound (1.8). In the following a polydisk $U^{\prime}=\left\{x \in \mathbb{C}^{d} ;|x|<R^{\prime}\right\}$ is small, if necessary. Let

$$
\lambda^{*}=\max _{1 \leq i \leq k} \sup _{x \in U^{\prime}} \operatorname{Re} \lambda_{i}(x) .
$$

Choose $\nu \geq \nu_{0}$ such that $\nu>\max \left\{\lambda^{*}, 1\right\}$ and $\{\operatorname{Re} \lambda=-\nu\} \cap \Lambda(x)=\emptyset$ for $x \in U^{\prime}$.

First we decompose $u(t, x)$. As in Section 3 we can find $w(t, x) \in \mathscr{M}_{\phi}\left(W^{\prime}\right)$, where $W^{\prime} \subset U^{\prime}$ is a polydisk centered at $x=0$ and $\{\lambda ; \phi(\lambda, x)=0\} \subset \Lambda_{-\nu_{0}}(x) \cap$ $\{\lambda ; \operatorname{Re} \lambda>-\nu\}$ for $x \in W^{\prime}$, such that

$$
\left\{\begin{array}{l}
w(t, x) \ll C|t|^{\nu_{0}} \Theta(r ; X), X=\sum_{i=1}^{d} x_{i}, \\
v(t, x):=u(t, x)-w(t, x) \ll C|t|^{\nu} \Theta(r ; X) .
\end{array}\right.
$$

By substituting $u(t, x)=v(t, x)+w(t, x)$ for $L(u)=0$, we have a new equation $L^{w}(v):=L(v+w)=0$ with unknown $v$. By setting

$$
\left\{\begin{array}{l}
Q^{w}(v):=Q(v+w)-Q(w), \\
f^{w}(t, x):=P(x, \vartheta) w(t, x)+Q(w)+f(t, x),
\end{array}\right.
$$

we have

$$
L^{w}(v)=P\left(x, \vartheta_{t}\right) v(t, x)+Q^{w}(v)+f^{w}(t, x)=0,
$$

where

$$
\begin{aligned}
Q^{w}(v) & =\sum_{B \in \mathbb{N}^{M^{*}}} c_{B}(t, x) \\
& \times\left(\sum_{\substack{\left(s_{\alpha}\right) \in N^{M *} \\
0 \leq s_{\alpha} \leq B_{\alpha}}}\left(\begin{array}{c}
B \\
s
\end{array}\right) \prod_{\alpha}\left(\vartheta^{\alpha_{0}} \partial_{z}^{\alpha^{\prime}} v\right)^{s_{\alpha}}\left(\vartheta^{\alpha_{0}} \partial_{z}^{\alpha^{\prime}} w\right)^{B_{\alpha}-s_{\alpha}}\right) .
\end{aligned}
$$


We represent $Q^{w}(v)$ in another form

$$
Q^{w}(v)=\sum_{A \in \mathbb{N}^{*}} c_{A}^{w}(t, x) \prod_{A}\left(\vartheta^{\alpha_{0}} \partial^{\alpha^{\prime}} v(t, x)\right)^{A_{\alpha}},
$$

where

$$
c_{A}^{w}(t, x)=\sum_{\substack{B \in \mathbb{N}^{M *} \\
B \geq A}}\left(\begin{array}{l}
B \\
A
\end{array}\right) c_{B}(t, x) \prod_{\alpha}\left(\vartheta^{\alpha_{0}} \partial^{\alpha^{\prime}} w(t, x)\right)^{B_{\alpha}-A_{\alpha}} .
$$

Set

$$
e_{A}^{w}=\min _{\{B ; B \geq A\}}\left\{e_{B}+\nu_{0}(|B|-|A|)\right\} .
$$

Then $e_{A}^{w} \leq e_{A}$ and we have

Lemma 4.1. (1) $e_{A}^{w} \geq \min \left\{1, \nu_{0}\right\}$ for $A \in \mathbb{N}^{M *}$ with $|A|=1$.

(2) There exists a constant $C$ such that

$$
f^{w}(t, x) \ll C|t|^{\nu} \Theta(r ; X), X=\sum_{i=1}^{d} x_{i} .
$$

$$
\frac{e_{A}^{w}+\nu_{0}(|A|-1)}{\gamma} \geq\left(m_{A}-k\right)
$$

Proof. (1) Let $|A|=1$. Then $e_{A}^{w}=\min _{\{B ; B \geq A\}}\left\{e_{B}+\nu_{0}(|B|-1)\right\}$. If $|B|=$ $1, e_{B} \geq 1$ and if $|B| \geq 2, e_{B}+\nu_{0}(|B|-1) \geq \nu_{0}$. So we have $e_{A}^{w} \geq \min \left\{1, \nu_{0}\right\}$. (2) We have $f^{w}(t, x)=P\left(x, \vartheta_{t}\right) w(t, x)+Q(w)+f(t, x)=-P\left(x, \vartheta_{t}\right) v(t, x)-$ $(Q(v+w)-Q(w))$. It follows from (4.2) and Propositin 2.5 that $P\left(x, \vartheta_{t}\right) v(t, x)$, $Q(v+w)-Q(w) \ll C_{1}|t|^{\nu} \Theta(r ; X)$, hence we have (4.7).

(3) There exists $B \in \mathbb{N}^{M *}$ with $B \geq A$ such that $e_{A}^{w}=e_{B}+\nu_{0}(|B|-|A|)$, and obviously $m_{B} \geq m_{A}$. Hence $e_{A}^{w}+\nu_{0}(|A|-1)=e_{B}+\nu_{0}(|B|-1) \geq \gamma\left(m_{B}-k\right) \geq$ $\gamma\left(m_{A}-k\right)$ by the definition of the index $\gamma$.

Let us decompose coefficients $c_{A}^{w}(t, x)$ of $Q^{w}(v)$ and $f^{w}(t, x)$ to the sum of functions in $\mathscr{M}_{\text {rat }}\left(U^{\prime}\right)$. Before doing so, let us introduce a subspace $\mathscr{M}_{\left\{\Lambda_{-\nu_{0}}(x)\right\}}\left(U^{\prime}\right)$ of $\mathscr{M}_{\text {rat }}\left(U^{\prime}\right)$.

Definition 4.2. $\mathscr{M}_{\left\{\Lambda_{-\nu_{0}}(x)\right\}}\left(U^{\prime}\right)$ is the totality of $f(t, x) \in \mathscr{M}_{\varphi}\left(U^{\prime}\right)$ such that $\{\lambda ; \varphi(\lambda, x)=0\} \subset\left\{\Lambda_{-\nu_{0}}(x)\right\}$. 
By Taylor expansion of $c_{B}(t, x)$, we have

$$
\begin{aligned}
c_{A}^{w}(t, x) & =\sum_{\substack{B \in \mathbb{N}^{M *} \\
B \geq A}}\left(\begin{array}{l}
B \\
A
\end{array}\right) \sum_{\ell=e_{B}}^{\infty} c_{B, \ell}(x) t^{\ell} \prod_{\alpha}\left(\vartheta^{\alpha_{0}} \partial^{\alpha^{\prime}} w(t, x)\right)^{B_{\alpha}-A_{\alpha}} \\
& =\sum_{n=0}^{\infty} c_{A, n}^{w}(t, x),
\end{aligned}
$$

where

(4.10)

$$
c_{A, n}^{w}(t, x)=\sum_{\substack{\ell \in \mathbb{N}, B \in \mathbb{N}^{M *} ; \ell \geq e_{B}, B \geq A \\
\left[\nu_{0}(|B|-|A|)+\ell-e_{A}^{w}\right]=n}}\left(\begin{array}{c}
B \\
A
\end{array}\right) c_{B, \ell}(x) t^{\ell} \prod_{\alpha}\left(\vartheta^{\alpha_{0}} \partial^{\alpha^{\prime}} w(t, x)\right)^{B_{\alpha}-A_{\alpha}},
$$

which is in $\mathscr{M}_{\left\{\Lambda_{-\nu_{0}}(x)\right\}}\left(U^{\prime}\right)$. Next we decompose $f^{w}(t, x)=P(x, \vartheta) w(t, x)+$ $Q(w)+f(t, x)$. We have

$$
\begin{aligned}
Q(w)= & \sum_{A \in \mathbb{N}^{M^{*}}} c_{A}(t, x) \prod_{\alpha}\left(\vartheta^{\alpha_{0}} \partial_{z}^{\alpha^{\prime}} w\right)^{A_{\alpha}} \\
= & \sum_{A \in \mathbb{N}^{M^{*}}} \sum_{\ell=e_{A}}^{+\infty} c_{A, \ell}(x) t^{\ell} \prod_{\alpha}\left(\vartheta^{\alpha_{0}} \partial_{z}^{\alpha^{\prime}} w\right)^{A_{\alpha}} \\
= & \left(\sum_{\left\{\sum_{A, \ell} c_{A, \mathbb{N}, A \in \mathbb{N}^{*} ; \ell \geq e_{A}}\right\}} c_{A, \ell}(x) t^{\ell} \prod_{\alpha}\left(\vartheta^{\alpha_{0}} \partial_{z}^{\alpha^{\prime}} w\right)^{A_{\alpha}}\right) \\
& +\sum_{n=1}^{\infty}\left(\sum_{\substack{\ell \in \mathbb{N}, A \in \mathbb{N}^{M *} ; \ell \geq e_{A} \\
\left[\nu_{0}|A|+\ell-\nu\right]=n}} c_{A, \ell}(x) t^{\ell} \prod_{\alpha}\left(\vartheta^{\alpha_{0}} \partial_{z}^{\alpha^{\prime}} w\right)^{A_{\alpha}}\right)
\end{aligned}
$$

and $f(t, x)=\sum_{\ell \in \mathbb{N} ; \ell \geq \nu_{0}} f_{\ell}(x) t^{\ell}$. Define $\left\{f_{n}^{w}(t, x)\right\}_{n \in \mathbb{N}} \subset \mathcal{M}_{\left\{\Lambda_{-\nu_{0}}(x)\right\}}(U)$ by

$$
\begin{aligned}
f_{0}^{w}(t, x):= & \left.\sum_{\substack{\left.\ell \in \mathbb{N}, A \in \mathbb{N}^{M *} ; \ell \geq e_{A}\right\} \\
\nu_{0}|A|+\ell-\nu<1}} c_{A, \ell}(x) t^{\ell} \prod_{\alpha}\left(\vartheta^{\alpha_{0}} \partial_{z}^{\alpha^{\prime}} w\right)^{A_{\alpha}}\right) \\
+ & P\left(x, \vartheta_{t}\right) w(t, x)+\sum_{\ell<1+\nu} f_{\ell}(x), \\
f_{n}^{w}(t, x):= & \left.\sum_{\substack{\ell \in \mathbb{N}, A \in \mathbb{N}^{M *} ; \ell \geq e_{A} \\
\left[\nu_{0}|A|+\ell-\nu\right]=n}} c_{A, \ell}(x) t^{\ell} \prod_{\alpha}\left(\vartheta^{\alpha_{0}} \partial_{z}^{\alpha^{\prime}} w\right)^{A_{\alpha}}\right) \\
+ & f_{n_{\nu}}(x),
\end{aligned}
$$


where $n_{\nu} \in \mathbb{N}$ with $n+\nu \leq n_{\nu}<n+1+\nu$. Thus we have

$$
f^{w}(t, x)=\sum_{n=0}^{\infty} f_{n}^{w}(t, x) .
$$

Proposition 4.3. (1) Let $r_{0}, r^{\prime}>0$ be small constants and $\theta^{\prime}>0$. Then there exist $C_{0}$ and $C_{1}$ such that

$$
\sup _{|\arg t|<\theta^{\prime}, 0<|t|<r_{0},|x|<r^{\prime}}|t|^{-n-e_{A}^{w}}\left|c_{A, n}^{w}(t, x)\right| \leq C_{0}^{|A|} C_{1}^{n+e_{A}^{w}}
$$

and

$$
\sup _{|\arg t|<\theta^{\prime}, 0<|t|<r_{0},|x|<r^{\prime}}|t|^{-n-\nu}\left|f_{n}^{w}(t, x)\right| \leq C_{0} C_{1}^{n}
$$

(2) The poles of $\left(\mathcal{M} c_{A, n}^{w}\right)(\lambda, x)$ are in $\left\{-\frac{\nu}{\nu_{0}}\left(n+1+e_{A}^{w}\right)<\operatorname{Re} \lambda \leq-n-\right.$ $\left.e_{A}^{w}\right\} \cap \Lambda_{-\nu_{0}}(x)$ and those of $\left(\mathcal{M} f_{n}^{w}\right)(\lambda, x)$ are in $\left\{-\frac{\nu}{\nu_{0}}(n+1+\nu)<\operatorname{Re} \lambda \leq\right.$ $-n-\nu\} \cap \Lambda_{-\nu_{0}}(x)$.

Proof. (1) It follows from $\left|c_{B, \ell}(x)\right| \leq C_{0}^{|B|} C_{1}^{\ell}$ and $\prod_{\alpha}\left|\vartheta^{\alpha_{0}} \partial^{\alpha^{\prime}} w(t, x)\right|^{B_{\alpha}-A_{\alpha}}$ $\leq C_{0}^{(|B|-|A|)}|t|^{\nu_{0}(|B|-|A|)}$ in a small neighborhood of $x=0$ that there exists a constnat $C_{2}$ such that

$$
\begin{aligned}
& |t|^{-e_{A}^{w}}\left|c_{A, n}^{w}(t, x)\right| \\
& \leq \sum_{\substack{\ell \in \mathbb{N}, B \in \mathbb{N}^{M *} ; \ell \geq e_{B}, B \geq A \\
\left[\nu_{0}(|B|-|A|)+\ell-e_{A}^{w}\right]=n}}\left(\begin{array}{c}
B \\
A
\end{array}\right)\left|c_{B, \ell}(x)\right||t|^{\ell-e_{A}^{w}} \prod_{\alpha}\left|\vartheta^{\alpha_{0}} \partial^{\alpha^{\prime}} w(t, x)\right|^{B_{\alpha}-A_{\alpha}} \\
& \leq C_{0}^{-|A|}\left(\sum_{\substack{\left\{\in \mathbb{N}, B \in \mathbb{N}^{M *} ; \ell \geq e_{B}, B \geq A \\
\left[\nu_{0}(|B|-|A|)+\ell-e_{A}^{w}\right]=n\right.}}\left(\begin{array}{c}
B \\
A
\end{array}\right) C_{0}^{2|B|} C_{1}^{\ell}|t|^{\nu_{0}(|B|-|A|)+\ell-e_{A}^{w}}\right) \\
& \leq C_{0}^{-|A|} C_{1}^{e_{A}^{w}}\left(\sum_{\substack{\left\{\in \mathbb{N}, B \in \mathbb{N}^{M *} ; \ell \geq e_{B}, B \geq A \\
\left[\nu_{0}(|B|-|A|)+\ell-e_{A}^{w}\right]=n\right.}}\left(\begin{array}{c}
B \\
A
\end{array}\right) C_{0}^{2|B|} C_{1}^{\ell-e_{A}^{w}}\right)|t|^{n} \\
& \leq C_{0}^{-|A|} C_{1}^{e_{A}^{w}} C_{2}^{n+|A|+1}|t|^{n} .
\end{aligned}
$$

By choosing other $C_{0}$ and $C_{1},(4.13)$ holds. We also have (4.14) by the similar method.

(2) From the definition of $w(t, x)$, the poles of $\left(\mathcal{M} t^{\ell} \prod_{\alpha}\left(\vartheta^{\alpha_{0}} \partial^{\alpha^{\prime}} w\right)^{B_{\alpha}-A_{\alpha}}\right)(\lambda, x)$ 
are in $\left\{-\ell-\nu(|B|-|A|)<\operatorname{Re} \lambda \leq-\ell-\nu_{0}(|B|-|A|)\right\}$. Let $n+e_{A}^{w} \leq \ell+\nu_{0}(|B|-$ $|A|)<n+1+e_{A}^{w}$. Then we have $(|B|-|A|)<\left(n+1+e_{A}^{w}\right) / \nu_{0}$ and

$$
-\ell-\nu(|B|-|A|)>\left(\nu_{0}-\nu\right)(|B|-|A|)-\left(n+1+e_{A}^{w}\right)>-\frac{\nu}{\nu_{0}}\left(n+1+e_{A}^{w}\right) .
$$

Hence from the definition of $c_{A, n}^{w}(t, x)$ and (4.13) we have the location of poles of $\left(\mathcal{M} c_{A, n}^{w}\right)(\lambda, x)$.

From the definition of $f_{n}^{w}(t, x)$, the poles of $\mathcal{M}\left(t^{\ell} \prod_{\alpha}\left(\vartheta^{\alpha_{0}} \partial^{\alpha^{\prime}} w\right)^{A_{\alpha}}\right)(\lambda, x)$ are in $\left\{-\ell-\nu|A|<R e \lambda \leq-\ell-\nu_{0}|A|\right\}$. By $n \leq \ell+\nu_{0}|A|-\nu<n+1$ we have $\ell+\nu_{0}|A|<n+1+\nu$ and $|A|<(n+1+\nu) / \nu_{0}$. Hence $-\ell-\nu|A|>-\frac{\nu}{\nu_{0}}(n+1+\nu)$ and by (4.14) we have the location of poles of $\left(\mathcal{M} f_{n}^{w}\right)(\lambda, x)$.

Set $L^{w}(\cdot)=P(x, \vartheta)+Q^{w}(\cdot)+f^{w}(t, x)$. The coefficients of the operator $L^{w}(\cdot)$ belong to $\mathscr{O}\left(\left(U_{0}-\{0\}\right) \times U^{\prime}\right)$, by replacing another small disk $U_{0}$ and another small polydisk $U^{\prime}$. From now on we treat $L^{w}(v)=0$ and study the behavior of $v(t, x)$ in detail instead of $u(t, x)(=v(t, x)+w(t, x))$ satisfying $L(u)=0$. For the simplicity, by omitting $w$, we denote $L^{w}(\cdot)$ (resp. $Q^{w}(\cdot), c_{A}^{w}(t, x), f^{w}(t, x)$ etc.) by $L(\cdot)\left(\right.$ resp. $Q(\cdot), c_{A}(t, x), f(t, x)$ etc.) The constant $\gamma>0$ is that defined by (1.9) for the original equation.

Let us sum up the conditions of the equation $L(v)=0$ studied in the following sections:

$$
\left\{\begin{array}{l}
L(v)=P(x, \vartheta) v(t, x)+Q(v)+f(t, x)=0 \\
P(x, \vartheta)=\sum_{h=0}^{k} c_{h}(x) \vartheta^{h}, \\
Q(v)=\sum_{A \in \mathbb{N}^{*}} c_{A}(t, x) \prod_{\alpha \in \Delta(m)}\left(\vartheta^{\alpha_{0}} \partial^{\alpha^{\prime}} v(t, x)\right)^{A_{\alpha}},
\end{array}\right.
$$

where

$$
c_{h}(x) \in \mathscr{O}\left(U^{\prime}\right), \quad c_{A}(t, x), f(t, x) \in \mathscr{O}\left(\left(\widetilde{U_{0}-\{0\}}\right) \times U^{\prime}\right)
$$

with

$$
\left\{\begin{aligned}
c_{k}(x) & \neq 0 \\
c_{A}(t, x) & =\sum_{j=0}^{+\infty} c_{A, j}(t, x) \\
f(t, x) & =\sum_{n=0}^{+\infty} f_{n}(t, x)
\end{aligned}\right.
$$


$\left\{c_{A, j}(t, x)\right\}_{(A, j) \in \mathbb{N}^{M^{*}} \times \mathbb{N}},\left\{f_{n}(t, x)\right\}_{n \in \mathbb{N}} \subset \mathcal{M}_{\left\{\Lambda_{-\nu_{0}}(x)\right\}}\left(U^{\prime}\right)$ and for any $\theta^{\prime}>0$

$$
\left\{\begin{array}{c}
\sup _{|\arg t|<\theta^{\prime}, 0<|t|<r_{0},|x|<r^{\prime}}|t|^{-j-e_{A}}\left|c_{A, j}(t, x)\right| \leq C_{0}^{|A|} C_{1}^{j+e_{A}}, \\
\sup _{|\arg t|<\theta^{\prime}, 0<|t|<r_{0},|x|<r^{\prime}}|t|^{-n-\nu}\left|f_{n}(t, x)\right| \leq C_{0} C_{1}^{n} .
\end{array}\right.
$$

As for the distribution of poles of Mellin transform of $c_{A, n}(t, x)$ and $f_{n}(t, x)$ we refer to Proposition 4.3-(2). We assume that $v(t, x) \in \mathscr{O}\left(S \times U^{\prime}\right)$ solves $L(v)=0$ and has a bound

$$
\sup _{(t, x) \in S \times U^{\prime}}|v(t, x)| \leq C|t|^{\nu}
$$

with $\nu>\max \left\{\lambda^{*}, 1\right\}$, where $\lambda^{*}=\max _{1 \leq i \leq k} \sup _{x \in U^{\prime}} \operatorname{Re} \lambda_{i}(x)$.

Finally we give some remarks and inequalities for the later sections.

Remark 4.4. (1) From $\nu \geq \nu_{0}$ and (4.8)

$$
e_{A}+\nu(|A|-1) \geq \gamma\left(m_{A}-k\right) .
$$

We may assume that $\left\{e_{A} ; A \in \mathbb{N}^{M *}\right\}$ is bounded (see also Remark 1.1-(2)).

(2) We note that $e_{A} \geq \min \left\{1, \nu_{0}\right\}>0$ for $|A|=1$ by Lemma 4.1-(1). Hence from $\nu \geq \nu_{0}$ we have

$$
e_{A}+\nu(|A|-1) \geq \min \left\{1, \nu_{0}\right\}>0
$$

for all $A \in \mathbb{N}^{M *}$.

(3) Suppose $\gamma<+\infty$. Take $p \in \mathbb{N}$ such that $\gamma / p \leq \min \left\{1, \nu_{0}\right\}$. Define

$$
\left\{\begin{array}{l}
c_{\gamma}=\gamma / p \\
N_{A, j}=\left[\frac{j+e_{A}+\nu(|A|-1)}{c_{\gamma}}\right] .
\end{array}\right.
$$

We have $0<c_{\gamma} \leq 1$ and

$$
\left\{\begin{array}{l}
\frac{j+e_{A}+\nu(|A|-1)}{c_{\gamma}} \geq j+\frac{\min \left\{1, \nu_{0}\right\}}{c_{\gamma}} \geq j+1, \\
\frac{j+e_{A}+\nu(|A|-1)}{c_{\gamma}} \geq j+p\left(m_{A}-k\right), \\
\frac{j+e_{A}+\nu(|A|-1)}{c_{\gamma}} \geq j+\frac{|A|+1}{2} \text { for }|A| \geq 3 .
\end{array}\right.
$$


Thus we have

$$
\begin{aligned}
& N_{A, j} \geq j+1, \quad N_{A, j} \quad \geq j+p\left(m_{A}-k\right), \\
& N_{A, j} \geq j+|A| / 2,
\end{aligned}
$$

which will be often used.

Suppose $\gamma=+\infty$, that is, $k=m$. Take $c_{\infty}>0$ such that $c_{\infty} \leq \min \left\{1, \nu_{0}\right\}$. Then

$$
\left(j+e_{A}+\nu(|A|-1) / c_{\infty} \geq j+\min \left\{1, \nu_{0}\right\} / c_{\infty} \geq j+1 .\right.
$$

Thus, by defining

$$
N_{A, j}=\left[\frac{j+e_{A}+\nu(|A|-1)}{c_{\infty}}\right],
$$

we have

$$
N_{A, j} \geq j+1, \quad N_{A, j} \geq j+|A| / 2,
$$

which is similar to $(4.22)$.

\section{$\S 5 . \quad$ Asymptotic Terms of Singular Solutions}

In order to show Theorem 1.5 we have to construct asymptotic terms of singular solutions. Let $L(\cdot)$ be the nonlinear operator (4.15) with (4.16), (4.17), (4.18) and (4.20). Let

$$
\begin{aligned}
S & =\left\{t \in \mathbb{C} ; 0<|t|<T^{*},|\arg t|<\theta^{*}\right\}, \\
U^{\prime} & =\left\{x \in \mathbb{C}^{d} ;|x|<R^{\prime}\right\} \quad\left(0<R^{\prime} \leq 1\right) .
\end{aligned}
$$

Let $v(t, x) \in \mathscr{O}\left(S \times U^{\prime}\right)$ be a solution of $L(v)=0$ with (4.19). Now let us try to find functions $\left\{v_{n}(t, x)\right\}_{n \in \mathbb{N}}$ describing the asymptotic behavior of a singular solution $v(t, x)$ near $\{t=0\}$, that is, $v(t, x) \sim \sum_{n=0}^{\infty} v_{n}(t, x)$ as $t \rightarrow$ 0 . The meaning of this expansion is clarified later. Define $\left\{v_{n}(t, x)\right\}_{n \in \mathbb{N}} \subset$ $\mathscr{M}_{\left\{\Lambda_{-\nu_{0}}(x)\right\}}\left(U^{\prime}\right)$ as follows:

$$
\begin{aligned}
& P(x, \vartheta) v_{0}(t, x)+\sum_{0 \leq \ell<c_{\gamma}} f_{\ell}(t, x)=0, \\
& P(x, \vartheta) v_{n}(t, x)+\sum_{c_{\gamma} n \leq \ell<c_{\gamma}(n+1)} f_{\ell}(t, x)+\sum_{\substack{\left.A \in \mathbb{N}^{M *}, n^{\prime}, j \in \mathbb{N}\right\} \\
n^{\prime}+N_{A, j}=n}} c_{A, j}(t, x) \\
& \times\left(\sum_{\left\{\begin{array}{l}
\left(n_{1}, n_{2}, \cdots, n_{|A|}\right) \in \mathbb{N}^{|A|} ; \\
\sum_{i=1}^{|A|} n_{i}=n^{\prime}
\end{array}\right.} \prod_{i=1}^{|A|} \vartheta^{\alpha_{0}(i)} \partial^{\alpha^{\prime}(i)} v_{n_{i}}(t, x)\right)=0 \\
& \text { for } n=1,2, \ldots,
\end{aligned}
$$


where $N_{A, j}=\left[\left(j+e_{A}+\nu(|A|-1)\right) / c_{\gamma}\right]$ (see Remark 4.4). In order to determine $\left\{v_{n}(t, x)\right\}_{n \in \mathbb{N}}$ we need the solvability of $P(x, \vartheta) . \lambda^{*}$ is that defined by (4.1).

Proposition 5.1. $\quad \operatorname{Let} g(t, x) \in \mathscr{O}\left(S \times U^{\prime}\right)$ such that $g(t, x) \ll G|t|^{l} \Theta(R$; $X)$ with $l>\lambda^{*}$, where $X=\sum_{i=1}^{d} x_{i}$. Consider

$$
P(x, \vartheta) v(t, x)=g(t, x) .
$$

Then there exists a unique solution $v(t, x) \in \mathscr{O}\left(S \times U^{\prime}\right)$ such that

$$
v(t, x) \ll C G|t|^{l} \Theta(R ; X) /\left(l-\lambda^{*}\right)^{k} .
$$

Moreover if $g(t, x) \in \mathscr{M}_{\varphi}\left(U^{\prime}\right)\left(\varphi(\lambda, x) \in \mathcal{O}\left(U^{\prime}\right)[\lambda]\right)$, then $v(t, x) \in \mathscr{M}_{\varphi}\left(U^{\prime}\right)$.

Proof. We note that $P(x, \vartheta)$ is an ordinary differential operator with respect to $t$ and $t=0$ is regular singular. First we give a comment about the uniqueness of a solution. Suppose that $v(t, x)$ with $|v(t, x)| \leq C|t|^{l}$ solves $P(x, \vartheta) v(t, x)=0$. Since $t=0$ is regular singular and $l>\lambda^{*}, v(t, x) \equiv 0$.

Now set

$$
\begin{aligned}
G(t, \tau, x) & =\frac{1}{2 \pi i} \int_{\mathcal{C}_{0}} \frac{(t / \tau)^{\lambda} \tau^{-1}}{P(x, \lambda)} d \lambda \\
& =\frac{1}{2 \pi i} \int_{\mathcal{C}_{0}} \frac{(t / \tau)^{\lambda} \tau^{-1}}{c_{k}(x) \prod_{i=1}^{k}\left(\lambda-\lambda_{i}(x)\right)} d \lambda,
\end{aligned}
$$

where $\mathcal{C}_{0}$ is a contour enclosing all the zeroes of $P(x, \lambda)$. Then $P(x, \vartheta) G(t, \tau$, $x)=0,\left.\vartheta^{h} G(t, \tau, x)\right|_{t=\tau}=0$ for $0 \leq h \leq k-2$ and $\left.\vartheta^{k-1} G(t, \tau, x)\right|_{t=\tau}=$ $1 /\left(c_{k}(x) t\right)$. It also holds that for any $\varepsilon>0$ there are $C(\varepsilon)>0$ and $c$ such that for $0<|\tau| \leq|t|$

$$
|G(t, \tau, x)| \leq C(\varepsilon) e^{c|\arg t / \tau|} \frac{|t / \tau|^{\lambda^{*}+\varepsilon}|\log t / \tau|^{k-1}}{|\tau|} .
$$

Set $v(t, x)=\int_{0}^{t} G(t, \tau, x) g(\tau, x) d \tau$. Then $\vartheta^{h} v(t, x)=\int_{0}^{t} \vartheta^{h} G(t, \tau, x) g(\tau, x) d \tau$ for $0 \leq h \leq k-1$ and

$$
\begin{aligned}
\vartheta^{k} v(t, x) & =t \vartheta^{k-1} G(t, t, x) g(t, x)+\int_{0}^{t} \vartheta^{k} G(t, \tau, x) g(\tau) d \tau . \\
& =\frac{g(t, x)}{c_{k}(x)}+\int_{0}^{t} \vartheta^{k} G(t, \tau, x) g(\tau) d \tau .
\end{aligned}
$$

Hence $P(x, \vartheta) v(t, x)=g(t, x)$. If $0<|\tau| \leq|t|$, we have from (5.6)

$$
G(t, \tau, x) \ll C_{0} e^{c|\arg t / \tau|} \frac{|t / \tau|^{\left(l+\lambda^{*}\right) / 2}|\log t / \tau|^{k-1}}{|\tau|} \Theta(R ; X)
$$


and

$$
\begin{aligned}
v(t, x) & \ll C_{1} G|t|^{\left(l+\lambda^{*}\right) / 2}\left(\int_{0}^{|t|}|\tau|^{\left(l-\lambda^{*}\right) / 2-1}(\log |t / \tau|)^{k-1} d|\tau|\right) \Theta(R ; X) \\
& =C_{1} G|t|^{l}\left(\int_{0}^{1}|s|^{\left(l-\lambda^{*}\right) / 2-1}|\log s|^{k-1} d s\right) \Theta(R ; X) \\
& \ll \frac{C G|t|^{l}}{\left(l-\lambda^{*}\right)^{k}} \Theta(R ; X) .
\end{aligned}
$$

Further suppose that $g(t, x) \in \mathscr{M}_{\varphi}\left(U^{\prime}\right)$,

$$
g(t, x)=\frac{1}{2 \pi i} \int_{\mathcal{C}} t^{-\lambda} \frac{\psi(\lambda, x)}{\varphi(\lambda, x)} d \lambda,
$$

where $\varphi(\lambda, x) \in \mathcal{O}\left(U^{\prime}\right)[\lambda]$ such that $\{\lambda ; \varphi(\lambda, x)=0\} \subset\left\{\lambda ; \operatorname{Re} \lambda<-\lambda^{*}\right\}$, $\psi(\lambda, x) \in \mathcal{O}\left(U^{\prime}\right)[\lambda]$ and $\mathcal{C}$ is a contour enclosing all the zeroes of $\varphi(\lambda, x)$. We may assume that the points $\{\lambda ; P(x,-\lambda)=0\}$ are outside of $\mathcal{C}$. Then $v(t, x)$ is represented in another form

$$
v(t, x)=\frac{1}{2 \pi i} \int_{\mathcal{C}} t^{-\lambda} \frac{\psi(\lambda, x)}{P(x,-\lambda) \varphi(\lambda, x)} d \lambda
$$

and there is a $\tilde{\psi}(\lambda, x) \in \mathcal{O}\left(U^{\prime}\right)[\lambda]$ with $\operatorname{deg} \tilde{\psi}(\lambda, x)<\operatorname{deg} \varphi(\lambda, x)$ such that

$$
v(t, x)=\frac{1}{2 \pi i} \int_{\mathcal{C}} t^{-\lambda} \frac{\tilde{\psi}(\lambda, x)}{\varphi(\lambda, x)} d \lambda .
$$

We give how to construct $\tilde{\psi}(\lambda, x)$. We may assume $\operatorname{deg} \varphi(\lambda, x)=p$ and the coefficient of $\lambda^{p}$ is $1 . \tilde{\psi}(\lambda, x)=\sum_{\ell=0}^{p-1} b_{\ell}(x) \lambda^{p-1-\ell}$ is dertermined so that

$$
\frac{1}{2 \pi i} \int_{\mathcal{C}} \frac{\lambda^{s} \psi(\lambda, x)}{P(x,-\lambda) \varphi(\lambda, x)} d \lambda=\frac{1}{2 \pi i} \int_{\mathcal{C}} \frac{\lambda^{s} \tilde{\psi}(\lambda, x)}{\varphi(\lambda, x)} d \lambda
$$

for $0 \leq s \leq p-1$. Since

$$
\begin{aligned}
& \frac{1}{2 \pi i} \int_{\mathcal{C}} \frac{\lambda^{s} \tilde{\psi}(\lambda, x)}{\varphi(\lambda, x)} d \lambda=\frac{1}{2 \pi i} \sum_{\ell=0}^{p-1} b_{\ell}(x) \int_{\mathcal{C}} \frac{\lambda^{p-1-\ell+s}}{\varphi(\lambda, x)} d \lambda \\
= & \frac{1}{2 \pi i} \sum_{\ell=0}^{s-1} b_{\ell}(x) \int_{\mathcal{C}} \frac{\lambda^{p-1-\ell+s}}{\varphi(\lambda, x)} d \lambda+b_{s}(x),
\end{aligned}
$$

$\tilde{\psi}(\lambda, x)$ is determined. Hence $v(t, x) \in \mathscr{M}_{\varphi}\left(U^{\prime}\right)$.

Thus by Proposition $5.1\left\{v_{n}(t, x)\right\}_{n \in \mathbb{N}} \subset \mathscr{M}_{\left\{\Lambda_{-\nu_{0}}(x)\right\}}\left(U^{\prime}\right)$ are successively determined. More precisely we have 
Lemma 5.2. $\quad$ There exist positive constants $a$ and $b$ such that the poles of $\left(\mathcal{M} v_{n}\right)(\lambda, x)$ are contained in $\{\lambda ; \operatorname{Re} \lambda \geq-a n-b\} \cap \Lambda_{-\nu_{0}}(x)$.

Proof. We give the proof by induction on $n$. For $n=0$ the assertion holds for a large $b . v_{n}$ is defined by (5.2). Assume the assertion holds for any $n^{\prime}$ with $n^{\prime}<n$. Then it follows from Lemma 3.1 that the Mellin transform of $\prod_{i=1}^{|A|} \vartheta^{\alpha_{0}(i)} \partial^{\alpha^{\prime}} v_{n_{i}}$ is meromorphic and its poles are contained in $\{\lambda ; \operatorname{Re} \lambda>$ $\left.-a n^{\prime}-b|A|\right\} \cap \Lambda_{-\nu_{0}}(x)$, where $n^{\prime}=\sum_{i=1}^{|A|} n_{i}$. Hence, by Proposition 4.3 the poles of the Mellin transform of $c_{A, j}(t, x) \prod_{i=1}^{|A|} \vartheta^{\alpha_{0}(i)} \partial^{\alpha^{\prime}} v_{n_{i}}$ are contained in $\left\{\lambda ; \operatorname{Re} \lambda>-a n^{\prime}-b|A|-\frac{\nu}{\nu_{0}}\left(j+1+e_{A}\right)\right\} \cap \Lambda_{-\nu_{0}}(x)$. Since $N_{A, j}=n-n^{\prime} \geq 1$ and $\nu>1$, we have $j+e_{A}+|A|-1 \leq n-n^{\prime}+1$, hence $j+e_{A}+|A| \leq n-n^{\prime}+2 \leq$ $3\left(n-n^{\prime}\right)$. Thus we have, by choosing $b \geq \nu / \nu_{0}$ and $a \geq 3 b$,

$$
\begin{aligned}
& a n^{\prime}+b|A|+\left(\nu / \nu_{0}\right)\left(j+1+e_{A}\right) \leq a n^{\prime}+b\left(|A|+j+e_{A}\right)+b \\
\leq & a n^{\prime}+3 b\left(n-n^{\prime}\right)+b \leq a n+b .
\end{aligned}
$$

This implies that the poles of the Mellin transform of $c_{A, j}(t, x) \prod_{i=1}^{|A|} \vartheta^{\alpha_{0}(i)} \partial^{\alpha^{\prime}} v_{n_{i}}$ in (5.2) are contained in $\{\lambda ; \operatorname{Re} \lambda>-a n-b\} \cap \Lambda_{-\nu_{0}}(x)$. The same holds for $\sum_{c_{\gamma} n \leq \ell<c_{\gamma}(n+1)} f_{\ell}(t, x)$ by Proposition 4.3-(2), hence the poles of $\mathcal{M} v_{n}(t, x)$ are in $\{\lambda ; \operatorname{Re} \lambda>-a n-b\} \cap \Lambda_{-\nu_{0}}(x)$ by Proposition 5.1.

Let us return to (5.1). Let $0<T_{0}<T_{1}<T^{*}, 0<\theta_{0}<\theta_{1}<\theta^{*}$ with $\theta_{1}-\theta_{0}<\pi / 6$ and $0<R<R^{\prime}$. $R$ is small, if necessary. As defined in Section 2 , set for $0 \leq \tau \leq 1$

$$
S^{\tau}=\left\{t \in \mathbb{C} ; 0<|t|<(1-\tau) T_{0}+\tau T_{1},|\arg t|<(1-\tau) \theta_{0}+\tau \theta_{1}\right\} .
$$

Then $S^{0} \subset S^{\tau} \subset S^{1} \subset S$. We note $c_{\gamma}=\gamma / p$ for some positive integer $p$ with $p \geq \gamma$, if $\gamma<+\infty$. Let us estimate $\left\{v_{n}(t, x)\right\}_{n \in \mathbb{N}}$.

Proposition 5.3. $\quad$ Suppose $\gamma<\infty$. Then there exist constants $K$ and $L$ such that for $t \in S^{\tau}(0<\tau<1)$

$$
v_{n}(t, x) \ll \frac{K L^{n}|t|^{\frac{\gamma n}{p}+\nu}}{(1-\tau)^{2 m n}} \Theta_{-n k}^{\left(\left[\frac{n}{p}\right]+n k\right)}(R ; X), \quad X=\sum_{i=1}^{d} x_{i},
$$

for $n=0,1,2, \cdots$.

Proof. Let us show (5.8) by induction. It follows from $\left|f_{\ell}(t, x)\right| \leq$ $C_{0} C_{1}^{\ell}|t|^{\ell+\nu}$ (see (4.18)) and Proposition 5.1 that $v_{0}(t, x) \ll K|t|^{\nu} \Theta(R ; X)$ holds for some $K$. Let $n \geq 1$ and assume that (5.8) holds for $n$ replaced by $n^{\prime}$ with 
$0 \leq n^{\prime}<n$. Then it follows from Corollary 2.6 that there exist constants $C^{\prime}$ and $C_{1}$ such that for $n_{i}<n$ and $|\alpha| \leq m$

$$
\begin{aligned}
\vartheta^{\alpha_{0}(i)} \partial^{\alpha^{\prime}(i)} v_{n_{i}}(t, x) & \ll \frac{K C^{\prime} L^{n_{i}}\left(n_{i}+1\right)^{\alpha_{0}(i)}|t|^{\frac{\gamma n_{i}}{p}+\nu}}{(1-\tau)^{2 m n_{i}+\alpha_{0}(i)}} \Theta_{-n_{i} k}^{\left(\left[\frac{n_{i}}{p}\right]+n_{i} k+\left|\alpha^{\prime}(i)\right|\right)}(R ; X) \\
& \ll \frac{K C_{1} L^{n_{i}}|t|^{\frac{\gamma n_{i}}{p}+\nu}}{(1-\tau)^{2 m n_{i}+\alpha_{0}(i)}} \Theta_{-n_{i} k}^{\left(\left[\frac{n_{i}}{p}\right]+n_{i} k+|\alpha(i)|\right)}(R ; X),
\end{aligned}
$$

hence

$$
\prod_{i=1}^{|A|} \vartheta^{\alpha_{0}(i)} \partial^{\alpha^{\prime}(i)} v_{n_{i}}(t, x) \ll \frac{\left(K C_{1}\right)^{|A|} L^{n^{\prime}}|t|^{\frac{\gamma n^{\prime}}{p}+\nu|A|}}{(1-\tau)^{2 m n^{\prime}+m|A|}} \prod_{i=1}^{|A|} \Theta_{-n_{i} k}^{\left(\left[\frac{n_{i}}{p}\right]+n_{i} k+|\alpha(i)|\right)}(R ; X),
$$

where $n^{\prime}=\sum_{i=1}^{|A|} n_{i}$ and $|A|=\sum_{i=1}^{|A|}|\alpha(i)|$. Thus we have

$$
\begin{aligned}
& c_{A, j}(t, x) \prod_{i=1}^{|A|} \vartheta^{\alpha_{0}(i)} \partial^{\alpha^{\prime}(i)} v_{n_{i}}(t, x) \\
\ll & \frac{K^{|A|} L^{n^{\prime}} C_{2}^{|A|+j}|t|^{\frac{\gamma n^{\prime}}{p}}+\nu|A|+e_{A}+j}{(1-\tau)^{2 m n^{\prime}+m|A|}} \prod_{i=1}^{|A|} \Theta_{-n_{i} k}^{\left(\left[\frac{n_{i}}{p}\right]+n_{i} k+m_{A}\right)}(R ; X)
\end{aligned}
$$

for a constant $C_{2}$. It follows from $n=n^{\prime}+N_{A, j} \geq n^{\prime}+j+p\left(m_{A}-k\right)$ and Proposition 2.4-(3) that $n^{\prime} / p \leq n / p+k-m_{A}$ and

$$
\begin{gathered}
\sum_{\left\{\begin{array}{c}
\left(n_{1}, n_{2}, \cdots, n_{|A|}\right) \\
n_{1}+n_{2}+\cdots+n_{|A|}=n^{\prime}
\end{array}\right.} \prod_{i=1}^{|A|} \Theta_{-n_{i} k}^{\left(\left[\frac{n_{i}}{p}\right]+n_{i} k+m_{A}\right)}(R ; X) \ll \frac{C_{3}}{R^{m(|A|-1)}} \Theta_{-n^{\prime} k}^{\left(\left[\frac{n^{\prime}}{p}\right]+n^{\prime} k+m_{A}\right)}(R ; X) \\
\ll \frac{C_{3}}{R^{m(|A|-1)}} \Theta_{-(n-1) k}^{\left(\left[\frac{n^{\prime}}{p}\right]+(n-1) k+m_{A}\right)}(R ; X) \ll \frac{C_{3}}{R^{m(|A|-1)}} \Theta_{-(n-1) k}^{\left(\left[\frac{n}{p}\right]+n k\right)}(R ; X) .
\end{gathered}
$$

By $n^{\prime}+N_{A, j}=n$ and $\frac{\gamma N_{A, j}}{p} \leq j+e_{A}+\nu(|A|-1)$, we have $|t|^{\frac{\gamma n^{\prime}}{p}+\nu|A|+e_{A}+j} \leq$ $|t|^{\frac{\gamma n}{p}+\nu}$ for $|t| \leq 1$ and from Remark 4.4 we have $2 m n^{\prime}+m|A|=2 m\left(n-N_{A, j}+\right.$ 
$|A| / 2) \leq 2 m n$. Thus

$$
\begin{aligned}
I_{n}:= & \sum_{\left\{\begin{array}{c}
\left.A \in \mathbb{N}^{M *}, n^{\prime}, j \in \mathbb{N}\right\} \\
n^{\prime}+N_{A, j}=n
\end{array}\right.} c_{A, j}(t, x) \\
& \times\left(\sum_{\left\{\begin{array}{c}
\left(n_{1}, n_{2}, \cdots, n_{|A|}\right) \in \mathbb{N}^{|A|} ; \\
\sum_{i=1}^{|A|} n_{i}=n^{\prime}
\end{array}\right\}} \prod_{i=1}^{|A|} \vartheta^{\alpha_{0}(i)} \partial^{\alpha^{\prime}(i)} v_{n_{i}}(t, x)\right) \\
\ll & \frac{K L^{n-1} C_{3}}{(1-\tau)^{2 m n}}\left(\sum_{\substack{A \in \mathbb{N}^{M *}, j \in \mathbb{N} \\
1 \leq N_{A, j} \leq n}} \frac{K^{|A|-1} C_{2}^{|A|+j}}{R^{m(|A|-1)} L^{N_{A, j}-1}}\right) \\
& \times|t|^{\frac{\gamma n}{p}+\nu} \Theta_{-(n-1) k}^{\left(\left[\frac{n}{p}\right]+n k\right)}(R ; X) .
\end{aligned}
$$

By choosing $L$ so large, there is a constant $C_{4}$ which is independent of $n$ such that

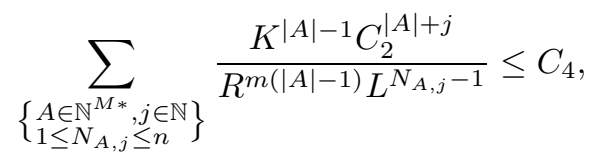

hence

$$
I_{n} \ll \frac{K C_{3} C_{4} L^{n-1}|t|^{\frac{\gamma n}{p}+\nu}}{(1-\tau)^{2 m n}} \Theta_{-(n-1) k}^{\left(\left[\frac{n}{p}\right]+n k\right)}(R ; X) .
$$

By the estimate of $f_{\ell}(t, x)$ (see (4.18)), we have

$$
\begin{aligned}
P(x, \vartheta) v_{n}(t, x) & =-\left(I_{n}+\sum_{c_{\gamma} n \leq \ell<c_{\gamma}(n+1)} f_{\ell}(t, x)\right) \\
& \ll \frac{K C_{5} L^{n-1}|t|^{\frac{\gamma n}{p}+\nu}}{(1-\tau)^{2 m n}} \Theta_{-(n-1) k}^{\left(\left[\frac{n}{p}\right]+n k\right)}(R ; X)
\end{aligned}
$$

and it follows from proposition 5.1 that (5.8) holds for $v_{n}(t, x)$, by taking a large $L$.

By the similar way we have

Proposition 5.4. $\quad$ Suppose $\gamma=\infty(k=m)$. Then there exist constants $K$ and $L$ such that for $t \in S^{\tau}(0<\tau<1)$

$$
v_{n}(t, x) \ll \frac{K L^{n}|t|^{c n+\nu}}{(1-\tau)^{2 m n}} \Theta_{-n m}^{(n m)}(R ; X), \quad X=\sum_{i=1}^{d} x_{i}
$$

for $n=0,1,2, \cdots$, where $c=c_{\infty}$ is that defined in Remark 4.4. 
Set

$$
\mathfrak{r}_{n}(t, x)=v(t, x)-\sum_{\ell=0}^{n} v_{\ell}(t, x)
$$

Our main purpose is to show $\mathfrak{r}_{n}(t, x) \rightarrow 0$ as $t \rightarrow 0$ with estimate, which is done in the following sections. Consequently we'll conclude that $v(t, x) \sim$ $\sum_{\ell=0}^{\infty} v_{\ell}(t, x)$ asymptotically as $t \rightarrow 0$, hence the asymptotic behavior of $v(t, x)$ is characterized by $\left\{v_{\ell}(t, x)\right\}_{\ell \in \mathbb{N}}$.

\section{$\S 6 . \quad$ Remainder Estimate}

In this section we estimate the remainder $\mathfrak{r}_{n}(t, x):=v(t, x)-\sum_{\ell=0}^{n} v_{\ell}(t, x)$ and complete the proof of the main result (Theorem 1.5). Before estimating $\mathfrak{r}_{n}(t, x)$, we first note $S=\left\{t \in \mathbb{C} ; 0<|t|<T^{*},|\arg t|<\theta^{*}\right\}$ is a sector in $t$-space and the sector $S^{\tau}(0 \leq \tau \leq 1)$ is defined (see (5.7)) as in Section 5 . $U^{\prime}=\left\{x \in \mathbb{C}^{d} ;|x|<R^{\prime}\right\}$ is a polydisk in $x$-space and $0<r<R<R^{\prime}<1$ and they are small, if necessaery. From the assumption on $v(t, x)$ we may choose sector $S$ so that

$$
\vartheta^{\alpha_{0}} \partial^{\alpha^{\prime}} v(t, x) \ll K|t|^{\nu} \Theta(R, X) \text { for } t \in S \text { and }|\alpha| \leq m,
$$

where $X=\sum_{i=1}^{d} x_{i}$.

In the following we assume $0<\gamma<+\infty$. If $\gamma=+\infty, \lim _{n \rightarrow+\infty} \mathfrak{v}_{n}(t, x)=$ $\sum_{\ell=0}^{+\infty} v_{\ell}(t, x)$ converges and we can show that $\lim _{n \rightarrow+\infty} \mathfrak{r}_{n}(t, x)=0$ by the similar but less difficult way. It follows from Proposition 5.3 that for $t \in S^{\tau}$

$$
\vartheta^{\alpha_{0}} \partial^{\alpha^{\prime}} v_{n}(t, x) \ll \frac{K L^{n+|\alpha|}|t|^{\frac{\gamma n}{p}+\nu}}{(1-\tau)^{2 m n+|\alpha|}} \Theta_{-n k}^{\left(\left[\frac{n}{p}\right]+n k+|\alpha|\right)}(R ; X)
$$

for $n=0,1,2, \cdots$. We set

$$
\begin{gathered}
q_{A, j}(v)=c_{A, j}(t, x) \prod_{A}\left(\vartheta^{\alpha_{0}} \partial^{\alpha^{\prime}} v\right)^{A_{\alpha}}, \\
\left\{\begin{array}{cl}
\mathcal{N}(n)=\left\{(A, j) \in \mathbb{N}^{M *} \times \mathbb{N} ; N_{A, j} \leq n\right\}, \\
N_{A, j}=\left[\frac{p\left(j+e_{A}+\nu(|A|-1)\right)}{\gamma}\right], & c_{\gamma}=\gamma / p,
\end{array}\right.
\end{gathered}
$$


where $N_{A, j} \geq j+1$ and $N_{A, j} \geq j+p\left(m_{A}-k\right)$ (see Remark 4.4), and

$$
\left\{\begin{aligned}
Q_{n}(v) & =\sum_{(A, j) \in \mathcal{N}(n)} q_{A, j}(v), \\
Q_{n}^{c}(v) & =Q(v)-Q_{n}(v), \\
\mathfrak{v}_{n}(t, x) & =\sum_{\ell=0}^{n} v_{\ell}(t, x), \\
\mathfrak{r}_{n}(t, x) & =v(t, x)-\mathfrak{v}_{n}(t, x) .
\end{aligned}\right.
$$

We have from (6.2) the following estimates of $\mathfrak{v}_{n}(t, x)$ and $\mathfrak{r}_{n}(t, x)$.

Proposition 6.1. $\quad$ Let $0<r<R$ and $0<\tau<1$. Then there is a $\delta_{0}=\delta_{0}(\tau, r)>0$ such that for $t \in S^{\tau}$ with $|t| \leq\left(\delta^{p} /(n+1)\right)^{1 / \gamma}\left(0<\delta \leq \delta_{0}\right)$

$$
\vartheta^{\alpha_{0}} \partial^{\alpha^{\prime}} \mathfrak{v}_{n}(t, x), \vartheta^{\alpha_{0}} \partial^{\alpha^{\prime}} \mathfrak{r}_{n}(t, x) \ll \frac{K_{0} L^{|\alpha|}|t|^{\nu}}{(1-\tau)^{|\alpha|}} \Theta^{(|\alpha|)}(r, X)
$$

hold for $n=0,1,2, \cdots$, where $K_{0}$ depends on $\delta$.

Proof. Suppose $|t| \leq\left(\delta^{p} /(n+1)\right)^{1 / \gamma}$ for a small $\delta>0$. It follows from (6.2) that there is a constant $C_{1}$ such that

$$
\begin{aligned}
\vartheta^{\alpha_{0}} \partial^{\alpha^{\prime}} \mathfrak{v}_{n}(t, x) & \ll \frac{K L^{|\alpha|}|t|^{\nu}}{(1-\tau)^{|\alpha|}} \sum_{\ell=0}^{n} \frac{L^{\ell}|t|^{\gamma \ell / p}}{(1-\tau)^{2 m \ell}} \Theta_{-\ell k}^{\left(\left[\left[\frac{\ell}{p}\right]+\ell k+|\alpha|\right)\right.}(R ; X) \\
& \ll \frac{K L^{|\alpha|}|t|^{\nu}}{(1-\tau)^{|\alpha|}} \sum_{\ell=0}^{n} \frac{L^{\ell}|\delta|^{\ell}}{(1-\tau)^{2 m \ell}(n+1)^{\ell / p}} \frac{\left(\left[\frac{\ell}{p}\right]+\ell k\right) !}{(\ell k) !} \Theta_{-\left[\frac{\ell}{p}\right]-\ell k}^{\left(\left[\frac{\ell}{p}\right]+\ell k+|\alpha|\right)}(R ; X) \\
& \ll \frac{K L^{|\alpha|}|t|^{\nu}}{(1-\tau)^{|\alpha|}} \sum_{\ell=0}^{n} \frac{C_{1}^{\ell}|\delta|^{\ell}}{(1-\tau)^{2 m \ell}} \Theta_{-\left[\frac{\ell}{p}\right]-\ell k}^{\left(\left[\frac{\ell}{p}\right]+\ell k+|\alpha|\right)}(R ; X) .
\end{aligned}
$$

By Proposition 2.4-(4) there is a $\rho_{0}=\rho(r)>0$ such that if $0<\rho \leq \rho_{0}$.

$$
\sum_{\ell=0}^{n} \rho^{\ell} \Theta_{-\left[\frac{\ell}{p}\right]-\ell k}^{\left(\left[\frac{\ell}{p}\right]+\ell k+|\alpha|\right)}(R ; X) \ll K^{\prime} \Theta^{(|\alpha|)}(r ; X) .
$$

Define $\delta_{0}$ by $\rho_{0}=\frac{C_{1} \delta_{0}}{(1-\tau)^{2 m}}$. Then (6.6) holds for $\mathfrak{v}_{n}(t, x)$. We have the estimate about $\mathfrak{r}_{n}(t, x)$ from $(6.1)$ and $\mathfrak{r}_{n}(t, x)=v(t, x)-\mathfrak{v}_{n}(t, x)$, by taking another $L$.

Proposition 6.2. Let $0<r<R$ and $0<\tau<1$. Then there is a $\delta_{1}=\delta_{1}(\tau, r)>0$ such that for $t \in S^{\tau}$ with $|t| \geq\left(\delta^{p} /(n+1)\right)^{1 / \gamma}\left(0<\delta \leq \delta_{1}\right)$,

$$
\begin{aligned}
\vartheta^{\alpha_{0}} \partial^{\alpha^{\prime}} \mathfrak{v}_{n}(t, x), & \vartheta^{\alpha_{0}} \partial^{\alpha^{\prime}} \mathfrak{r}_{n}(t, x) \\
& \ll \frac{K_{0} C_{0}^{n}(n+1)^{(n+1) / p} L^{|\alpha|}|t|^{\gamma(n+1) / p+\nu}}{(1-\tau)^{|\alpha|}} \Theta^{(|\alpha|)}(r ; X)
\end{aligned}
$$


hold for $n=0,1,2, \cdots$, where $K_{0}$ and $C_{0}$ depend on $\delta$.

Proof. Suppose $|t| \geq\left(\delta^{p} /(n+1)\right)^{1 / \gamma}$. By $(6.2)$

$$
\begin{aligned}
& \vartheta^{\alpha_{0}} \partial^{\alpha^{\prime}} \mathfrak{v}_{n}(t, x) \\
& \ll \frac{K L^{|\alpha|}|t|^{\gamma(n+1) / p+\nu}}{(1-\tau)^{|\alpha|}} \sum_{\ell=0}^{n} \frac{L^{\ell}}{(1-\tau)^{2 m \ell}|t|^{\gamma(n+1-\ell) / p}} \Theta_{-\ell k}^{\left(\left[\frac{\ell}{p}\right]+\ell k+|\alpha|\right)}(R ; X) \\
& \ll \frac{K L^{|\alpha|}|t|^{\gamma(n+1) / p+\nu}}{(1-\tau)^{|\alpha|}} \sum_{\ell=0}^{n} \frac{L^{\ell}(n+1)^{(n+1-\ell) / p}}{(1-\tau)^{2 m \ell} \delta^{n+1-\ell}} \Theta_{-\ell k}^{\left(\left[\frac{\ell}{p}\right]+\ell k+|\alpha|\right)}(R ; X) \\
& \ll \frac{K L^{|\alpha|}(n+1)^{(n+1) / p}|t|^{\gamma(n+1) / p+\nu}}{(1-\tau)^{|\alpha|} \delta^{n+1}} \\
& \times \sum_{\ell=0}^{n} \frac{L^{\ell}|\delta|^{\ell}}{(1-\tau)^{2 m \ell}(n+1)^{\ell / p}} \Theta_{-\ell k}^{\left(\left[\frac{\ell}{p}\right]+\ell k+|\alpha|\right)}(R ; X) \\
& \ll \frac{K L^{|\alpha|}(n+1)^{(n+1) / p}|t|^{\gamma(n+1) / p+\nu}}{(1-\tau)^{|\alpha|} \delta^{n+1}} \sum_{\ell=0}^{n} \frac{C_{1}^{\ell}|\delta|^{\ell}}{(1-\tau)^{2 m \ell}} \Theta_{-\left[\frac{\ell}{p}\right]-\ell k}^{\left(\left[\frac{\ell}{p}\right]+\ell k+|\alpha|\right)}(R ; X) .
\end{aligned}
$$

By (6.7) and the same way as the proof of Proposition 6.1, we have (6.8) about $\mathfrak{v}_{n}(t, x)$. From (6.1), $1 \leq|t|^{\gamma(n+1) / p} \frac{(n+1)^{(n+1) / p}}{\delta^{n+1}}$ for $|t| \geq\left(\delta^{p} /(n+1)\right)^{1 / \gamma}$ and $\mathfrak{r}_{n}(t, x)=v(t, x)-\mathfrak{v}_{n}(t, x)$ we have the estimate about $\mathfrak{r}_{n}(t, x)$.

Now let us proceed to obtain another estimate of $\mathfrak{r}_{n}(t, x)$. By relations $v(t, x)=\mathfrak{v}_{n}(t, x)+\mathfrak{r}_{n}(t, x)$, we have

$$
Q_{n}(v)=\sum_{(A, j) \in \mathcal{N}(n)} q_{A, j}\left(\mathfrak{v}_{n-N_{A, j}}+\mathfrak{r}_{n-N_{A, j}}\right)
$$

and $P(x, \vartheta) v(t, x)=P(x, \vartheta)\left(\mathfrak{v}_{n}(t, x)+\mathfrak{r}_{n}(t, x)\right)$, hence

$$
\begin{aligned}
& -P(x, \vartheta)\left(\mathfrak{v}_{n}(t, x)+\mathfrak{r}_{n}(t, x)\right)=Q_{n}(v)+Q_{n}^{c}(v)+f(t, x) \\
= & \sum_{(A, j) \in \mathcal{N}(n)}\left(q_{A, j}\left(\mathfrak{v}_{n-N_{A, j}}+\mathfrak{r}_{n-N_{A, j}}\right)-q_{A, j}\left(\mathfrak{v}_{n-N_{A, j}}\right)\right) \\
& +\sum_{(A, j) \in \mathcal{N}(n)} q_{A, j}\left(\mathfrak{v}_{n-N_{A, j}}\right)+Q_{n}^{c}(v)+f(t, x) .
\end{aligned}
$$

Set

$$
\left\{\begin{array}{c}
I_{n}=\sum_{(A, j) \in \mathcal{N}(n)} q_{A, j}\left(\mathfrak{v}_{n-N_{A, j}}\right)+P(x, \vartheta) \mathfrak{v}_{n}(t, x)+f(t, x), \\
I I_{n}=\sum_{(A, j) \in \mathcal{N}(n)}\left(q_{A, j}\left(\mathfrak{v}_{n-N_{A, j}}+\mathfrak{r}_{n-N_{A, j}}\right)-q_{A, j}\left(\mathfrak{v}_{n-N_{A, j}}\right)\right)
\end{array}\right.
$$


Thus

$$
P(x, \vartheta) \mathfrak{r}_{n}(t, x)+Q_{n}^{c}(v)+I_{n}+I I_{n}=0,
$$

which is an equation $\mathfrak{r}_{n}(t, x)$ satisfies. We obtain an estimate of $\mathfrak{r}_{n}(t, x)$ from (6.11). For this purpose let us estimate $Q_{n}^{c}(v), I_{n}$ and $I I_{n}$.

Proposition 6.3. There exists a $t_{0}>0$ such that for $t \in S$ with $|t| \leq t_{0}$

$$
Q_{n}^{c}(v) \ll K_{0} C_{0}^{n}|t|^{\frac{\gamma(n+1)}{p}+\nu} \Theta(R ; X)
$$

holds for some constants $K_{0}$ and $C_{0}$.

Proof. It follows from (6.1) and (6.3) that there is a constant $C_{1}>0$ such that

$$
q_{A, j}(v) \ll C_{1}^{\nu|A|+j}|t|^{e_{A}+j+\nu|A|} \Theta(R ; X) .
$$

By $N_{A, j} \geq n+1$ for $(A, j) \notin \mathcal{N}(n)$, we have

$$
\sum_{(A, j) \notin \mathcal{N}(n)} q_{A, j}(v) \ll C_{1}^{n+1+\nu}|t|^{\frac{\gamma(n+1)}{p}+\nu}\left(\sum_{(A, j) \notin \mathcal{N}(n)}\left(C_{1} t\right)^{\gamma\left(N_{A, j}-n-1\right) / p}\right) \Theta(R ; X)
$$

and there exist constants $C_{2}$ and $c$ such that $\sum_{\left\{(A, j) ; N_{A, j}=\ell\right\}} 1 \leq C_{2}(1+\ell)^{c}$. Hence there is a $t_{0}$ such that if $|t| \leq t_{0}$,

$$
\begin{aligned}
& \sum_{(A, j) \notin \mathcal{N}(n)}\left(C_{1}|t|\right)^{\gamma\left(N_{A, j}-n-1\right) / p} \leq \sum_{\ell=n+1}^{\infty}\left(\left(C_{1}|t|\right)^{\gamma / p}\right)^{\ell-n-1} \sum_{\left\{(A, j) ; N_{A, j}=\ell\right\}} 1 \\
& \leq C_{2} \sum_{\ell=n+1}^{\infty}(1+\ell)^{c}\left(\left(C_{1}\left|t_{0}\right|\right)^{\gamma / p}\right)^{\ell-n-1} \leq C_{3}(1+n)^{c} .
\end{aligned}
$$

Thus (6.12) holds for some $K_{0}$ and $C_{0}$.

Next let us estimate

$$
I_{n}:=\sum_{(A, j) \in \mathcal{N}(n)} q_{A, j}\left(\mathfrak{v}_{n-N_{A, j}}\right)+P(x, \vartheta) \mathfrak{v}_{n}+f(t, x) .
$$

We have

$$
\begin{gathered}
q_{A, j}\left(\mathfrak{v}_{n-N_{A, j}}\right)=c_{A, j}(t, x) \prod_{i=1}^{|A|}\left(\sum_{n_{i}=0}^{n-N_{A, j}} \vartheta^{\alpha_{0}(i)} \partial^{\alpha^{\prime}(i)} v_{n_{i}}(t, x)\right) \\
=c_{A, j}(t, x)\left(\sum_{\substack{\left(n_{1}, \cdots, n_{|A|}\right) \in \mathbb{N}^{|A|} \\
0 \leq n_{1}, \cdots, n_{|A|} \leq n-N_{A, j}}} \prod_{i=1}^{|A|} \vartheta^{\alpha_{0}(i)} \partial^{\alpha^{\prime}(i)} v_{n_{i}}(t, x)\right)
\end{gathered}
$$


and by the way to determine $\left\{v_{n}(t, x)\right\}_{n \in \mathbb{N}}$ (see (5.2))

$$
\begin{aligned}
& P(x, \vartheta)\left(\sum_{\ell=0}^{n} v_{\ell}(t, x)\right)+\sum_{0 \leq \ell<c_{\gamma}(n+1)} f_{\ell}(t, x) \\
& =-\sum_{(A, j) \in \mathcal{N}(n)} c_{A, j}(t, x) \sum_{n^{\prime}=0}^{n-N_{A, j}}\left(\sum_{\substack{\left(n_{1}, n_{2}, \cdots, n_{|A|}\right) \in \mathbb{N}^{|A|} \\
n_{1}+n_{2}+, \cdots+n_{|A|}=n^{\prime}}} \prod_{i=1}^{|A|} \vartheta^{\alpha_{0}(i)} \partial^{\alpha^{\prime}(i)} v_{n_{i}}(t, x)\right) .
\end{aligned}
$$

Thus we have

$$
\begin{aligned}
& I_{n}=\sum_{(A, j) \in \mathcal{N}(n)} q_{A, j}\left(\mathfrak{v}_{n-N_{A, j}}\right)+\left(f(t, x)-\sum_{0 \leq \ell<c_{\gamma}(n+1)} f_{\ell}(t, x)\right) \\
& -\sum_{(A, j) \in \mathcal{N}(n)} c_{A, j}(t, x)\left(\sum_{n^{\prime}=0}^{n-N_{A, j}}\left(\sum_{\left\{\begin{array}{c}
\left(n_{1}, \cdots, n_{|A|}\right) \in \mathbb{N}^{|A|} \\
\sum_{i=1}^{|A|} n_{i}=n^{\prime}
\end{array}\right.} \prod_{i=1}^{|A|} \vartheta^{\alpha_{0}(i)} \partial^{\alpha^{\prime}(i)} v_{n_{i}}(t, x)\right)\right) .
\end{aligned}
$$

Set

$$
I_{n}\left(A, j, n^{\prime}\right)=c_{A, j}(t, x)\left(\sum_{\left\{\begin{array}{c}
\left(n_{1}, \cdots, n, n_{|A|}\right) \in \mathbb{N}^{|A|} ; \\
0 \leq n_{1}, \cdots, n_{|A|} \leq n-N_{A, j} \\
\sum \sum_{i=1}^{\mid A} n_{i}=n^{\prime}
\end{array}\right.} \prod_{i=1}^{|A|} \vartheta^{\alpha_{0}(i)} \partial^{\alpha^{\prime}(i)} v_{n_{i}}(t, x)\right) .
$$

Then $I_{n}\left(A, j, n^{\prime}\right)=0$ for $n^{\prime}>|A|\left(n-N_{A, j}\right)$ and

$$
\left\{\begin{array}{l}
I_{n}=\left(f(t, x)-\sum_{0 \leq \ell<c_{\gamma}(n+1)} f_{\ell}(t, x)\right)+I_{n}^{0} \\
I_{n}^{0}=\sum_{(A, j) \in \mathcal{N}(n)}\left(\sum_{n^{\prime}>n-N_{A, j}} I_{n}\left(A, j, n^{\prime}\right)\right)
\end{array}\right.
$$

Lemma 6.4. Let $t \in S^{\tau}(0<\tau<1)$ with $|t| \leq\left(\delta^{p} /\left(n-N_{A, j}+1\right)\right)^{1 / \gamma}$. Then there are constants $C_{0}$ and $L_{0}$ such that

$$
\begin{aligned}
I_{n}\left(A, j, n^{\prime}\right) \ll & \frac{C_{0}^{|A|+j} L_{0}^{n^{\prime}} \delta^{n^{\prime}-n+N_{A, j}-1}|t|^{\frac{\gamma(n+1)}{p}}+\nu}{(1-\tau)^{2 m n^{\prime}+m|A|}} \\
& \times\left(n-N_{A, j}+1\right)^{\left(n-N_{A, j}+1\right) / p} \Theta_{-\left[\frac{n^{\prime}}{p}\right]-n^{\prime} k}^{\left(\left[\frac{n^{\prime}}{p}\right]+n^{\prime} k+m_{A}\right)}(R ; X) .
\end{aligned}
$$


Proof. From (6.2) there is a constant $C_{1}$ such that

$$
\begin{aligned}
& H_{A, j}^{n_{1}, \cdots, n_{|A|}}:=c_{A, j}(t, x) \prod_{i=1}^{|A|} \vartheta^{\alpha_{0}(i)} \partial^{\alpha^{\prime}(i)} v_{n_{i}}(t, x) \\
& \ll \frac{K^{|A|} L^{n^{\prime}+m|A|} C_{1}^{|A|+j}|t|^{\frac{\gamma n^{\prime}}{p}+j+e_{A}+\nu|A|}}{(1-\tau)^{2 m n^{\prime}+m|A|}} \prod_{i=1}^{|A|} \Theta_{-n_{i} k}^{\left(\left[\frac{n_{i}}{p}\right]+n_{i} k+m_{A}\right)}(R ; X),
\end{aligned}
$$

where $n^{\prime}=\sum_{i=1}^{|A|} n_{i}$. Let $|t| \leq\left(\delta^{p} /\left(n-N_{A, j}+1\right)\right)^{1 / \gamma}$. Then

$$
\begin{aligned}
& |t|^{\frac{\gamma n^{\prime}}{p}+j+e_{A}+\nu|A|} \leq|t|^{\frac{\gamma(n+1)}{p}+\nu} \times|t|^{\frac{\gamma\left(N_{A, j}+n^{\prime}-n-1\right)}{p}} \\
\leq & |t|^{\frac{\gamma(n+1)}{p}+\nu} \delta^{n^{\prime}-n+N_{A, j}-1}\left(n-N_{A, j}+1\right)^{\left(n-N_{A, j}-n^{\prime}+1\right) / p} .
\end{aligned}
$$

Hence we have

$$
\begin{aligned}
& H_{A, j}^{n_{1}, \cdots, n_{|A|}} \ll \frac{K^{|A|} L^{n^{\prime}+m|A|} C_{1}^{|A|+j} \delta^{n^{\prime}-n+N_{A, j}-1}|t|^{\frac{\gamma(n+1)}{p}+\nu}}{(1-\tau)^{2 m n^{\prime}+m|A|}} \\
& \times\left(n-N_{A, j}+1\right)^{\left(n-N_{A, j}+1\right) / p} \prod_{i=1}^{|A|}\left(n-N_{A, j}+1\right)^{-n_{i} / p} \Theta_{-n_{i} k}^{\left(\left[\frac{n_{i}}{p}\right]+n_{i} k+m_{A}\right)}(R ; X) .
\end{aligned}
$$

Since $n_{i} \leq n-N_{A, j}$,

$$
\begin{aligned}
& \prod_{i=1}^{|A|}\left(n-N_{A, j}+1\right)^{-n_{i} / p} \Theta_{-n_{i} k}^{\left(\left[\frac{n_{i}}{p}\right]+n_{i} k+m_{A}\right)}(R ; X) \\
& \ll \prod_{i=1}^{|A|}\left(n_{i}+1\right)^{-n_{i} / p} \Theta_{-n_{i} k}^{\left(\left[\frac{n_{i}}{p}\right]+n_{i} k+m_{A}\right)}(R ; X) \ll \prod_{i=1}^{|A|} C_{2}^{n_{i}} \Theta_{-\left[\frac{n_{i}}{p}\right]-n_{i} k}^{\left(\left[\frac{n_{i}}{p}\right]+n_{i} k+m_{A}\right)}(R ; X) .
\end{aligned}
$$

Thus there exist constants $C_{3}$ and $L_{1}$ such that

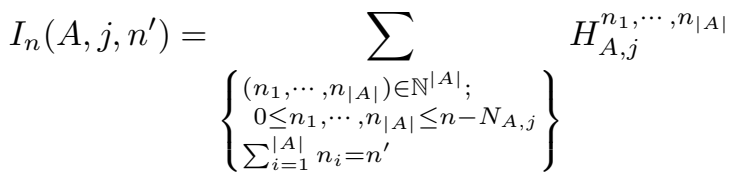

$$
\begin{aligned}
& \ll \frac{C_{3}^{|A|+j} L_{1}^{n^{\prime}} \delta^{n^{\prime}-n+N_{A, j}-1}|t|^{\frac{\gamma(n+1)}{p}+\nu}}{(1-\tau)^{2 m n^{\prime}+m|A|}}\left(n-N_{A, j}+1\right)^{\left(n-N_{A, j}+1\right) / p} \\
& \times\left(\sum_{\left\{\begin{array}{c}
\left(n_{1}, \cdots, n_{|A|}\right) \in \mathbb{N}^{|A|} ; \\
0 \leq n_{1}, \cdots, n_{|A|} \leq n-N_{A, j} \\
\sum_{i=1}^{|A|} n_{i}=n^{\prime}
\end{array}\right.} \prod_{i=1}^{|A|} \Theta_{-\left[\frac{n_{i}}{p}\right]-n_{i} k}^{\left(\left[\frac{n_{i}}{p}+n_{i} k+m_{A}\right)\right.}(R ; X)\right)
\end{aligned}
$$

and (6.16) follows from Proposition 2.4-(3). 
Proposition 6.5. (1) Let $t \in S^{\tau}(0<\tau<1)$. Then there is a $\delta_{2}=$ $\delta_{2}(\tau, r)>0$ such that if $|t| \leq\left(\delta^{p} /(n+1)\right)^{1 / \gamma}$ with $0<\delta \leq \delta_{2}$,

$$
I_{n}^{0} \ll C_{0} C_{1}^{n}|t|^{\frac{\gamma(n+1)}{p}+\nu} \Theta_{-(n-1) k}^{([n / p]+n k)}(r ; X)
$$

holds for some constants $C_{0}$ and $C_{1}$ depending on $\tau$ and $r$.

(2) There are constants $C_{0}$ and $C_{1}$ such that for small $t$

$$
f(t, x)-\sum_{0 \leq \ell<c_{\gamma}(n+1)} f_{\ell}(t, x) \ll C_{0} C_{1}^{n}|t|^{\frac{\gamma(n+1)}{p}+\nu} \Theta(R ; X) .
$$

Proof. (1). Let $0<r<R$. We note $|t| \leq\left(\delta^{p} /(n+1)\right)^{1 / \gamma} \leq\left(\delta^{p} /(n-\right.$ $\left.\left.N_{A, j}+1\right)\right)^{1 / \gamma}$. We have from lemma 6.4 , by setting $L_{0}(\tau)=L_{0} /(1-\tau)^{2 m}$ and $C_{0}(\tau)=C_{0} /(1-\tau)^{m}$,

$$
\begin{aligned}
& I_{n}^{0}= \sum_{(A, j) \in \mathcal{N}(n)}\left(\sum_{n^{\prime}>n-N_{A, j}} I_{n}\left(A, j, n^{\prime}\right)\right) \\
& \ll|t|^{\frac{\gamma(n+1)}{p}+\nu} \sum_{(A, j) \in \mathcal{N}(n)} C_{0}^{|A|+j}(\tau)\left(n-N_{A, j}+1\right)^{\left(n-N_{A, j}+1\right) / p} \\
& \times\left(\sum_{n^{\prime}>n-N_{A, j}} L_{0}(\tau)^{n^{\prime}} \delta^{n^{\prime}-n+N_{A, j}-1} \Theta_{-\left[\frac{n^{\prime}}{p}\right]-n^{\prime} k}^{\left(\left[\frac{n^{\prime}}{p}\right)+n^{\prime} k+m_{A}\right)}(R ; X)\right) .
\end{aligned}
$$

It follows from Propsition 2.4 that for small $\delta=\delta(\tau, r)>0$ there exists a constant $C_{1}$ such that,

$$
\begin{aligned}
& \left(\sum_{n^{\prime}>n-N_{A, j}} L_{0}(\tau)^{n^{\prime}} \delta^{n^{\prime}-n+N_{A, j}-1} \Theta_{-\left[\frac{n^{\prime}}{p}\right]-n^{\prime} k}^{\left(\left[\frac{n^{\prime}}{p}+n^{\prime} k+m_{A}\right)\right.}(R ; X)\right) \\
& \ll L_{0}(\tau)^{n-N_{A, j}} \delta^{-1}\left(\sum_{n^{\prime} \geq n-N_{A, j}}\left(L_{0}(\tau) \delta\right)^{n^{\prime}-n+N_{A, j}} \Theta_{-\left[\frac{n^{\prime}}{p}\right]-n^{\prime} k}^{\left(\left[\frac{n^{\prime}}{p}\right)+n^{\prime} k+m_{A}\right)}(R ; X)\right) \\
& \ll C_{1} L_{0}(\tau)^{n-N_{A, j}} \Theta_{\left.-\left[\left(n-N_{A, j}\right) / p\right]+\left(n-N_{A, j}\right) k+p\right]-\left(n-N_{A, j}\right) k}^{([(n)}(r ; X) .
\end{aligned}
$$

Hence, by Proposition 2.3 and choosing $L_{1}>L_{0}(\tau)$, we have

$$
\begin{aligned}
I_{n}^{0} \ll & C_{1}|t|^{\frac{\gamma(n+1)}{p}+\nu} \sum_{(A, j) \in \mathcal{N}(n)} C_{0}^{|A|+j}(\tau) L_{0}(\tau)^{n-N_{A, j}} \\
& \times\left(n-N_{A, j}+1\right)^{\left(n-N_{A, j}+1\right) / p} \Theta_{-\left[\left(n-N_{A, j}\right) / p\right]-\left(n-N_{A, j}\right) k}^{\left(\left[\left(n-N_{A}\right) / p\right]+\left(n-N_{A}\right) k+m_{A}\right)}(r ; X) \\
\ll & C_{1}|t|^{\frac{\gamma(n+1)}{p}+\nu} \sum_{(A, j) \in \mathcal{N}(n)} C_{0}^{|A|+j}(\tau) L_{1}^{n-N_{A, j}} \Theta_{-\left(n-N_{A, j}\right) k}^{\left(\left[\left(n-N_{A, j}\right) /\left(n-N_{A, j}\right) k+m_{A}\right)\right.}(r ; X) \\
\ll & \ll C_{1}|t|^{\frac{\gamma(n+1)}{p}+\nu} L_{1}^{n-1}\left(\sum_{(A, j) \in \mathcal{N}(n)} C_{0}^{|A|+j}(\tau) L_{1}^{1-N_{A, j}}\right) \Theta_{-(n-1) k}^{([n / p]+n k)}(r ; X) .
\end{aligned}
$$


If $C_{0} / L_{1}^{2}<1 / 2$, then by $|A|+j \leq 2 N_{A, j}$ there exists a constant $C_{2}$ which is independent of $n$ such that

$$
\sum_{(A, j) \in \mathcal{N}(n)} L_{1}^{1-N_{A, j}} C_{0}^{|A|+j} \leq \sum_{\ell=1}^{n} C_{0}^{2 \ell} L_{1}^{1-\ell} \sum_{\left\{(A, j) ; N_{A, j}=\ell\right\}} 1 \leq C_{2},
$$

hence (6.17) holds.

(2) As for $f(t, x)-\sum_{0 \leq \ell<c_{\gamma}(n+1)} f_{\ell}(t, x)$, we have from (4.18)

$$
\begin{aligned}
& f(t, x)-\sum_{\ell<c_{\gamma}(n+1)} f_{\ell}(t, x)=\sum_{\ell \geq c_{\gamma}(n+1)} f_{\ell}(t, x) \ll C^{\prime}|t|^{\nu} \sum_{\ell \geq c_{\gamma}(n+1)}^{\infty} C^{\ell}|t|^{\ell} \Theta(R ; X) \\
& \ll C_{0}|t|^{\frac{\gamma(n+1)}{p}+\nu} C_{1}^{n} \Theta(R ; X) .
\end{aligned}
$$

Since $I_{n}=I_{n}^{0}+\left(f(t, x)-\sum_{0 \leq \ell<c_{\gamma}(n+1)} f_{\ell}(t, x)\right)$, we have

Corollary 6.6. Let $t \in S^{\tau}(0<\tau<1)$. Then there is a $\delta_{2}=\delta_{2}(\tau, r)>$ 0 such that if $|t| \leq\left(\delta^{p} /(n+1)\right)^{1 / \gamma}$ with $0<\delta \leq \delta_{2}$,

$$
I_{n} \ll C_{0} C_{1}^{n}|t|^{\frac{\gamma(n+1)}{p}+\nu} \Theta_{-(n-1) k}^{[n / p]+n k}(r ; X)
$$

holds.

Now let us proceed to give another estimate of $\mathfrak{r}_{n}(t, x)$. Fix $\tau=\tau_{0}(0<$ $\left.\tau_{0}<1\right)$ and let $\delta_{*}=\min \left\{\delta_{0}\left(r, \tau_{0}\right), \delta_{1}\left(r, \tau_{0}\right), \delta_{2}\left(r, \tau_{0}\right)\right\}$, where $\delta_{0}\left(r, \tau_{0}\right), \delta_{1}\left(r, \tau_{0}\right)$ and $\delta_{2}\left(r, \tau_{0}\right)$ are those defined in Propostions 6.1 and 6.2 and Corollary 6.6 respectively. Let $T=\{t ; 0<|t|<\tilde{T},|\arg t|<\tilde{\theta}\}$ be a sector such that $T \Subset S^{\tau_{0}}$. By taking $0<\tilde{\theta}_{0}<\tilde{\theta_{1}}<\tilde{\theta}$ and $0<\tilde{T}_{0}<\tilde{T}_{1}<\tilde{T}$ we can define $T^{\tau}$ such as $S^{\tau}$. We have

Proposition 6.7. Let $t \in T^{\tau}(0<\tau<1)$. Then if $|t| \leq\left(\delta^{p} /(n+1)\right)^{1 / \gamma}$ with $0<\delta \leq \delta_{*}$,

$$
\mathfrak{r}_{n}(t, x) \ll \frac{K_{*} C_{*}^{n+1}|t|^{\frac{\gamma(n+1)}{p}+\nu}}{(1-\tau)^{m n}} \Theta_{-n k}^{\left(\left[\frac{n}{p}\right]+n k\right)}(r ; X)
$$

holds, where $K_{*}$ and $C_{*}$ depends on $\delta$ and $\tau$.

Proposition 6.7 follows from Lemmas $6.8,6.9$ and 6.10 given in the following, where induction on $n$ is used. Before its proof we complete the proof of 
Theorem 1.5, by obtaining an estimate of $\mathfrak{r}_{n}(t, x)$ of Gevrey type, which follows from Propositions 6.2 and 6.7 .

Proof of Theorem 1.5. Fix $\delta$ with $0<\delta \leq \delta_{*}$ and $\tau=\tau_{1}\left(0<\tau_{1}<1\right)$. Let $t \in T^{\tau_{1}}$. It follows from Propositions 6.2 and 6.7 that there are constants $K_{0}$ and $C_{0}$ such that for $t \in T^{\tau_{1}}$

$$
\left\{\begin{array}{l}
\mathfrak{r}_{n}(t, x) \ll K_{0} C_{0}^{n}(n+1)^{(n+1) / p}|t|^{\frac{\gamma(n+1)}{p}+\nu} \Theta(r ; X) \quad \text { for }|t| \geq\left(\delta^{p} /(n+1)\right)^{1 / \gamma} \\
\mathfrak{r}_{n}(t, x) \ll K_{0} C_{0}^{n}|t|^{\frac{\gamma(n+1)}{p}+\nu} \Theta_{-n k}^{\left(\left[\frac{n}{p}\right]+n k\right)}(r ; X) \quad \text { for }|t| \leq\left(\delta^{p} /(n+1)\right)^{1 / \gamma}
\end{array}\right.
$$

Hence if $\sum_{i=1}^{d}\left|x_{i}\right|<r / 2$, by Propositon 2.3-(5) there are constants $C_{1}$ and $C^{\prime}$ such that

$$
\left|\mathfrak{r}_{n}(t, x)\right| \leq C^{\prime} C_{1}^{n}|t|^{\frac{\gamma(n+1)}{p}+\nu} \Gamma\left(\frac{n+1}{p}+1\right) .
$$

Recall $u(t, x)=v(t, x)+w(t, x)$ and let $u_{0}(t, x)=v_{0}(t, x)+w(t, x)$ and $u_{n}(t, x)=v_{n}(t, x)$ for $n \geq 1$. Then

$$
u(t, x)-\sum_{n=0}^{N-1} u_{n}(t, x)=v(t, x)-\sum_{n=0}^{N-1} v_{n}(t, x)=\mathfrak{r}_{N-1}(t, x)
$$

and there are other constants $C_{0}$ and $C$ such that

$$
\left|\mathfrak{r}_{N-1}(t, x)\right| \leq C_{0} C^{N}|t|^{\frac{\gamma N}{p}+\nu} \Gamma\left(\frac{N}{p}+1\right) \leq C_{0} C^{N}|t|^{p_{N}+\nu_{0}} \Gamma\left(\frac{p_{N}}{\gamma}+1\right),
$$

where $p_{N}=\gamma N / p$. The locations of poles of $\left(\mathcal{M} u_{n}\right)(\lambda, x)$ follows from Lemma 5.2 .

We have estimates of $Q_{n}^{c}$ and $I_{n}$. In odrer to show Proposition 6.7 we estimate $I I_{n}$ (see (6.11)) by induction on $n$. First let us consider $q_{A, j}\left(\mathfrak{v}_{n-N_{A, j}}+\right.$ $\left.\mathfrak{r}_{n-N_{A, j}}\right)-q_{A, j}\left(\mathfrak{v}_{n-N_{A, j}}\right)$. We have

$$
\begin{aligned}
& q_{A, j}\left(\mathfrak{v}_{n-N_{A, j}}+\mathfrak{r}_{n-N_{A, j}}\right)-q_{A, j}\left(\mathfrak{v}_{n-N_{A, j}}\right) \\
= & c_{A, j}(t, x) \sum_{\substack{s=\left(s_{\alpha}\right) \in \mathbb{N}^{M *} \\
s \leq A}}\left(\begin{array}{c}
A \\
s
\end{array}\right) \prod_{\alpha}\left(\vartheta^{\alpha_{0}} \partial^{\alpha^{\prime}} \mathfrak{v}_{n-N_{A, j}}\right)^{A_{\alpha}-s_{\alpha}}\left(\vartheta^{\alpha_{0}} \partial^{\alpha^{\prime}} \mathfrak{r}_{n-N_{A, j}}\right)^{s_{\alpha}} .
\end{aligned}
$$

For $s=\left(s_{\alpha}\right) \in \mathbb{N}^{M *}$, there is an $\hat{\alpha}=\left(\hat{\alpha}_{0}, \hat{\alpha}^{\prime}\right) \in \mathbb{N}^{d+1}$ such that $s_{\hat{\alpha}} \geq 1$. Hence

$$
q_{A, j}\left(\mathfrak{v}_{n-N_{A, j}}+\mathfrak{r}_{n-N_{A, j}}\right)-q_{A, j}\left(\mathfrak{v}_{n-N_{A, j}}\right)=\sum_{\substack{s=\left(s_{\alpha}\right) \in \mathbb{N}^{M *} \\ s \leq A}} \hat{q}_{A, j}(n ; s),
$$


where

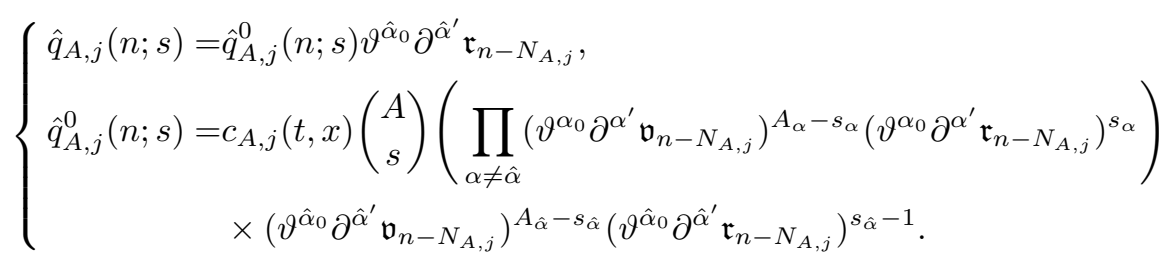

Lemma 6.8. Let $t \in S^{\tau_{0}}$ and $0<\delta \leq \delta_{*}$. Then there exist constants $K$ and $C$ such that for $t$ with $|t| \leq\left(\delta^{p} /(n+1)\right)^{1 / \gamma}$

$$
\hat{q}_{A, j}^{0}(n ; s) \ll K C^{|A|+j}|t|^{\frac{\gamma N_{A, j}}{p}} \Theta^{\left(m_{A}\right)}(r ; X) .
$$

Proof. From Proposition 6.1, for $|\alpha| \leq m_{A}$

$$
\vartheta^{\alpha_{0}} \partial^{\alpha^{\prime}} \mathfrak{v}_{n}(t, x), \vartheta^{\alpha_{0}} \partial^{\alpha^{\prime}} \mathfrak{r}_{n}(t, x) \ll \frac{K_{0} L^{m_{A}}|t|^{\nu}}{\left(1-\tau_{0}\right)^{m_{A}}} \Theta^{\left(m_{A}\right)}(r ; X) .
$$

Hence there exist constants $C_{1}, K$ and $C$ such that

$$
\begin{aligned}
\hat{q}_{A, j}^{0}(n ; s) & \ll C_{1}{ }^{|A|+j}|t|^{e_{A}+j} \Theta(R ; X)\left(\frac{K_{0} L^{m_{A}}|t|^{\nu}}{\left(1-\tau_{0}\right)^{m_{A}}} \Theta^{\left(m_{A}\right)}(r ; X)\right)^{|A|-1} \\
& \ll K C^{|A|+j}|t|^{\frac{\gamma N_{A, j}}{p}} \Theta^{\left(m_{A}\right)}(r ; X) .
\end{aligned}
$$

Lemma 6.9. Suppose that (6.20) holds for $n$ replaced by $n-N_{A, j}$. Then for $t \in T^{\tau}(0<\tau<1)$ with $|t| \leq\left(\delta^{p} /(n+1)\right)^{1 / \gamma}\left(0<\delta \leq \delta_{*}\right)$ there exists a constant $C_{0}$ such that

$$
\begin{aligned}
& \sum_{\substack{s=\left(s_{\alpha}\right) \in \mathbb{N}^{M *} \\
s \leq A}} \hat{q}_{A, j}(n ; s) \\
\ll & \frac{K_{*} C_{0}^{|A|+j} C_{*}^{n+1-N_{A, j}}|t|^{\frac{\gamma(n+1)}{p}+\nu}}{(1-\tau)^{m n}} \Theta_{-(n-1) k}^{([n / p]+n k)}(r ; X) .
\end{aligned}
$$

Proof. It follows from the assumption (see (6.20)) that there is a constant $C_{1}$ such that

$$
\begin{aligned}
& \partial^{\hat{\alpha}^{\prime}} \mathfrak{r}_{n-N_{A, j}} \\
\ll & \frac{K_{*} C_{1} C_{*}^{n+1-N_{A, j}}|t|^{\frac{\gamma\left(n+1-N_{A, j}\right)}{p}+\nu}}{(1-\tau)^{m\left(n-N_{A, j}\right)}} \Theta_{-\left(n-N_{A, j}\right) k}^{\left(\left[\left(n-N_{A, j}\right) / p\right]+\left(n-N_{A, j}\right) k+\left|\hat{\alpha}^{\prime}\right|\right)}(r ; X) .
\end{aligned}
$$


Put $t_{0}=\left(\delta^{p} /(n+1)\right)^{1 / \gamma}$ and $t_{1}=\left(\delta^{p} /\left(n-N_{A, j}+1\right)\right)^{1 / \gamma}$ in Proposition 2.5. Then it follows from

$$
\frac{1}{(1+x)^{1 / \gamma}-1} \leq C^{\prime \prime} \max \left\{x^{-1}, 1\right\} \text { for } x>0
$$

that there exists a constant $C^{\prime}$ such that

$$
\frac{t_{0}}{t_{1}-t_{0}}=\frac{1}{\left(\frac{n+1}{n-N_{A, j}+1}\right)^{1 / \gamma}-1}=\frac{1}{\left(1+\frac{N_{A, j}}{n-N_{A, j}+1}\right)^{1 / \gamma}-1} \leq C^{\prime}\left(n-N_{A, j}+1\right) .
$$

Therefore, it follows from Proposition 2.5 that there are constants $C_{2}$ and $C_{3}$ such that for $t \in T^{\tau}$ with $|t| \leq\left(\delta^{p} /(n+1)\right)^{1 / \gamma}$

$$
\begin{aligned}
& \vartheta^{\hat{\alpha}_{0}} \partial^{\hat{\alpha}^{\prime}} \mathfrak{r}_{n-N_{A, j}} \\
\ll & \frac{K_{*} C_{2} C_{*}^{n+1-N_{A, j}}|t|^{\frac{\gamma\left(n+1-N_{A, j}\right)}{p}+\nu}}{(1-\tau)^{m\left(n-N_{A, j}\right)+\left|\hat{\alpha}_{0}\right|}}\left(n-N_{A, j}+1\right)^{\hat{\alpha}_{0}} \\
& \times \Theta_{-\left(n-N_{A, j}\right) k}^{\left(\left[\left(n-N_{A, j}\right) / p\right]+\left(n-N_{A, j}\right) k+|+| \hat{\alpha}^{\prime} \mid\right)}(r ; X) \\
\ll & \frac{K_{*} C_{3} C_{*}^{n+1-N_{A, j}}|t|^{\frac{\gamma\left(n+1-N_{A, j}\right)}{p}+\nu}}{(1-\tau)^{m\left(n-N_{A, j}\right)+|\hat{\alpha}|}} \Theta_{-\left(n-N_{A, j}\right) k}^{\left(\left[\left(n-N_{A, j}\right) / p\right]+\left(n-N_{A, j}\right) k+|\hat{\alpha}|\right)}(r ; X) .
\end{aligned}
$$

Since $N_{A, j} \geq j+p\left(m_{A}-k\right)$ and $|\hat{\alpha}| \leq m_{A}$, we have $\left[\left(n-N_{A, j}\right) / p\right] \leq[(n-$ $j) / p]+k-m_{A}$ and

$$
\begin{aligned}
& {\left[\left(n-N_{A, j}\right) / p\right]+\left(n-N_{A, j}\right) k+|\hat{\alpha}| } \\
\leq & {[(n-j) / p]+k-m_{A}+\left(n-N_{A, j}\right) k+|\hat{\alpha}| } \\
\leq & {[(n-j) / p]+\left(n-N_{A, j}+1\right) k, }
\end{aligned}
$$

hence

$$
\begin{aligned}
& \Theta_{-\left(n-N_{A, j}\right) k}^{\left[\left(\left[n-N_{A, j}\right) / p\right]+\left(n-N_{A, j}\right) k+|\hat{\alpha}|\right.}(r ; X) \\
& \ll \Theta_{-\left(n-N_{A, j}\right) k}^{\left([n / p]+\left(n-N_{A, j}+1\right) k\right)}(r ; X) \ll \Theta_{-(n-1) k}^{([n / p]+n k)}(r ; X) .
\end{aligned}
$$

It follows from Lemma 6.8 and $m\left(n-N_{A, j}\right)+m_{A} \leq m n$ that there is a constant $C_{0}$ such that

$$
\begin{aligned}
& \sum_{\substack{s=\left(s_{\alpha}\right) \in \mathbb{N}^{M *} \\
s \leq A}} \hat{q}_{A, j}(n, s)=\sum_{\substack{s=\left(s_{\alpha}\right) \in \mathbb{N}^{M *} \\
s \leq A}} \hat{q}_{A, j}^{0}(n, s) \vartheta^{\hat{\alpha}_{0}} \partial^{\hat{\alpha}^{\prime}} \mathfrak{r}_{n-N_{A, j}} \\
& \ll \frac{K_{*} C_{0}^{|A|+j} C_{*}^{n+1-N_{A, j}}|t|^{\frac{\gamma(n+1)}{p}+\nu}}{(1-\tau)^{m n}} \Theta_{-(n-1) k}^{([n / p]+n k)}(r ; X) .
\end{aligned}
$$


Lemma 6.10. Suppose that (6.20) holds for $n$ replaced by $n^{\prime}$ with $0 \leq$ $n^{\prime} \leq n-1$. If the constant $C_{*}$ in (6.20) is large, then there exists a constant $C^{\prime}$ such that for $t \in T^{\tau}(0<\tau<1)$ with $|t| \leq\left(\delta^{p} /(n+1)\right)^{1 / \gamma}\left(0<\delta \leq \delta_{*}\right)$

$$
I I_{n} \ll \frac{K_{*} C^{\prime} C_{*}^{n}|t|^{\frac{\gamma(n+1)}{p}+\nu}}{(1-\tau)^{m n}} \Theta_{-(n-1) k}^{\left(\left[\frac{n}{p}\right]+n k\right)}(r ; X) .
$$

Proof. From Lemma 6.9 there exists a constant $C_{1}$ such that

$$
\begin{aligned}
& I I_{n}=\sum_{(A, j) \in \mathcal{N}(n)}\left(q_{A, j}\left(\mathfrak{v}_{n-N_{A, j}}+\mathfrak{r}_{n-N_{A, j}}\right)-q_{A, j}\left(\mathfrak{v}_{n-N_{A, j}}\right)\right) \\
& =\sum_{(A, j) \in \mathcal{N}(n)} \sum_{\substack{\left.s=\left(s_{\alpha}\right) \in \mathbb{N}^{M *}\right\} \\
s \leq A}} \hat{q}_{A, j}(n ; s) \\
& \ll \frac{K_{*}|t|^{\frac{\gamma(n+1)}{p}+\nu}}{(1-\tau)^{m n}}\left(\sum_{(A, j) \in \mathcal{N}(n)} C_{0}^{|A|+j} C_{*}^{n+1-N_{A, j}}\right) \Theta_{-(n-1) k}^{\left(\left[\frac{n}{p}\right]+n k\right)}(r ; X) \\
& \ll \frac{K_{*} C_{*}^{n}|t|^{\frac{\gamma(n+1)}{p}+\nu}}{(1-\tau)^{m n}}\left(\sum_{(A, j) \in \mathcal{N}(n)} C_{0}^{j+|A|} C_{*}^{1-N_{A, j}}\right) \Theta_{-(n-1) k}^{\left(\left[\frac{n}{p}\right]+n k\right)}(r ; X) \text {. }
\end{aligned}
$$

By taking $C_{*}$ so large, there exists a constant $C^{\prime}$ such that

$$
\sum_{(A, j) \in \mathcal{N}(n)} C_{0}^{|A|+j} C_{*}^{1-N_{A, j}} \leq \sum_{\ell=1}^{n} C_{0}^{2 \ell} C_{*}^{1-\ell} \sum_{\left\{(A, j) ; N_{A, j}=\ell\right\}} 1 \leq C^{\prime},
$$

from which (6.23) follows.

Proof of Proposition 6.7. Let $0<\delta \leq \delta_{*}$. Then it follows from Proposition 6.3, Corollary 6.6 and Lemma 6.10 that there are constants $K_{*}, C_{*}$ and $C^{\prime}$ such that

$$
Q_{n}^{c}(v)+I_{n}+I I_{n} \ll \frac{K_{*} C^{\prime} C_{*}^{n}|t|^{\frac{\gamma(n+1)}{p}+\nu}}{(1-\tau)^{m n}} \Theta_{(n-1) k}^{\left(\left[\frac{n}{p}\right]+n k\right)}(r ; X)
$$

for $t \in T^{\tau}$ with $|t| \leq\left(\delta^{p} /(n+1)\right)^{1 / \gamma}$, hence it follows from Proposition 5.1 that

$$
\mathfrak{r}_{n}(t, x) \ll \frac{K_{*} C^{\prime \prime} C_{*}^{n}|t|^{\frac{\gamma(n+1)}{p}+\nu}}{(1-\tau)^{m n}} \Theta_{-n k}^{\left(\left[\frac{n}{p}\right]+n k\right)}(r ; X) .
$$

We take $C^{*}$ with $C^{*} \geq C^{\prime \prime}$ and have (6.20). 


\section{§7. $\quad$ Majorant Functions 2}

In this section we give the proofs of Lemmas and Propositions in Section 2 concering majorant functions.

Proof of Lemma 2.1. (1) Let $0<r^{\prime}<1$ and $C\left(r^{\prime}\right)=\sup _{n \geq 0}(1+n)^{m+2} r^{\prime n}$. Then $(1+n)^{-m-2} \leq 1 \leq C\left(r^{\prime}\right)(1+n)^{-m-2} r^{\prime-n}$, from which (2.2) follows.

(2) $\operatorname{By} \theta^{(p+1)}(X)=c \sum_{n=0}^{\infty} \frac{(n+p+1)(n+p) \cdots(n+1)}{(n+p+2)^{m+2}} X^{n}$ and $\frac{(n+p+1)^{m+3}}{(n+p+2)^{m+2}} \geq(p+$ 1) $\left(\frac{n+p+1}{n+p+2}\right)^{m+2} \geq \frac{p+1}{2^{m+2}}$, we have $(p+1) \theta^{(p)}(X) \ll 2^{m+2} \theta^{(p+1)}(X)$.

(3) Let $0 \leq \ell_{1} \leq \ell_{2} \leq m$. Then

$$
\begin{aligned}
& \theta^{\left(\ell_{1}\right)}(X) \theta^{\left(\ell_{2}\right)}(X) \\
\ll & c^{2}\left(\sum_{n_{1}=0}^{+\infty} \frac{X^{n_{1}}}{\left(n_{1}+\ell_{1}+1\right)^{m-\ell_{1}+2}}\right)\left(\sum_{n_{2}=0}^{+\infty} \frac{X^{n_{2}}}{\left(n_{2}+\ell_{2}+1\right)^{m-\ell_{2}+2}}\right) \\
\ll & c^{2} \sum_{n=0}^{+\infty}\left(\sum_{n_{1}+n_{2}=n} \frac{X^{n}}{\left(n_{1}+\ell_{1}+1\right)^{m-\ell_{1}+2}\left(n_{2}+\ell_{2}+1\right)^{m-\ell_{2}+2}}\right) .
\end{aligned}
$$

The inequality

$$
\begin{aligned}
& \left(\sum_{n_{1}+n_{2}=n} \frac{1}{\left(n_{1}+\ell_{1}+1\right)^{m-\ell_{1}+2}\left(n_{2}+\ell_{2}+1\right)^{m-\ell_{2}+2}}\right) \\
& \leq \frac{C^{\prime}}{\left(n+\ell_{2}+1\right)^{m-\ell_{2}+2}} \leq C \frac{\left(n+\ell_{2}\right)\left(n+\ell_{2}-1\right) \cdots(n+1)}{\left(n+\ell_{2}+1\right)^{m+2}}
\end{aligned}
$$

means that $\theta^{\left(\ell_{1}\right)}(X) \theta^{\left(\ell_{2}\right)}(X) \ll c C \theta^{\left(\ell_{2}\right)}(X)$. Hence choose $c>0$ such that $c C \leq 1$.

In the following we fix $c>0$ in $\theta(X)$ so that $\theta^{\left(\ell_{1}\right)}(X) \theta^{\left(\ell_{2}\right)}(X) \ll \theta^{\left(\ell_{2}\right)}(X)$ holds for $0 \leq \ell_{1} \leq \ell_{2} \leq m$ (see (2.4)).

Proof of Lemma 2.2. By $(2.4) \prod_{i=1}^{r} \theta^{\left(\ell_{i}\right)}(X) \ll \theta^{\left(\ell^{\prime}\right)}(X)$ holds. Differetiating it $p$-times, we have $\prod_{i=1}^{r} \theta^{\left(p_{i}+\ell_{i}\right)}(X) \ll \theta^{\left(p+\ell^{\prime}\right)}(X)$. By differentiating it again $q$-times

$$
\sum_{\left\{\begin{array}{c}
\left(q_{1}, q_{2}, \cdots, q_{r}\right) \in \mathbb{N}^{r} \\
q_{1}+q_{2}+\cdots+q_{r}=q
\end{array}\right\}} \frac{q !}{q_{1} ! q_{2} ! \cdots q_{r} !} \prod_{i=1}^{r} \theta^{\left(p_{i}+q_{i}+\ell_{i}\right)}(X) \ll \theta^{\left(p+q+\ell^{\prime}\right)}(X),
$$

which implies (2.5).

We have defined for $0<R<1$ (see $(2.6)$ )

$$
\Theta_{-q}^{(p)}(R ; X)=\frac{1}{q !}\left(\frac{d}{d X}\right)^{p} \theta\left(\frac{X}{R}\right)=\frac{1}{R^{p} q !} \theta^{(p)}\left(\frac{X}{R}\right) .
$$


Proof of Proposition. 2.3 (1) Let $0<r<R$ and take $r^{\prime}=r / R$. Then by Lemma 2.1-(1)

$$
\theta^{(p)}(X) \ll \frac{c p !}{(1-X)^{p+1}} \ll \frac{C\left(r^{\prime}\right)}{r^{\prime p}} \theta^{(p)}\left(\frac{X}{r^{\prime}}\right),
$$

hence

$$
\frac{1}{R^{p}} \theta^{(p)}\left(\frac{X}{R}\right) \ll \frac{1}{R^{p}} \frac{c p !}{\left(1-\frac{X}{R}\right)^{p+1}} \ll \frac{C\left(r^{\prime}\right)}{\left(r^{\prime} R\right)^{p}} \theta^{(p)}\left(\frac{X}{R r^{\prime}}\right)=\frac{C\left(r^{\prime}\right)}{r^{p}} \theta^{(p)}\left(\frac{X}{r}\right)
$$

and the first inequality in (2.7) holds. It also holds that

$$
p ! \theta(X) \ll \frac{c p !}{(1-X)} \ll \frac{c p !}{(1-X)^{p+1}} \ll \frac{C\left(r^{\prime}\right)}{r^{\prime p}} \theta^{(p)}\left(\frac{X}{r^{\prime}}\right) .
$$

Hence

$$
\frac{p !}{R^{p}} \theta\left(\frac{X}{R}\right) \ll \frac{C\left(r^{\prime}\right)}{\left(r^{\prime} R\right)^{p}} \theta^{(p)}\left(X / R r^{\prime}\right)=\frac{C\left(r^{\prime}\right)}{r^{p}} \theta^{(p)}(X / r)
$$

and we have the second inequality.

(2) It follows from (2.4)

$$
\begin{aligned}
& \Theta_{0}^{\left(\ell_{1}\right)}(R ; X) \Theta_{0}^{\left(\ell_{2}\right)}(R ; X)=R^{-\left(\ell_{1}+\ell_{2}\right)} \theta^{\left(\ell_{1}\right)}\left(\frac{X}{R}\right) \theta^{\left(\ell_{2}\right)}\left(\frac{X}{R}\right) \\
& \ll R^{-\left(\ell_{1}+\ell_{2}\right)} \theta^{\left(\ell_{2}\right)}\left(\frac{X}{R}\right) \ll R^{-\ell_{1}} \Theta_{0}^{\left(\ell_{2}\right)}(R ; X) .
\end{aligned}
$$

(3) It follows from Lemma 2.1 that $(p+1) \theta^{(p)}(X / R) / R^{p} \ll C R \theta^{(p+1)}(X / R) /$ $R^{p+1}$, which implies $(p+1) \Theta_{0}^{(p)}(R ; X) \ll C R \Theta_{0}^{(p+1)}(R ; X)$ and (2.9) follows.

(4) Let $0<R<R^{\prime}$. Then $\Theta_{0}^{(p)}\left(R^{\prime} ; X\right)=R^{\prime-p} \theta^{(p)}\left(X / R^{\prime}\right) \ll R^{\prime-p} \theta^{(s)}(X / R) \ll$ $\left(R / R^{\prime}\right)^{p} R^{-p} \theta^{(p)}(X / R)=\left(R / R^{\prime}\right)^{p} \Theta_{0}^{(p)}(R ; X)$.

(5) Estimate $\Theta_{0}(X / R) \ll c\left(1-\frac{X}{R}\right)^{-1}$ holds. Differentiating it $p$-times, we have $\Theta_{0}^{(p)}(X / R) \ll \frac{c p !}{R^{p}\left(1-\frac{X}{R}\right)^{-p-1}},\left|\Theta_{0}^{(p)}(X / R)\right| \leq C 2^{p} p ! / R^{p}$ and $(2.12)$, if $|X| \leq$ $R / 2$.

Proof of Proposition 2.4. (1) From (2.8) we have $\overbrace{\Theta_{0}^{(l)}(R ; X) \cdots \Theta_{0}^{(l)}(R ; X)}^{r}$ $\ll \Theta_{0}^{(l)}(R ; X) / R^{(r-1) l}$ and differentiate $n$-times, we have $(2.13)$.

(2) By Lemma 2.2

$$
\sum_{\left\{\begin{array}{c}
\left(q_{1}, q_{2}, \cdots, q_{r}\right) \in \mathbb{N}^{r} \\
q_{1}+q_{2}+\cdots+q_{r}=q
\end{array}\right.} \prod_{\substack{r \\
i=1}}^{r} \frac{\theta^{\left(p_{i}+q_{i}+\ell_{i}\right)}(X / R)}{R^{p_{i}+q_{i}+\ell_{i}} q_{i} !} \ll \frac{\theta^{\left(p+q+\ell^{\prime}\right)}(X / R)}{R^{p+q+\sum_{i=1}^{r} \ell_{i}} q !} .
$$


Hence

$$
\sum_{\left\{\begin{array}{c}
\left(q_{1}, q_{2}, \cdots, q_{r}\right) \in \mathbb{N}^{r} \\
q_{1}+q_{2}+\cdots+q_{r}=q
\end{array}\right.} \prod_{i=1}^{r} \Theta_{-q_{i}}^{\left(p_{i}+q_{i}+\ell_{i}\right)}(R ; X) \ll \frac{1}{R^{\sum_{i=1}^{r} \ell_{i}-\ell^{\prime}}} \Theta_{-q}^{\left(p+q+\ell^{\prime}\right)}(R ; X) .
$$

By $R^{-\left(\sum_{i=1}^{r} \ell_{i}-\ell^{\prime}\right)} \leq R^{-\ell^{\prime}(r-1)}$ for $0<R<1$ we have $(2.14)$.

(3) By Proposition 2.3-(3) we choose $R>0$ so small that $\Theta_{0}^{(p+l)}(R ; X) \ll$ $\Theta_{0}^{(p+l+1)}(R ; X) / 2(p+1)$. Since $s>0$ or $k \geq 1,\{n \in \mathbb{N} ; p=[s n]+k n\} \subset$ $\left\{\frac{p}{s+k} \leq n<\frac{p+1}{s+k}\right\}$, hence $\#\{n \in \mathbb{N} \mid p=[s n]+k n\} \leq \frac{1}{s+k}+1$, which is used in the following. We have

$$
\begin{aligned}
& \sum_{\substack{n_{1}, n_{2} \cdots, n_{r} \in \mathbb{N} \\
n_{1}+n_{2}+\cdots+n_{r}=n}} \frac{\Theta_{0}^{\left(\left[s n_{1}\right]+k n_{1}+\ell\right)}(R ; X) \cdots \Theta_{0}^{\left(\left[s n_{r}\right]+k n_{r}+\ell\right)}(R ; X)}{\left(\left[s n_{1}\right]+k n_{1}\right) ! \cdots\left(\left[s n_{r}\right]+k n_{r}\right) !} \\
& \ll C^{\prime} \sum_{\left\{\begin{array}{l}
p_{1}, p_{2} \cdots, p_{r} \in \mathbb{N} \\
p_{1}+p_{2}+\cdots+p_{r} \leq[s n]+k n
\end{array}\right\}} \frac{\Theta_{0}^{\left(p_{1}+\ell\right)}(R ; X) \Theta_{0}^{\left(p_{2}+\ell\right)}(R ; X) \cdots \Theta_{0}^{\left(p_{r}+\ell\right)}(R ; X)}{p_{1} ! p_{2} ! \cdots p_{r} !} \\
& \ll \frac{C^{\prime}}{R^{\ell(r-1)}} \sum_{p=0}^{[s n]+k n} \frac{\Theta_{0}^{(p+\ell)}(R ; X)}{p !} \\
& \ll \frac{C^{\prime}}{R^{\ell(r-1)}}\left(\sum_{p=0}^{[s n]+k n}\left(\frac{1}{2}\right)^{[s n]+k n-p}\right) \frac{\Theta_{0}^{([s n]+k n+\ell)}(R ; X)}{([s n]+k n) !} \\
& \ll \frac{2 C^{\prime}}{R^{\ell(r-1)}} \frac{\Theta_{0}^{([s n]+k n+\ell)}(R ; X)}{([s n]+k n) !},
\end{aligned}
$$

hence

$$
\sum_{\left\{\begin{array}{c}
\left(n_{1}, n_{2} \cdots, n_{r}\right) \in \mathbb{N}^{r} \\
n_{1}+n_{2}+\cdots+n_{r}=n
\end{array}\right\}} \prod_{i=1}^{r} \Theta_{-\left[s n_{i}\right]-k n_{i}}^{\left(\left[s n_{i}\right]+k n_{i}+\ell\right)}(R ; X) \ll \frac{C}{R^{\ell(r-1)}} \Theta_{-[s n]-k n}^{([s n]+k n+\ell)}(R ; X) .
$$

We also have

$$
\begin{aligned}
& \left.([s n]+k n) ! \sum_{\substack{n_{1}, n_{2} \cdots, n_{r} \in \mathbb{N} \\
n_{1}+n_{2}+\cdots+n_{r}=n}}\right\} \\
\ll \frac{2}{R^{\ell(r-1)}} \Theta_{0}^{([s n]+k n+\ell)}(R ; X) . & \frac{\Theta_{0}^{\left(\left[s n_{1}\right]+k n_{1}+\ell\right)}(R ; X) \cdots \Theta_{0}^{\left(\left[s n_{r}\right]+k n_{r}+\ell\right)}(R ; X)}{\left(\left[s n_{1}\right]+k n_{1}\right) ! \cdots\left(\left[s n_{r}\right]+k n_{r}\right) !}
\end{aligned}
$$

Since $\frac{(k n) !}{\left(k n_{1}\right) ! \cdots\left(k n_{r}\right) !} \leq \frac{([s n]+k n) !}{\left(\left[s n_{1}\right]+k n_{1}\right) ! \cdots\left(\left[s n_{r}\right]+k n_{r}\right) !}$, we have the second inequality in (2.15). 
(4) It follows from (2.7) that there is a constant $C=C(r /(R-\delta))$ such that

$$
\begin{aligned}
& \sum_{i=s}^{+\infty} \delta^{i-s} \Theta_{-i}^{(i)}(R ; X) \ll c \sum_{i=s}^{+\infty} \frac{\delta^{i-s}}{R^{i}} \frac{1}{\left(1-\frac{X}{R}\right)^{i+1}} \\
& =\frac{c}{R^{s}} \frac{1}{\left(1-\frac{X}{R}\right)^{s+1}} \sum_{i=0}^{+\infty} \frac{\delta^{i}}{R^{i}} \frac{1}{\left(1-\frac{X}{R}\right)^{i}}=\frac{c}{R^{s-1}} \frac{1}{\left(1-\frac{X}{R}\right)^{s}} \frac{1}{(R-\delta)\left(1-\frac{X}{R-\delta}\right)} \\
& \ll \frac{c}{(R-\delta)^{s}} \frac{1}{\left(1-\frac{X}{R-\delta}\right)^{s+1}} \ll C \Theta_{-s}^{(s)}(r ; X) .
\end{aligned}
$$

Next let us give the proof of Propositions 2.5 and 2.7 and Corollary 2.6 concering estimates of functions on sectorial regions. Let us remember notations. Let $S=\left\{t \in \mathbb{C} ; 0<|t|<T^{*},|\arg t|<\theta^{*}\right\}, 0<T_{0}<T_{1}<T^{*}$ and $0<\theta_{0}<\theta_{1}<\theta^{*}$ with $\theta_{1}-\theta_{0}<\pi / 6$. For $0 \leq \tau \leq 1$

$$
S^{\tau}=\left\{t \in \mathbb{C} ; 0<|t|<(1-\tau) T_{0}+\tau T_{1},|\arg t|<(1-\tau) \theta_{0}+\tau \theta_{1}\right\} .
$$

Proof of Proposition 2.5. (1) Let $\tau^{\prime}=\tau+(1-\tau) /(q+1), \varepsilon_{0}=\min \left\{\theta_{1}-\right.$ $\left.\theta_{0}, \frac{T_{1}-T_{0}}{|t|}\right\}$ and $\varepsilon=\left(\tau^{\prime}-\tau\right) \varepsilon_{0}$. Suppose $t \in S^{\tau}$ and $|\zeta-t| \leq(\sin \varepsilon)|t|$. Then we have $|\arg \zeta-\arg t| \leq \varepsilon \leq\left(\theta_{1}-\theta_{0}\right)\left(\tau^{\prime}-\tau\right)$ and $|\zeta-t| \leq|t| \varepsilon \leq\left(T_{1}-T_{0}\right)\left(\tau^{\prime}-\tau\right)$. Hence $|\arg \zeta| \leq|\arg t|+|\arg \zeta-\arg t|<(1-\tau) \theta_{0}+\tau \theta_{1}+\left(\theta_{1}-\theta_{0}\right)\left(\tau^{\prime}-\tau\right)=$ $\left(1-\tau^{\prime}\right) \theta_{0}+\tau^{\prime} \theta_{1}$ and $|\zeta| \leq|t|+|\zeta-t|<(1-\tau) T_{0}+\tau T_{1}+\left(T_{1}-T_{0}\right)\left(\tau^{\prime}-\tau\right)=$ $\left(1-\tau^{\prime}\right) T_{0}+\tau^{\prime} T_{1}$. Thus $\{\zeta \in \mathbb{C} ;|\zeta-t| \leq(\sin \varepsilon)|t|\} \subset S^{\tau^{\prime}}$ for $t \in S^{\tau}$. Suppose $q>0$. Then $\tau<\tau^{\prime}<1$ and we have

$$
\begin{aligned}
\left|t f^{\prime}(t)\right| & \leq \frac{1}{2 \pi} \int_{|\zeta-t|=(\sin \varepsilon)|t|} \frac{|t f(\zeta)|}{|\zeta-t|^{2}}|d \zeta| \leq \frac{C_{1} M((1+\varepsilon)|t|)^{s}}{\left(1-\tau^{\prime}\right)^{q} \varepsilon} \\
& \leq \frac{C_{1} M e^{\varepsilon s}|t|^{s}}{\left(1-\tau^{\prime}\right)^{q} \varepsilon}=\frac{C_{1} M e^{c\left(\tau^{\prime}-\tau\right) s}}{\left(1-\tau^{\prime}\right)^{q}\left(\tau^{\prime}-\tau\right) \varepsilon_{0}}|t|^{s} .
\end{aligned}
$$

Since

$$
\frac{e^{c\left(\tau^{\prime}-\tau\right) s}}{\left(1-\tau^{\prime}\right)^{q}\left(\tau^{\prime}-\tau\right)} \leq \frac{(q+1) e^{c s /(q+1)}}{(1-\tau)^{q+1}}\left(1+\frac{1}{q}\right)^{q} \leq \frac{(q+1) e^{c s /(q+1)}}{(1-\tau)^{q+1}} e,
$$

we have (2.18). Suppose $q=0$. Then $\tau^{\prime}=1$ and $|f(t)| \leq M|t|^{s}$ for $t \in S^{1}$ and we have

$$
\begin{aligned}
\left|t f^{\prime}(t)\right| & \leq \frac{1}{2 \pi} \int_{|\zeta-t|=(\sin \varepsilon)|t|} \frac{|t f(\zeta)|}{|\zeta-t|^{2}}|d \zeta| \\
& \leq \frac{(1+\varepsilon)^{s}}{\varepsilon} C_{1} M|t|^{s} \leq \frac{e^{\varepsilon s}}{(1-\tau)} C_{2} M|t|^{s}
\end{aligned}
$$


(2) Let $\tau^{\prime}=\tau+(1-\tau) /(q+1), \varepsilon=\left(\tau^{\prime}-\tau\right)\left(\theta_{1}-\theta_{0}\right)$ and $\rho=\min \left\{(\sin \varepsilon)|t|, t_{1}-t_{0}\right\}$. Let $t \in S^{\tau}$ with $|t| \leq t_{0}$ and $|\zeta-t| \leq \rho$. Then we have $|\arg \zeta| \leq\left(1-\tau^{\prime}\right) \theta_{0}+\tau^{\prime} \theta_{1}$ and $|\zeta| \leq|t|+|\zeta-t| \leq t_{0}+\rho \leq t_{1}$. Thus $\{\zeta \in \mathbb{C} ;|\zeta-t| \leq \rho\} \subset S^{\tau^{\prime}} \cap\left\{|\zeta| \leq t_{1}\right\}$. Suppose $q>0$. Then $\tau<\tau^{\prime}<1$ and we have by $\rho \leq(\sin \varepsilon)|t| \leq \varepsilon|t|$ and the assumption on $f(t)$,

$$
\begin{aligned}
\left|t^{n} f^{(n)}(t)\right| & \leq \frac{n !}{2 \pi} \int_{|\zeta-t|=\rho} \frac{\left|t^{n} f(\zeta)\right|}{|\zeta-t|^{n+1}}|d \zeta| \leq \frac{M n !|t|^{n}((1+\varepsilon)|t|)^{s}}{\left(1-\tau^{\prime}\right)^{q} \rho^{n}} \\
& \leq \frac{M n ! e^{\frac{c s}{q+1}}|t|^{s+n}}{\left(1-\tau^{\prime}\right)^{q} \rho^{n}}
\end{aligned}
$$

If $\rho=(\sin \varepsilon)|t|$, then

$$
\frac{|t|^{s+n}}{\left(1-\tau^{\prime}\right)^{q} \rho^{n}} \leq \frac{C_{1}^{n}|t|^{s}}{\left(1-\tau^{\prime}\right)^{q}\left(\tau^{\prime}-\tau\right)^{n}} \leq \frac{\left(C_{1}(q+1)\right)^{n} e|t|^{s}}{(1-\tau)^{q+n}} .
$$

If $\rho=t_{1}-t_{0}$, then for $|t| \leq t_{0}$

$$
\frac{|t|^{s+n}}{\left(1-\tau^{\prime}\right)^{q} \rho^{n}} \leq \frac{e|t|^{s+n}}{(1-\tau)^{q}\left(t_{1}-t_{0}\right)^{n}} \leq \frac{e|t|^{s}}{(1-\tau)^{q}}\left(\frac{t_{0}}{t_{1}-t_{0}}\right)^{n},
$$

and we have the estimate. Suppose $q=0$. Then $\tau^{\prime}=1$ and $|f(t)| \leq M|t|^{s}$ for $t \in S^{1}$ and we have

$$
\begin{aligned}
\left|t^{n} f^{(n)}(t)\right| & \leq \frac{n !}{2 \pi} \int_{|\zeta-t|=\rho} \frac{\left|t^{n} f(\zeta)\right|}{|\zeta-t|^{n+1}}|d \zeta| \leq \frac{M n !|t|^{n}}{\rho^{n}}((1+\varepsilon)|t|)^{s} \\
& \leq \frac{M n ! e^{c s}|t|^{s+n}}{\rho^{n}}
\end{aligned}
$$

and the estimate (2.20) by the same way as $q>0$.

Corollary 2.6 easily follows from Proposition 2.5.

Proof of Proposition 2.7. Take $0<\tau_{0}<1$ such that $T \Subset S^{\tau_{0}}$. Then it follows from Corollary 2.6 that there is a constant $C_{1}$ such that

$$
\vartheta^{\alpha_{0}(i)} \partial^{\alpha^{\prime}(i)} u_{i}(t, x) \underset{x^{*}}{\ll} \frac{C_{0} C_{1}^{s_{i}+1}}{\left(1-\tau_{0}\right)^{\alpha_{0}(i)}}|t|^{s_{i}} \Theta^{\left(\left|\alpha^{\prime}(i)\right|\right)}\left(r ; X-X^{*}\right) .
$$

Since $|\alpha(i)| \leq m$, there is a constant $C_{2}$ such that

$$
\prod_{i=1}^{\ell} \vartheta^{\alpha_{0}(i)} \partial^{\alpha^{\prime}(i)} u_{i}(t, x) \underset{x^{*}}{\ll} \frac{C_{2}^{\ell+s}}{\left(1-\tau_{0}\right)^{m \ell}}|t|^{s} \Theta^{(m)}\left(r ; X-X^{*}\right) .
$$

Hence from the assumption on $b(t, x)$

$$
b(t, x) \prod_{i=1}^{\ell} \vartheta^{\alpha_{0}(i)} \partial^{\alpha^{\prime}(i)} u_{i}(t, x) \underset{x^{*}}{\ll} \frac{B C_{3} C_{2}^{\ell+s}}{\left(1-\tau_{0}\right)^{m \ell}}|t|^{s} \Theta^{(m)}\left(r ; X-X^{*}\right)
$$

and we have (2.22) for some constant $C$ depending on $\tau_{0}$. 


\section{References}

[1] R. Gérard and H. Tahara, Singular nonlinear partial differential equations, Aspects of Mathematics, Friedr, Vieweg \& Sohn, Braunschweig, 1996. viii+269 pp.

[2] P. D. Lax, Nonlinear hyperbolic equations, Comm. Pure Appl. Math. 6 (1953), 231-258.

[3] T. Mandai, The method of Frobenius to Fuchsian partial differential equations, J. Math. Soc. Japan 52 (2000), no. 3, 645-672.

[4] S. Öuchi, Genuine solutions and formal solutions with Gevrey type estimates of nonlinear partial differential equations, J. Math. Sci. Univ. Tokyo 2 (1995), no. 2, 375-417.

[5] Singular solutions with asymptotic expansion of linear partial differential equations in the complex domain, Publ. Res. Inst. Math. Sci. 34 (1998), no. 4, 291-311.

[6] - Growth property and slowly increasing behaviour of singular solutions of linear partial differential equations in the complex domain, J. Math. Soc. Japan 52 (2000), no. 4, 767-792.

[7] Asymptotic expansion of singular solutions and the characteristic polygon of linear partial differential equations in the complex domain, Publ. Res. Inst. Math. Sci. 36 (2000), no. 4, 457-482.

[8] The behaviors of singular solutions of partial differential equations in some class in the complex domain, in Partial differential equations and mathematical physics (Tokyo, 2001), 177-194, Birkhäuser, Boston, Boston, MA.

[9] Multisummability of formal power series solutions of nonlinear partial differential equations in complex domains, Asymptot. Anal. 47 (2006), no. 3-4, 187-225

[10] complex domain, Algebraic Analysis of Differential Equations -from Microlocal Analysis to Exponential Asymptotics- held at RIMS Kyoto Univ. in 2005.

[11] H. Tahara and H. Yamazawa, Structure of solutions of nonlinear partial differential equations of Gérard-Tahara type, Publ. Res. Inst. Math. Sci. 41 (2005), no. 2, 339-373.

[12] C. Wagschal, Problème de Cauchy analytique, à données méromorphes, J. Math. Pures Appl. (9) $\mathbf{5 1}$ (1972), 375-397. 